$3 x$

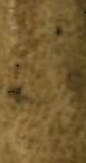 \\ .92

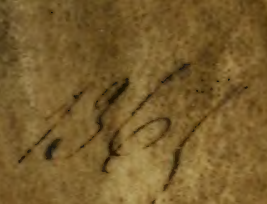 \\ Sis +2
}

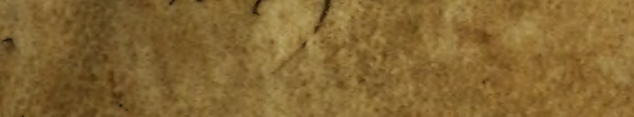

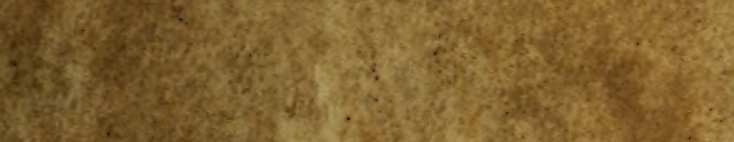

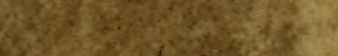

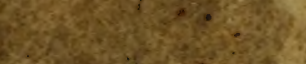

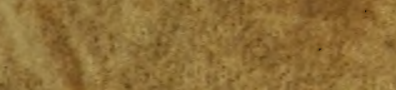

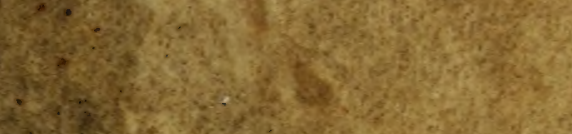

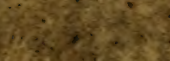

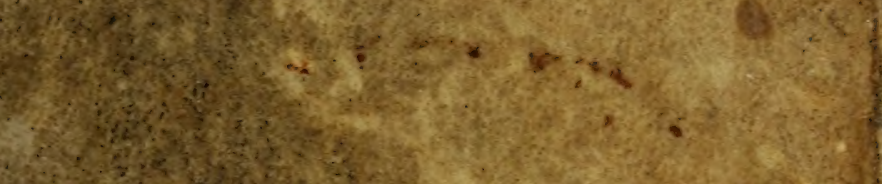

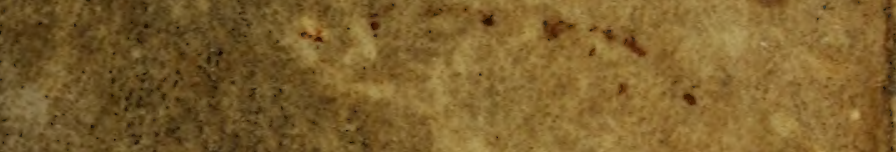

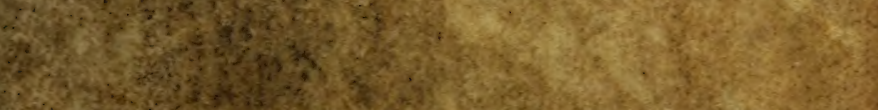

20.6.

(2)

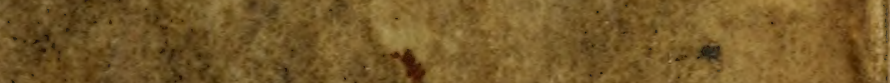

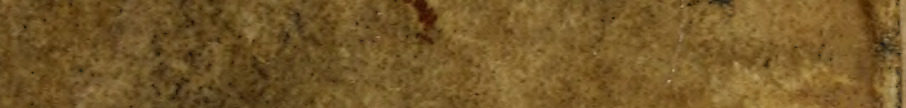

23.

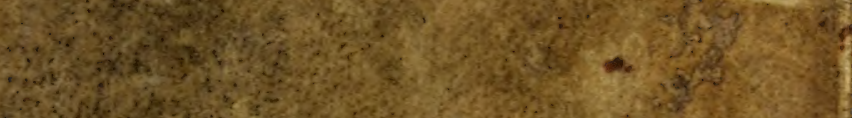

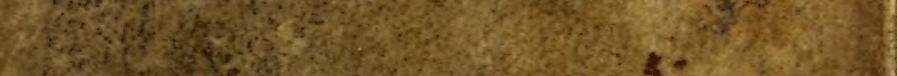
Shing

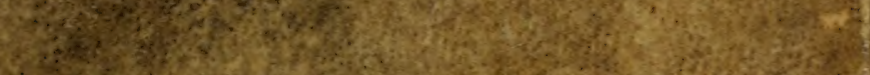

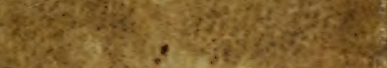

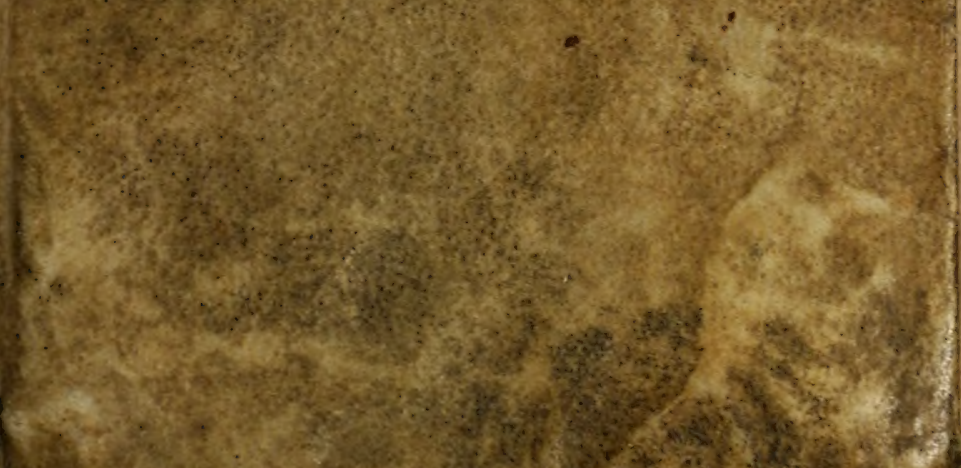


in 3 a

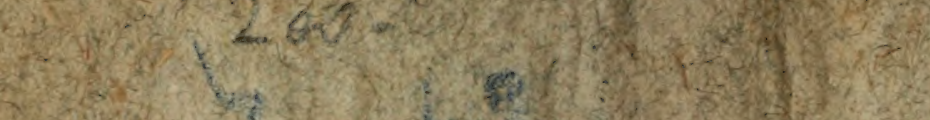

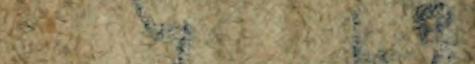

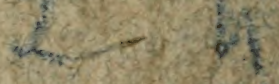

3020,298

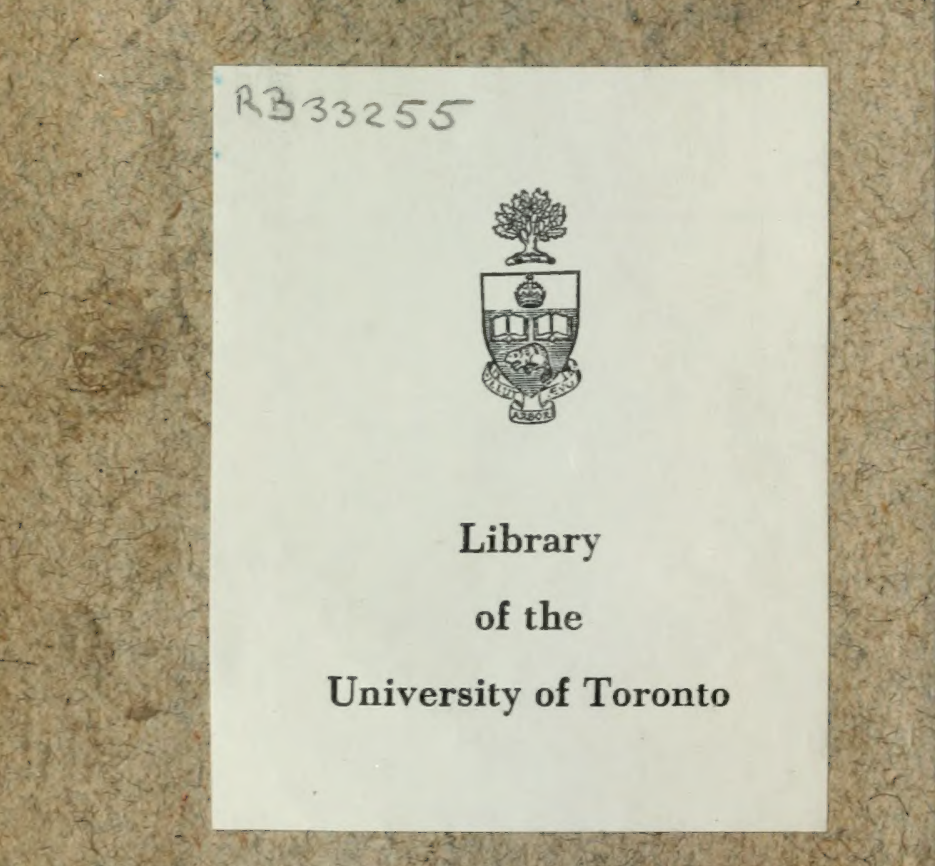

Le:

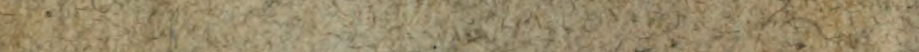

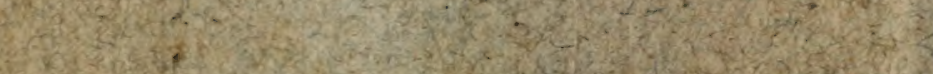

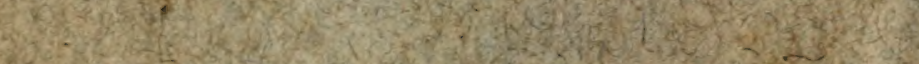

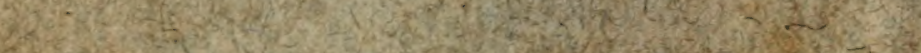

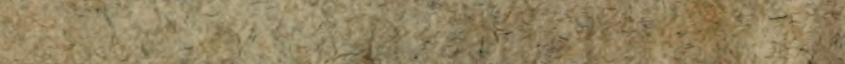

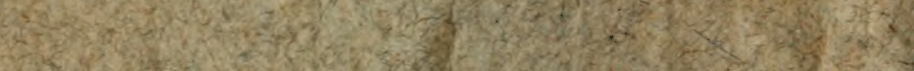

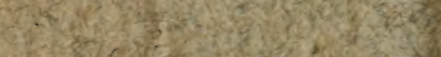
15
$y$

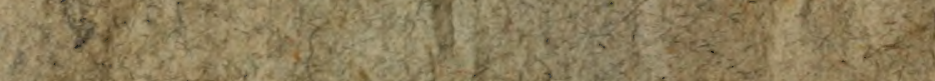
(3) 
$+\quad L E$

IE R

FRANCOIS culciuer les Arbres, \& I terbes Potageres: Atrec la maniere de conferuer les Fruirs, \& faire toutes fortes de Conficures, Conferues, \& Maflepans.

DEDIE AVX DAMES.

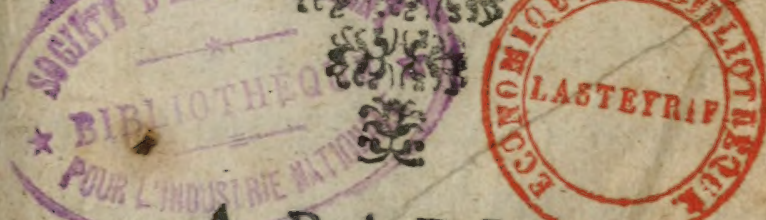

\section{A PARIS,}

Chez IACeV Es LAISNE', fur le Quay, \& pres les Auguftins à la fleur de Iys. 

हो है (ris

E P I T R E

\section{A V X}
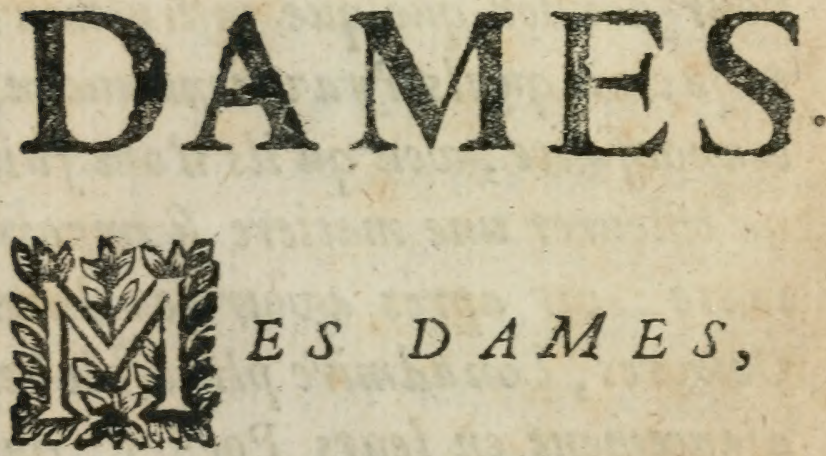

ES DAMES,

Ie n'ay pas defein de faire icy on Panegyrique de la beauté, vtilité, er necefjité des Iardins, its parlent aßsez d'eus-smefmes pour fe fuire efimer: joint auffi que beaucoup de braves Hommes, tanc Anciens, que de nofire temps, fe 


\section{E PISTRE}

font pleus à en defcrire les excellences, छs ont employé les plus belles beures de leurs loifirs pour en exalter les beautez, tant en Vers, qu'en Profe: mais la plufpart fe font Servis du Jujet pluttoft pour faire paroiftre la force de leur Gonie, que pour donner quelque inftruction; Ej jecrois qu'ils n'y avoient aucune connoiffance, vou qu'ils n'ont fait queffleurer une matiere fi importante: car aprés avoir leu leurs Oeurres, l'on admire plûtoft le bel ajancement en leurs Poëfies, que lon ne tive inftruction de ce quils ont écrit : refervé Monfieur de la Serre, qui a composé le Theatre d'Agriculture en Pro $\int e$, arvecuntel ordre, que lon le lit Es relit Jans aucun dégouft: mais comme il a rraitté generaleme nt de tout le mé- 


\section{$A V X$ D A M ES.}

nage de la Cariagne il n’a pû par. ticularifer ce qui eft neceffaire pour le Iardinage, qui eft le feul fujet que je pretens vous faire voir en ce Livre. Il porte pour tiltre LE IARDINIER FRANçOIS, à caufe que tous ceux quit on ont traitte riont parle que de leur ciimats. par exemple, Monfieur dela Serre, a compose fon Oeurere en Languedoc, qui of un Pays bieit dirjeren de celuy de Paris E' de Jes environs, pour lequel $j$ ay deffein dicire. comme ayant fait experience en ce Climat de tont ce que je diray: je le dedie Avx DAMEs, mais particulierement auxmenageres, d'au tant que cbacune a fes inclinations particulicres; les unes fe pla ifents aux charmans accords de la Mufi. que; les autres en la lecture des 


\section{EPITRE}

Romans e.) belles pieces en Frofe, n'en laiffant efchapper aucunes quielles ne lewr donnent rang Jur la Tablette; les autres à la Commedie, er aux doux accens des vers: les autres affectionnent les Tableaux des bons Maiftres, les autres font Cabinet de toutes fortes de curioficez, et) les autres eiment pafjonnement les bonnes odeurs, छ? Gomet journies de quantitề d'effences, Es poudresprecieufes; ainfi diuerfement chacune fu it fon inclination, (toutes lourables en leur's curiofi. tez; ) mais comme elles ne les rechercbent que pour fatisfaire les Sens, celles que iay nommées n'en regardent chacune qu'on feul: le I ardin à sette preroagatiue par deffus les autres, quil donne dequo\} Les Jatis, aire touscinq : l'odorat, 


\section{$A V X D A M E S$.}

trousue fon contentement dans la quantité des fleurs, \&o des fruits qui portent odeurs: la veuë fe rejoüit en la diuerfité des couleurs qui s'y rencontrent, fiviues que tous les plus excellens Peintres demeurent court en imitant leur beautez, (O) les couleurs les plus fines, font ter. nies par leur efclat: pour le Gouft, il $\int u f f i t$ de dire, que les frians $\varepsilon^{\circ}$ delicats apres s'efire plus que fuffufamment gorgez de plufeurs fortes de bons mets, n'eftiment pas auoir fait bonne chere s'ils ne finifent leurs feftins par les fruits : qui fans eftre affaifonnez que de la Na. ture, fe trouuent neantmoins fi excollens, chacun en fon espece, qu'il. fuut auoüer que les fruits feuls emportent le prix en la atisfaction du Gouft : l Ouic qui femble en eftre 


\section{EPISTRE}

exiblfe, me fait dire pourtant qu' it niy a point d: contentement qui ofgule celesy d'entendre louer la beaut3 devojure lardin, (t) particulie rement la grofeur (t) diuerfité de ros frits: le Tact y troune aufs for plaifir en les maniant (4) pe. Lint: aucuns de fquels ont la peau $\sqrt{b}$ selicate, quelle a befoin d'vne ming fubtile (t) legere, pour la leuer auec plus de pröprieté. Outre cis contentemens, iy en remarque encore trois tres-abantageux: Le piemier E le plus loüable eft celuy des prefens que vous en faites à pernunes de toutes fortes de condition, $\int_{\text {dont vous eftes amplement remer. }}$ ciêes @o loüies dans voftrecuriofte: le lecond, que voftre maifon en eft fournie, Es le troifiefme (qui vous fera aimer voftre menage- 


\section{$A V X D A M E S$.}

ment) c'eft que vons pourrez vcrzdre la lewé des fruits d'vne quan. tité de vos Arbres à des fruitiers, qui vous payeront argent comptant @o paraduance, furquoy vous fouderez one partie de oofore reue. nu. Ie connois des Dames de grande condition qui en $v$ fent de la for. te, @o auowent que ce projit leus fait affectionner dauantage lewp din, El les rend plus liberales aux defpenfes neceflaires qu'il $y$ conuient faire. C eft pourquoy (MES D AMES ] iefpere que vous me Sçaurez gré, de vous auoir dreß $\hat{e}^{A}$ cette inf Iruction, ie l'ay fait impri. msr en petit volume, afin que vous le puifficz porter fans incommodi. té, pour confronter le trauail de oos lardinicers auec ce petit liure, e) inger de leur copacitc ou neglis 


\section{$E P I S T R E$}

gence. I'aymis à lafin du traité des

Fruits, vn Catalogue tres-ample de tous leurs noms : quand il ne feruiroit que pour vous en entretenir dans les Compagnies, encore en pourrez wous retirer quelque agreable Satisfaction. Et pour comble du ferrice que ie defore vous rendre, ie conclus par la maniere de conferast vos fruits, tant en leur naturel, que fechez: Or par wne grande facilité de faire toutes fortes de Cinfitures, Paftes, Gelées, Conferues, Ca Majlepans, Jans s'embaraffer beaucoup, vious fuppliant d'excufer mes defauts par tont out rous les rencontrerez, e. de neme pascondamner, quiapres que vous aurez experimenté le contraire de ce que iefaris par les of. preunes que vous en ferez: Faites- 


\section{$A V X D$ A IIES.}

moy aujJ la grace de croire, que ie ne me ferois bazardé de mettre ce petit Oeurure en lumiere, $f i$ ien'a. vois fait les experiences de tout ce quily eft compris. Agrécz donc [ s'il wous plaift] ce petit trawail, qui wous eft offert auec tant de foumiflon, Par

\section{$M E S$ DAMES,}

Toftre tres-obeylfant feruiceur, R.D. C. D. V.V.B, D. N. 
$P R E F A C E$.

\section{au Lecteur.}

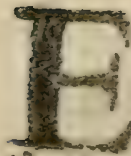

iole bien me

Nirez dás celardin qui que vous foysz, Cher Lecieur, bien ne prometre que vous y trouncrez voltre fatisfa. ction: Si vous eftes fçauant au Iardinage, vous verrez la belle dipolition quieftanstoute fon enceince, ranc en nos Arbres, qu'en nos Herbages, manurang que vons anez allez de courtoi. fie pour approuuer noltre perie Labeur; il n'eft pas dreffé pour vous inltruire, car ie fuppole que vous cn fçauez beaucoup plus que moy, \& m'eftimerois treshourcux ( $f_{1}$ i auois lhonneur de 


\section{Preface an Lederer.}

voftre connoiffance) que vousme voulufiez fouffrir a upres de vous pour en tirer des Leçons: cen'elt que pour ceux qui n'y ont aucune connoiffance, ou bien petite, que i'ay voulu efcrire; par riculierement pour infture tane de pautures gens qui trouueroiét leurs iournées, sils fçauoient vn peu fe demefler du Iardin; ie me fuis rendu le plus intelligible qu'il m'a efté polfible dans les rermes les plus communs de no. ftre Langue; fi iay vfé fouuent de redites, c'eft afin qu'ils com. priffent auec plus de facilité ce que ie defre qu'ils f̧̧achent, ce qui leur eft exrémement neceffaire; ils le pourrontappren. dre dans les deux premiers trairez. Car le troifiefme ce n'e? 


\section{$A V$ LECTEVR,}

pas pour le dehors, il n'eft que pour le dedans du logis. Les Sommeliers des grandes maifons auouẻront quilil ne leur eft pas entierement inutile, pour (dans l'embarras qu'ils ont quelque. fois) fe reflowuenir de plufieurs gentilleffes que le chágemēt des faifons leur pourroit auoir faic oublier à caufe du long-temps qu'ily peut auoir, qu'ils ne les ont prariquées. Les perfonnes de qualié peuuent fe diuertiren la lecture de noltre Liure; car cóme ils prennent plaifir à leurs lardinages, ils pourrone s'en entretenirauec leur Iardiniers, \& leur commander fort à propos ce qu'ils defurent quils executent. Les Bourgeois qui ont des Maifons de plaifir proche de Paris 


\section{AV IECTEVR.}

\& qui neveulent faire la defpenfe d'y entretenir vn lardinier, peuuent donner vn de ces liures a leurs Vignerons, qui caufera qua ces fortes de gens (quoy que grofliers) ne laifferont pas defefaçonner; cequi fera qu'ils en feront mieux feruis, \& leur Iardin mieux entretenu. Brefic ne vois perfonne qui puiffe refufer la promenade de noltre Iardin, \& à qui elle ne foit neceffaire pour fon contentement \& pour l'ceconomie de fes prouifions. Ilyadmirera la bonté de l'Autheur de la Nature, qui a voulu obliger l'Homme (quoy qu'ingrat) à reconnoiftre la mul. ritude de fes bienfaits, en l'in. nombrable diuerfité des Arbres, \& Plances, qui contentent la 


\section{AV LECTEVR:}

veue par leurs beauté, \& fatis: font à l'entretien de la vie, non feulement pour la neceffité,mais encore iufques à l'excez: Ie le prie quil vous donne les faifons propres à embellir voltre lardin, \&fauorife toutes vos entreprifes. 
*.

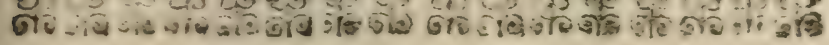

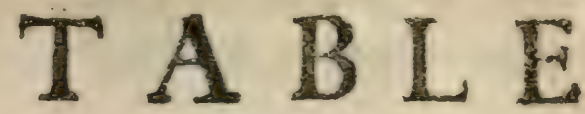

DES PRINCIPAVX Sujers de ce Liure.

\section{PREMIER TRAITE',}

SECTION PRFMIERE.

TI Lieu, de la T erre è fond du I ardin, 1 auec le moyen de metre en valeur lo wejchant Terre.

\section{SECTION II.}

Des Efpaliers, Contr' E paliers, $\sim$ Buiffors. \& $S E C T I O N$ III.

Des Arbres, \& du choix gue lion en deie faire.

SEC T I O N IV.

16

Dela Pepiniere or Bapardiere.

\section{$S E C T I O N$ V.}

Des Greffes, or ce qui eft a ob eruer pour les shoibutomner.

$$
\text { SECTION VI. }
$$

De lo mamiere de Greffer. 


\section{T A B L E. \\ SECTION VII.}

Des Arbres, of Arbuftes en parrioulier, de de leur gounernement of remedes à leurs - maladees.

so

\section{CATALOGVE DES NOMS} Des Fruits dont nous avons la connoiflance au climat de Paris.

Poires defguelles le E ruit eft en $\int a$ maturis tédan's la fin du mais de I uin o luillet. 72. En Iniliet, fo Sitt. ideme En Aorfo, Co Septembre. En Seprembre, or Octebre. Fn Oitobre, i Noversabre. En Novembre, of Decembre.

En Decembre, Ianvier. 80 En Ianvier, of Fevriep, $8 \mathrm{I}$ En Fevrier, co autres mois juinans iusques aux nounelles.

Pommiers hatif: Pommes de garde.

Pruniers batifs si tardif:

28.

83

85

87

Pejchers \& Panies.

89 Des Cerifes, Bigarresux of smblable. 2: Des Eigues. 


\section{T A. B. L.E.}

Des Orenges, Cutrons, critres Arbies aliviellix.

\section{SECOND TRAITE}

SECTION PREMIERE。

Des Melvers, Concombres, Citroüilles. of autres ejpeces remblables.
SEC I ION II.

93

Des Antichaser, Cardonser Aprorges. I0?:

SECTION III.

DesChoux $\rightarrow$ der Lectuês de to stes elyeces. II 6 SEC.TION IV.

Des Ravines.

$$
\text { SEC TION V. }
$$

De toutes les fortes d'Herbes Potageres. I 47

$$
\text { SECTION VI. }
$$

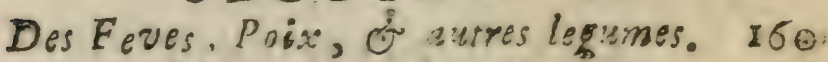

$$
\text { SECTION VII. }
$$

Des Oignons, Ails, Cibonles, Poirreauxs. Herbes $O$ dariferenies, \& autres commodite, du Iardin; quine font comprifes ainx Clazz, pitres precedens, 


\section{T A B L E}

III. ET DERNIER TRAITE'.

SECTION PREMIERE.

De la maniere de conferuer les Frusiss en leup naturel.

Des fruits fecher.

SECTION II.

\section{SECTION III.}

200

Des Conjurures an Sel, of ina igre. 206 SECTION I V.

Des Confurires an Mouf ou an $F$ in doux an Cidre, of all Miel. $211 \mathrm{II}$ SECTION $V$.

Des Confinres liquides an Sucre.

$$
\text { SEC TION VI. }
$$

268

Des Confurures au fec, or Pastes de fruits 243

SECTION VII.

Des Conjerues or Aaliepains. 261 . 


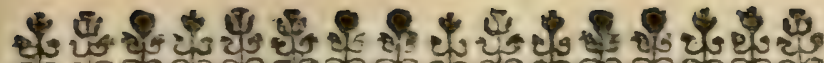

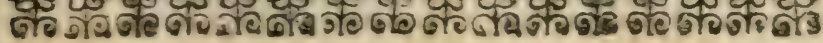

\section{E}

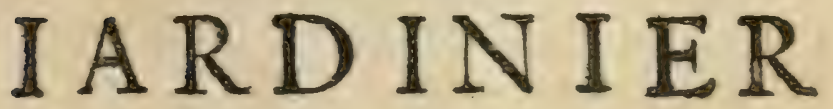

FRA N C O S

s

PREMIER TRAITTE'

Dis lieu, de la $T$ erre \& F Fond du Iardie auec le mogen de mettre en valeur la mefchante Terre.

SECTION PREMIERE。

7 Ov s ceux qui ont écrit du ménage de la Campagne; ont ap-

1. porté des fujections fi grandes en la difpofition des Baftimens, \& autres parties du Domaine, qu'il eft du tout impolfible de pousoir dreffer vn Lieur comme ils vous le prefcriuent; d'autant que les fituations ne s'accordent pas toutes a leur fouhait: $C^{\prime}$ eft pourquoy ie ne vous veux affujettir en aucune faço:a 


\section{LE IAKDINIEK}

à placer voltre Iardin, vous vous feruirezdes lieux comme vous les trouuerez, s'ils font defia faits, ou bien ( auec bon confeil, vous en drefferez un notueau, au lieu qui conuicndrale mieux à voftre Baftiment.

Quant à la Terre fi vous la rencoutrez bonne, ce vous fera yn grand aduantage \& vne grande efpargne, mais rarenient en trouluerez-vous oû il n'y wit beaucoup à tramailler : car telle Terre fera bonne ald delfus, quir eftant ouuerte de la hauteiz d'un fer de Befche feulensent) fe trouuera Arguilleufe deflous, quieft vn fonds pire aux Arbres que le Tuf; parce que dans le Tuf ils peuuent irouler quelques petites $\mathrm{Yei}$ nes qui donneront paffage aux $R$ acines pourchercher la fraicheur plus bas, \& prendre quelque nourriture;mais la $T$ erreArgilleule, ou Frarche, (quieft vne Terre auec laquelle les Boulangers de Paris font los Atres de leurs Fours, ) eft comme vn Plancher, øui par fa dureté \& d'enfité ne peut eftre percé par les Racines, \& dans les grandes ardeurs de lEfté, empefche que la fraicheur de delfous ne puiffe penetrer à trauers, ce gui caufe que les Arbres \& autresPlantes font tellement delfechez, qu'au lieu 


\section{FrA NçoIs.}

d'zuancer en leur accroilfement, ils ne font que languir, \& periffent enfin.

Pour remedier â ce deffaut, il n'y a qu'un feul moyen, quieft d'effondrer \& rompre cette Terre de trois à quatre pieds d: profondeur, commençant par vne Tranchée large de quatre à cinq pieds, de toute la longueur de ce que vous voulez faire effondrer, en ievtant les Terres touies d'un colté : \& voltre Tranchée eftant vuidée, \& curèe iufqu'a la profondeur que vous defirerez, vous mettrez au fonds des longsFumiers, du Marc de Preffoir, à Vin ou d Cidre, de la Fougere, fi vous en auez la comruodicé, qui eft le meilleur de tous les Fumiers, des fueilles d'Arbres, iufques mefmes à de mefchantes A mes de Fagot, de la Mouffe, \& autres chofe femblables: bref, ce quivous fera le plus facile \& a moindre defpenfe : car toute l'inuention que l'on peut auoir en effondrant, n'eft que fourtenir la i erre creule, afin que la Fraic eur de deffous puiffe reuigauter les Arbres \& Plantes, durant les exceffiues chaleurs.

Vous en mettrez enuiron drmy ritd de haut au foids de voftre Travichise. apresquoy vous en feresvale bes. In be paresllelargaur. 
4 LF IA DINIIR

re deffus qui eft la meilleure fur ces Fumiers, \& creufant iufques a pareille profonde ur que la premiere, vous remplirez voltre premiere Tranchée, \& la Terre de deffous fe trouuera deffus, continuant à faire de femblables Tranchées, iufques à la fin de ce que vous voudrez faire effondrer.

Vous me pourrez objecter que cette Terre qu'aurez tirée de deffous ferz infertile: I'advouë auec vous que la premiere année elle ne ferz paroiftre $\{\mathrm{a}$ bonté : mais quand par les pluyes \& gelées d'un Hyuer, clle zura efté meurie ( auec le peu d'amendement que l'on metrra ) elle produira bien plus abondamment que ne faifoit celle de deffus, qui eftant vfée par le long-temps qu'ily a qu'elle porte, a perdu vne grande par. tie de fa vertu.

Toutes fortes de Temps ne font pas propres à ce trauail ; car durant les grandesChaleurs, cette Terre eft tellement dure \& fcellée, que les Pics, \& pioches $n^{\prime}$ y peutuent du tout entrer : $1^{\prime}$ Hyuer y eft plus propre quaucune autre Saifon, d'autant que lespluyes d'Autonne ayant humecte la Terre, la rendront plus facile à eitre befchée ou foüillée, \& a aufí qu'en ce temps-là il Pleut, Neige, ou Geleal- 


\section{F. R A $M$ çoIs.}

fez fouuent, ce qui aide beaucoup à cet ceuure : ioint aufif que les payfans n'eftans pas alors beaucoup occupez, fe donnent à meilleur marché qu'és autres Temps qu'en on trauaille aux Vignes, ou durant l'Aouft? aufquels Temps l'on ne les peut auoir qu'a peine \& à force d'Argent.

Pource qui eit du fonds où fe rencontre le Tuf, vous le mefnagerez, comnous venons de dire, en le faifant rompre, \& les pierres eftant fur le Gueret feront emportées hors du Iardin. Si le Tuf n'eft guere e fpois, \& quaseftant rompu vous trousuez du Sablon, ou eutre petit Tuf mouuant, il fuffira de le rompre , ne le tirez pas hors de la Tranchée; car les Arbres ietteront affez de Racines dans ce petit Tuf, à caufe des elgoutures du Fumier qui fera deffus.

Vous obferuerez qua fonds de la Tranchée d'où l'on aura tiré du Tuf, vous ne mettrez que de bon fumier demy confomme, d'autant que l'Eau des pluyes, ou des arroufemens pafferoit facilementa trauers, fi c'eftoit des Marcs de Preffeur de la fougere, ou autres que i’ay nommez cy-deuant.

I'apprehende que vous ne me difiez. que s'eft s'embarquer en vne grande defo 
penfe, que de faire effondrer enticrement vn Iardin : Ie l'a ouë auffi auee Tous mais c'elt nne fois pour iamais, \& le profit que l'on tire d'vn tel trauail, recompenfe au centuple les frais que vous y aurez faits : à caufe que les Arbres en feront plus beaux n'eftans ny mouffus, ny galleux, \& qu'ils en portent des fruits plus gros fans comparaition, que ecuxqui font plante $z$ en une Terre qui n'eft pas effondrée; les Artichaux, Foirées, \& autres Legumes, y viennent monttrueufement gros, bref vous ferez-in fatisfait voyant la difference de ce que produifoit voftre Iardin auant qu'il fuft effondré, à ce qu'il produira parapres, que vous noaurez aucun regret à vo re defpenfe.

Si vous voulez pourtant eftre plus re. tenu, ie vous enfeigneray vn autre moyen d'amander voltre Iardin autec moins de fraits; mais auffi comme la defpenfe fera moindre, ce qu'il produira n'en viendra pas fi beau; i'en traitteray cy-apres au plantage des Efpalicrs, \&r au Potage.

Il y a beaucoup de Curieux qui s'étentent encore au dela : car ils font paffer la Terre par vne Claye, pour en ofter tontes les Pierres: ce qui le fait en polant 


\section{F R N C O Is.}

cette Claye, ou Cribbe, fur le bord de la Tranchée, \& iettant de paëllées de Terre fur le haut du Crible, la Terre paffe \& les Pierres roulent au pied du Crible, puis on les emporte hors du Iardin.

La forme de ce Crible eft vn Chaflis de menuiferie de deux grands poulces d'efpois, de fix pieds de haut, \& de cinq de large, lequel aura deux trauerfes dans fa hauteur, de la mefme groffeur du Chaflis, \& toutes les quatre pieces trauerfantes feront percées également de la groffeur d'une Baguetre, defquels les Chandeliers de Paris fe feruent pour façonner leur Chandelle, diftans l'vn de lautre d'un trauers de doigt, dans lefquels vous mettrez des Baguettes de Couldre, s'il le peut : car c'eft le bois le plus ferme \& durquand il eft fec, \& qui refifte le pluslong-temps fans fe rompre quaucun autre. Il faut que le haut \& le bas de voftre Chaffis foient percez à iour, afin que quand il y aura des Baguettes rompuës, vous en puifiez reinettre facilement d'autres, les arreftant auec des petits Coins par les bouts. 
Des Efpaliers, Contr'Efpaliers, Buifjonse

\section{SECTION II.}

Es Elpaliers eftans le principal orne$L$ ment des lardins, il eft raifonna. ble de leur donner le premier lieu, \& en efcrire amplement : ce fera auffi le fuiet où ie $m^{2}$ arrefteray le plus dans ce premier Traité.

Par l'Espalier sous entendons parler des Arbres, dont les Murs des Iardins font parez : pour le mettre en eftat il faut faire vne belle Tranchée, comme i a dit cy-deuant, fi le fond eft d'Argille vous vous gouluernerez com meàl'A rgile: sil eft de Tufcomme au Tuf, vous laiffe. rez vn pied de terre fans rompre proche du Múur, de crainte de $l^{2}$ endommages, Fuis ayant $m$ is vn lict de fumier de demy pied de haut, ou fond de voftre Tranchée, fous ferez ietter deffus ce fumier, la hauteur d'un pied de la meilleur $\mathrm{T}$ errs qứarez tirée de voftre Tranchée : ce: la fait vous marquerez les places, our volks voudrez planter vos Arbres, qui fera à vne diftance raifonnable: celle de douze pieds me femble la plus conuenz- 
bie : toutefois à voltre difcretion, ie ne vous donneray point la Loy, chacun ayant fon opinion particuliere: mais mon raifonnement eft, que s'ils font plus pres, ils fenuiront dans peu dannées : fi plus loing \& qu'il vienne à mourir va Arbre, ou qu'il en faluft greffer quelqu'autre, dont le frait ne vous plairoit $\mathrm{p}$ as, vous feriez ennuyè de voir $\mathrm{fi}$ long-temps voftre Muraille defcouuerte en cet endroit-là.

\section{Ayant marqué la place de vos Arbres,} fuiuant la mefure de douze pieds, vous ferez remplir la place où vous les planterez (trois pieds de chaque colté de voftre marque) de la meilleure Terre, que vous meflerez auec du petit Fumier de vieille Couche de Melons, ou autre qui aura feruy à efleuer les Herbages de voftre Iardin, \& il vous reftera vn efpace de fix pieds, dans laquelle vous ferez mettre vn fecond lit de fumier de $\mathrm{Va}$ che, Porc, ou Moutons bien confommé \&2 biengras: puis rous ietterez deffus le refte de la Terre qu'aurez tirée de voftre Tranchée, \& drelferez voftre Gueṛet, le rendant bien uny.

Vous ferez faire des trous pour vos Arbres, aux endroits qua aurez marqué, as les planterez bien proprement, farians 
vne petite butte dans le milieu du trou, afin qu'ayant pofé voftre Arbe deflus, vous puiffiez eftendre les $R$ acines autour de cette petite butte, les tirant el bas: puis le trou eftant remply, \& la Terre dreffée, vous la marcherés tout autour de l'Arbre pour l'alleurer \& empefcher que la Terre ne fe trouue creufe en quelque endroit.

Vous pourrez (fivous voulez) aunat que de planter, abbatre la Terre iufques au Mur, vn pied de chaque coltéde l'endroit où vous voulez placer vós arbres, fans crainte d'endommager voftro Mur.

Vous les placerez à vn pied prés diz Mur, un peuen penchant les branches contre le Mur, afin de leur donner la grace en leur accroiffement, cela fera aufir que les Racines feront plus dans le milieu de voltre tranchée, pour y chereher leur nourriture auec plus de facilité.

Prenez bien garde à ne mettre autre fumier prés les racines de vos arbres, que de ce menu de vieille Couche, encore faudra-il le menter aliec beaucoup de bonne Terre; car l'E?é bruneroičtout : d'autant que le grand Fumier tient toufiours la Terre creufe, iufques à ce qu'il foit confommé : $S i$ ie vous en fais mettre dedans les efpaces, $\epsilon^{\prime}$ eft que vos 


\section{FRA ÇOIS.}

Arbres ayant pristerre, \& leurs Racines allane la deux ou troifiefme année cher. cher la bonté de ce Fumier, qui fera confommé, ils poufferont de plus beaux Bois, \& produiront de tres-beaux \& tres-gros fruits.

Pour ce qui eft de dreffer vos Efpaliers, ie vous veux enfeigner plufieurs façons deles accommoder, felon l'aage de vos arbres.

La premiere, ce fera de ficher de petits pieux en Terre, àdemy pied de vo. ftre Mur, pour commencer à conduire les tendres jets que vos arbres poulfe1 ront, \& s'il eft befoin d'y adioufter quelquestrauerfes quel'on appelle Lattes, vous y en pourrez mettre ce qui fera necellaire, liant vos icunes jets auce de petits Oziers, aujoncs, fanslesferrer, mais feulement pour les conduire.

La feconde maniere fera de faire vne Haye de Pieur, \& Lattes maillez également, \& bien liez, qui ayant plus de force que la premiere, obligera les arbres à prendre tel ply que l'on voudra.

La troifiefme eft vn Treillifiage attaché au Mur, \& fupporté par des Os de jambe de Cheuaux, ou par des Crochets de Fert, fcellez dans le Mur, à caufe que l'arbre montant \& s'enforciffant, fe 
ietter oir tout en dehors, pour chercher l'Air, \& romproit ou feroit verfer la Haye, dont les Pieux font fichez dans la Terre qui fe laboure, \& outre ce qu'á la longueur du temps ils fe pourriffent.

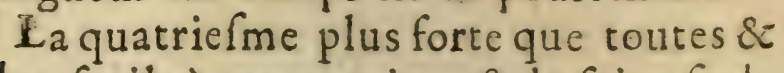
plus facile à entretenir, eft de faire fceller dans le Mur des bouts de bois de la grolfeur d'un fort Chevron, qui font equarris à huit Pans ègaux, \& fortiront le Mur de fix poulces feulement, aufquels ferez faire des trous de Tartiere d'va poulce $\&$ demy de profondeur, à deux poulces prés du bout, vous le ferez fceller à égale diftance, de hauteur \& largeur : au milieu de chaque quarré en fee ra encore fcellé vn; faifant la figure d'vr. Franc de Quarreau. Vous prendrez des. Efchalats que vous aurez fait faire de longueur de la diftance que vos bouts de Cheurons feront placez, lefquels vous affilerez par les deux bouts pour entrer dans les trous qui feront au bout des Chevrons, \& pour les y placer, vous les plierez va peu en forme d'Arc, pofant les deux bouts dans les trous qui feront *is à vis les vins des autres, \& laiffant aller l'Arc, ils tiendront d'eux mefmes fans aucune lieure : la figure qui eft aus commencement de ce iraité yous le monfte affez clairement. 


\section{FRA N çOIs}

Quand vos arbres feront defia forts ils n'auront befoin d'eftre conduits auec tant de bois qu' en leur ieunelfe, il fuffira d'en arrefter les plus fortes branches à cette forte d'Efpalier: Et quand ily aura quelqu'vn de ces Efchalats qui fera pourry, l'on en remettra facilement vn autre, en ayant toufiours de prouifion dans la maifon.

La cinquiefme, de prendre du bois de quartier vn peu plus gros qu'vn Efcha. lats, le dreifer des crochets de fer, ou des os deCheuaux, comme i'ay dit cydeuant, \& le lier auec du fil d'archail, ou de cuivre, cela dure extremement longtemps.

La fixiefme \&derniere façond.Efpaliet les arbres (qui eft la plus belle \& la plus agreable : mais ellere re peut facilement faire quaux Murs en plaftre) c'eft de prendre de petites Lanieres de cuir, ou lizieres de drap, anec lefquelles vous arrefterez les ieunes branches, attachant ces Lizieres au Mur auec du cloud, \& les branches prendront leur ply en grofliffant, ne pouuant l'arbre fe ierter en a. vant, ny arracher le cloud qui fe rouilile dansle Mur.

Ces trois dernieres façons d'Efpalier ie pratiquent pour bannir les limats, 
14 I. IA RDINIER

perce-oreilles, marcinets \& autres in. fectes qui fe meticut dans le lien des mailles, \& entre leseficorces du bois qui eft rond, \& non pas de quartier.

Vous obleruerez de ne planter aucun arbre dans les coins, ou angles de vos Murs: pourcequ'ils ne pourrcient prendre que demie nourriture, \& que cela arondiroit la figure de voltre Iardin, \& aufi que l'arbre ietteroit cout fon bois en deuant pour auoir de l'air.

Le Contr'Efpalier eft vne Haye qui forme toutes les allies du Iardin: Il ie plantera de mefme maniere que l'Elpalier referué que la Iranc: ée fera de quatre pieds de large, au moins; \& que vous ferez jetter vos Terres, la bonne d'vi colté, \& la moindre de l'autre, zfinde mettre la bonne ali Fonds de voftre Fof$f^{\prime}$, \& la moindre delfus.

Vous y planterez vos arbres tous droits, \& non panchez comme a l'Elpalier.

Le boisquiles foutiendra, il faut de necefficé qu'il foit fiché dans la Terre, \& maillé de Latces; toute la propricté \& curiofité quis’y peut apporter, eft de le faire de bois de quartier, \& le lier suec dufil de Fer, ou de Cuiure.

Quelques vns pour efpargner la defpente de l'entretien dubois, fe conten. 
tent quand les arbres font forts, de les lailfer ioindre enfemble, \& les lier, mais il faut qu'ils ayent efté plantez à neufpieds l'yn de l'autre, \& le mal eft cu'ils font fuiets à eftre ébranlez des vents.

Les Builfons, ce font arbres que l'on plante ordinairement dans les bandes des Parteres, \& dans les quarer des Potrgers, le long des Sentiers : lefquels on tailie de telle figure que l'on veut, ou ronds, ou quarrez, ou plats pardelfus, ou que l'on laille monter en forme de Cyprez: En les taillant on fe contente pluitolt de leur donner la forme, que d'elpargner les bourgeons à fruit, que l'on comferueroit aux Efpaliers, \& Cuntr'Efpaliers.

Vous les planterez aux lieux les plus conuenables de vos bandes \& à égale diftance l'vne de l'autre, obleruant ce que íay dit en la façon de planter.

La maniere que ie vous viens de donner pour planter vos arbres, vous elpargnera 12 defuenfe de faire effondrer cout volte Iardin, les Allées n'en ayans pas beaucoup de befoin : car auant que les arbres ayent poullé leurs Racines iufques aux allées, ils auront pris force; \& les perceront aifement où ils 
I6 LE IARDINIER

trouueront la bonne Terre. Vous ne laifferez pourtant vos allées en friche, ains les ferez nettoyer d'herbes, \& fur tout ofter le Chiendent iufques au dernier brin, renuerfant la Terre de la hauteur d'vn fer de Befche; en fecoüant le Chienden : \& $f$ apres ce labour il repouffoit quelque peu, vous le ferez chercher profondement enraciné qu'il foit, pour le bannir entierement de voftre Iardin comme tres-nuifible.

Des cribres, of dis choix que l'on en doit faire.

\section{SECTION III.}

En'elt rien fait d'auoir bien prepare voftre Terre, fi vous ne la plantez de beaux \& bonsarbres, que vous pourrez choifrir dans les Pepinieres de gens qui font en repuation e' eftre fidels: car la plufpart de ceux qui en vendent, trompent foutent les acheteurs. A ceux-cy àe ne vous confeille den prendre aucun, que vous ne royez le fruit deflus, 2 les retenir dés ce temps-là en les cachetane auec de petites bandes de Parchemin, fellées de voftre Cacher, pour en les leuant eitre alfuáé d'aueir ce qu'aurez 
achete' : à ceux-là qui liurent fidelle. ment, yous ne ferez pas fiexact, il eft bon pourtant de les cacheter auffi, quand ce ne feroit que pour faire voir à ceux qui en acheteront apres vous que ce font arbres retenus.

Si vous youlez remarquer les efpeces, vous le pourrez en deux façons, l'vne en pendant de petites ardoifes, cù le nom de l'arbre fera en efcrit, \& l'autre en y mettant des bouts de Laine teinte de plufieurs couleurs, dont vous ferez vn memoire; cela feruira à difcerner vos arbres en les plantant, afin que diftinguant ceux d'Efté d'auec ceux d'Hyuer, yos Efpaliers, Contr'Efpaliers \& Buiffons en foient plus agreables à voir, n'eftans pas denuez entieremene par endroits, où il n'y auroit que des fruits d'Efte, $\&$ aufil qque vous n'en mettrez point deux d'vne mefme forte proches $l^{2} \nabla n$ de l'autre.

Les fruits que vous choifirez, particulierement quant aux Poires) fi vous en youlez vendre, ce fera Bon Creftien d'Efté \& d'Hyuer, le Mufcat haftif gros \& petit, le Portail, la Bergamotte d'E. fté \& d'Hyuer, faint Lezin, Adamotte, Bezidairy, Double fleur, gros Roulfelet de Rheims, parfum, Bœuré des deux 
I8 LE IARDINIER

fortes, Meflire Iean de Cire, Cadillac: $\&$ autres que vois fçaurez qui fe vendent le plus cher.

Pour les Pommiers, la Reinctte de plufieurs fortes, le (orr-pendu, Caluil-Rouge dedans, Chaftaigner, Apis gros \& petit, Pigeonnet ou de Judée, \& autres.

Autant aux Pefches \& Abricots ils fe vendent toufiours bien, mais ces eleux fortes de fruits ne font gueres propresen Efpalier, à caufe que les branches meurent tantcft d'vn bras, tantoft d'vn autre, \& bien foument cntierement : ce qui eft fort defagreable à voir fur les brefches que cela fait à vos Efpaliers : Celle que l'on eftime le plus font les AuantPefches, ou Pefches de Troye Alberges, Pauies, Pefches, Cerifes, Violettes, de Pau, Brignons \& autres.

Pour les Cerifes \& Bigarreaux, d'autant qu'il s'en fait des plants particuliers, ie $n$ en feray autre mention, finonde dire que celles à Courte-querës \& à petit noyau, pareilles à colle de la Vallée de Montmorency font les plus excellentes.

11 y a les Precoces qui fe plantent à grand abry \& belafpcet du Midy, ou ie metrent dans des Quailfes, pour eftre ferrées pendant l'Hyuer auec les Orcu- 


\section{FrA nçoIs.}

gers, mais c'elt plutioul pour la curionice gue pour le profit

Recournons à l'Election de nos arbres, Si quecetre digreffion ne nous elloigne point d'en dire tout ce qu'il le pourra, particulierement des Poiriers, comme portant le fruit le plus precieux de vo. ftre Iardin.

L'Arbre greffé fur la Coignalle en à preferer à cout autre, d'autant qu'il rapporie pluftolt \& fait le fruit plus gros, \& plus beau, eltant rouge du colté duSoleil, \& jaune de l'autre colté qui ết ombragé par fon efpoitfeur.

Ceux fur Franc font eftimez porter des fruits de meilleur gouft, mais ils ne viennent if gros, ny libcaux en couleur que cenx qui lurst greffez fur la Coignarle, qui elt ce que nous recherchons pour lavente, d'autant que les autres Poires reftent touliours vertes en couleur.

Pour l'âge que deuez choifir vos arbres, quatre ans ou enuiron eft la plus belle grandeur, d'autant que plus ieunes ils ne garniroient pas voftre Efpalier promptement : \& plus vieils ils auront ietté de groffes Racines, que l'on rompra, ou efclattera en les replantant au grand preiudice de l'Arbre, qui fera long-temps à recoururir la playe, $2 x$ fau- 


\section{LEIARDYNIER}

dra qu'il iette beaucoup de nouluea $C$ Che. uelu, auant que de bien faire.

$L^{\prime}$ opinion de beaucoupeft, qu'il n'e?t que de planter gros, \& qu $^{\circ} \mathrm{vn}$ arbre eft zrop long-temps à venir : ie ne fuis pas de leur aduis, car i’eftime que vn arbre bienchoifi, \& de belle venuë, de l'âge que iay dit, iettera de plus beau bois, qu'vn autre plus vicil, qui ne pouffera que de petit bois quoy qu' en quantité.

Pour la forme, vous prendrez garde qu'ils ne foient point Mouflus ny Afrouchis, mais de belle venuë, le bois gros \& bienclair, que $l^{2} E$ fculfon ou la Fente ayent bien recouvert leur Saunageau. qu'il foit garny dés le bas pour etre plus agreable en Efpalier.

Vous les ferez leuer en woltre prefence. afin que l'on rompe le moins qu'il fe pourra de cheuelu, $2 x$ que l'on n'efclate ny coupe alacune racine s'il fe peut.

Choifffez vn beau iour enuiron la faint Martin : cardepuis que la fueille com. mence à tontber il n'y a aucun peril à leuer des arbres: vous les ferez emporter le plus doucement qu'il fe pourra, fur le dosdes Hommes, ou fur des beftes Afi. nes, \& les planterez le pluftoft qu'il vous fera poffible; de peur qu'ils ne $f_{a}$ tiquent, \& que le petit cheuelu ne fe def- 


\section{F R A N çoIs.}

feiche: en les plantant vous couperez le petit bout des racines, pour ofter feulement ce qui pourroit eftre deffeiché.

Vous ne les taillerez qu'en la faifor', pour les caufes que ie vous diraycy-apres.

Aux Poiriers Greffez fur franc, illeure faut couper le piuot: afin que les autres Racines prennent force, \& s'eftendent tout autour en cherchant la meilleuro Terre.

Les alteres arbres de toutes fortes feront arrachez, replantez, \& gouuernez de la melme façon que les Poiriers, n'y syant aucune diftinotion aे faire en cet endroit.

Quanta la Taille des Arbres, le vray temps pour ceux qui font vieils plantez, elt dans le decours de la Lune de Ianvier, qui eft aufil le temps que l'on ctreille des Greffes pour la Fente \& Couronne. Et pour ceux qui font nouveaus replantez, ils ne feront taillez qu'alors que la Séue commence à monter, afin que la playe fe recouure pluftoft : car fi pous les taillez dés l'Hyuer, le bois fe delfeichera par lesgelées a l'endroit de la taille, \& $\mathrm{fe-}$ roit vn Argot de bois mort iufgues à l'cil qui poufferoit le plus proche de la taille.

Pour ce qui eft de lier, \& dreffer les 
Arbres, le mois de Fevrier y eft plus propre, d'autant que les plus grandes geléeseltant paflées, l'on ne feint point de couper le fuperflus, \& auffi que la Séue n'eftant pas encore montée, l'on cft hors de danger d'ébourgeonner les yeux qui font noïez à fruit.

La principale fuicetion de bien dieffer des arbres, eft de les eftendre en forme d'eruentail outere : ceft à dire que comme les baftons d'vn efuentail ne fe croifent point lesvizs fur les autres, aufli les branches de vos arbres feront conduites de meime façon.

La plufpart des Iardiniers du commun tombent dans cet erreur de les croifer, à caufe que l'on ne les a iamais repris, s'émancipans de vouloir gouuerner les arbres, qui eft vne connoilfance toute particulicre, qui ne s'apprend pas chez les planteurs de Chour; ils font encore des fautes notables, c'eft qu'ils fagottent vne quantité de menuès branches routes dans vn lieu, ce qui ne fe peut fouffrir fans les en reprimander : car l'on ne doit laiffer quel'épaiffeur d'vne branche par tout larbre : \& pour comble de leur ignorance, ils paffent \& repaffent les branches autour du bois que l'on a mis pour les dreffer, on mettent 
l'Arbre derriere, \& le bois en deuant, qui font des fautes fi grandes, quelles ne fe peuuent palfer fans leur reprocher leur incapacite : Ie les pris charitablement de donner vne année de leur temps au feruice de quelque bon Iardinier, ou ils pourront apprendre à gouuerner les arbres, \& proficer de ces corrections. $S$ 'il fe rencontroit pourtant quelque place à l'arbre qui ne fut pas garnie, vous poutuez en cettenecefítè-là croifer quelque petice branche pour couurir ce vuide, mais que ce foit rarement, \& faites en forte quel'on ne s'en puilfe pas aifement apperceuoir.

Il eft necelfaire de donner quatre labours d̀ ros arbres par chacun an, \& vous pourrezfaire femer furces labours de perites herbes qui fe leueront d'vn labour a l'autre, comme Laictuë, Pourpier, Cerfueil, chicorée, melme y efleuer de ieunes Choux pour replanter, brefrout cequi fe leue, \& ne feiourne pas longtempsen vn endroit : vous y pourrezauffi replanter des Laictuës pour pommer, de la Chicoréc pour blanchir; \& du Pourpier pour confre au Sel, \& pour la Graine, cela vous portera double profit, car vos arbres outre le Labour feront srroufez par le Iardinier, qui aura foin 


\section{I I IARDINIER}

d'efleuer ces petits Herbages.

Vous ne mettrez pres de vos Arbres aucune Racine telle qu'elle foit, tant a caufe qu'elles veulent feiourner longcemps dans Terre pour leur aceroiffement, qu'à caufe qu'elles emmaigriffent, ou effritent beoucoup la Terre.

Les Choux à Pommes, autres grands Choux, \& les Poirées poutr tirer des Cardes, en ferontauffi bannis.

Il fera neceffaire aux arbres vieils plan. rez, de trøis en trois, ou de quatre en quatre ans, de les defchauffer, \& als bout des $R$ acines hors de la Terre qui ef en Labour, faire vne Tranchée ar y mettre de bon fumier, vous laifferez pourtant quelque peu de Terre fur les Racines, de crainte que le hâle de l'Efté ne les brufle : le vray temps pour cet cusure eft al commencement de l'Hyuer, dalstant que le fumier fera demy confommé auant les chaleurs.

De la Popiniere, ef Baffardiere. SECTION IV.

T A Pepiniere eftant le commencement de l'efleuement des Arbres, il oft neceffaire de vous donner l'inftru-

ation 


$$
\text { FI A N çO I } S \text {. }
$$

ivion enciere de quelle maniere clle veur cltie gounernée. C'ent pourquoy nous commencerons par les Semences

I orates Senences defirent vn lieu frais, mon efrouffe darbres, ny remply de $\mathrm{R}$. cines: elles veulent efre abriées du Soleil de Midy par quelque Mur, vous le pourrez facilement trouuer dans voltre Iardin, cn vous feruant du lieu en labour où fera voltre Eipalier du cofté du Midy : vne année ieule fuffira pour vous foumir amplement de toute forte de Plan, '3 plusque vous n'en aurez de befoin.

Ayzant fait amas de Pepins, \& de Noyaux durant toutc vne année, à mefure que vous mangerez des fruits : \& 1 Hyuer eftant palfe, vers la fin de $\mathrm{Fe}$ vrier, vous femerez vos $P$ epins en rayon, $c^{h}$ acune efpece à part : \& vos Noyaux au Plantoir, à quatre bons doigts I'vn de l'antre, ie prefuppofe que la Terre ois vous les mestez aura efté labourće au commencement de l'Hyuer, \& qu'elle le fera tine feconde fois quand vous femerez. Les Pepins \& Noyaux poufferont dés la mefme année, qui plus forts, qui plus foibles: il n'importe, ils feront toufiours affez bons à replanter; ce n'eft pas que li vous les auiez femé dans vne 
26

\section{LE I A R I N I ER}

planche derriere voftre Contr'Efpalier, (du melmecolté du Midy, poureftreau moins abricz le Matin, \& le Soir) ilsne font meilleurs de deux ansque d'vn, pour efre replantez, mais tels qu'ils feront, vous ne laifferezd'en faire voltre Pepiniere.

Noyaux de Pefches fe plantent auffi au tempsqu’elles font en maturité, les enterrantafin que l'on cueille la Pefche de l'arbre, c'eft à dire auec fa chair, \&\& faudra metre de petits baftons à l'endroitoules aurez mis, de crainte qu'en labourant on ne rompe le Girme.

Pour commencer donc voltre Pepiniere, vous choifrez quelque partic de voltre Iardin que ferez labourer, \& bien dreller: par apres vous la ferez marcher pour affermir la Terre, puisferez faire de petites Rigolesde la hauteur, \& largeur du fer d"vne "efche, diftanies de deux pieds \& demy l'vn de l'autre, iettant laT erre toute d'v n cofté fur le bord du rayon : cela fait vous poferez voftre Plandans le rayon, l'appuyant de l'autre cofté que vous aurez mis la Terre qu'aurez tirée, \& luy ayant auparavant rognéle Piuot, vous ne le mettrez qu’à demy pied l'vn de l'autre, chaque efpece à part, les poiriersauec les poiricrs, les 


\section{FRA $A$ \& I S.}

pommiers auec les pommicrs, \& ainf des auires : puis vous remplirez le rayon de fa Terre, \& marcherez delfus pour 1 affermir, de crainte que le plan ne s'éuente, \& ferez foigneux que l'Herbe n'eltouffe le Plan, le faifani labourer, \& farcler, quand il en aura befoin.

Vous ne rognerez vo re I lan que quand la Séue voudra monier, \& le fe.. rez ébourgeonner iufques à vin pied de haut, afin qu'il ne fe troule point de nœuds en l'efcorce, qui vous nuilfent quand il las faudra greffer.

Sidés l'annee merme que vous les aurez plantez il s'en trovinoit daflez forts pour efculfonner, \& quils eulfent de la Seue, ne faites aucunes dificulté de les greffer : mon opinion elt, que l'on ne fçauroit cfculfonner les Sauuageaux \& Francs trop jeunes, pourueu que I on puille placer l'Elcullnn, il fuffit : ma raifon cit que le Sauuageau \& $10 n$ Eicuif. fon, prennent vn accroilfement égal dés leur icunefife, que la tail' e de l'Argot en eft pluftolt recouverte, \& quils poulfene deplus grande force, que ciux que l'on efcuffonne fur des plus forts, qui font des deux outrois ans à recourir la place cù on aura ofté l'Aicot, \&̈ dunt foumen. de l'autre coltéde l'Eiculion l'efcorc: d? 
2.8

$$
\text { LE IARDINIER }
$$

Saunagcais mourant de trois oll quatre doigts plus bas que l'Efcuffon, qu'il faut vn grand temps a l'arbre pour recouuris ce manque. Ioint que l'Efcorce d'vn vieil Saumagcau ne fe colle pas fi bien auec celle de l'Efculfon, \& qu'ils s'y fait vn gros bourrelet facile à decoler, ce qui ri arriue pas quand les deux Efcorces font toutes deux tendres.

Vous ferezauffi rne Pepiniere de Coignaffes, femblable à celle des Francs, quev ous gouuernerez de la mefme forte.

Les vrayes Coignaffes ( qui eft ce que ie vous nomme sauuageaux) font celles qui ont le fruit en Calle-baife; \& non pas celuy qui eft gros derriere, \& aboatit en pointe par desant.

Quant aux Pefchers que vous auez femé de Noyaux, ie fuis d'aduis que vous en faffiez vn quarré de voftre. Iardin à part, pour les caufes que ie vous ay di. res, qui font que les mettant en ESpalier, ou Contr'Efpalicr, il meurt tous les ans quelque branche, ce qui eft fort defagreable à voir. C'eft pourquoy ie vous confeille quíen l'vn des quarrez les plas efloignez du logis, ( pour ne pas empercher la veuë de tout voltre lardin ) du coftédu manuais vent, qui eft la Bize, ou Septentrion, vousy logiez vos Pefchers 


$$
\text { FRA N ÇOI } 0 \text { : }
$$

que lcuerez de voltre Seminaire, \& vous les placerezà fix pieds l'vin de l'autre, en diftances égales de rous coltez, ce qui le nomme planter è la Quinconce; \& là vous en recueillerez abondance de fruits, à cautfie de la quanticé d'arbres.

Vous ferez fuigneux de leur faire donner aufil quatre labours, faire ofter le boismort, \& couperaux deux, out troifiefme noud, les ieunes iets qui pouffarta de trop grande force, attireroient à cux fuls toute la Seue de l'arbre, \& laifferoient languir les vieilles branches, qui faute de nourriture mourroient en peu de temps, car tenez pour vne maximeque la Seue monte toufiours aux jets les plus tendres, ) vous pouvez aufti au melme lieu entremefler quelgues Abrigotiers que vous gouuernerez de la mefme façon que les Pefchers \& Pauics.

La Baftardiere fera auffi placée en quelque Carré de voltre Iardin le plus reculé du Logis, à caufe qu'elle vous cacheroit la veuë des autres carrez, paroiffant comme vn bois taillis.

Lelieu eftant deftiné, \& la Terre bien nettoyée de toutes herbes \& racines, vous ferez faire des trous tirez all cordeau de deux pieds de largecn tout fens, \& de deux autres bons pieds de profon- 
deur, difans de quatre pieds l'vn de l'au. tre, \& les rangs aunf éloignez de quatre bons pieds : vous prendrez des arbres grefiez dans voftre Pepiniere, \& les tranfplanterez dans voftre Baftardiere, il n'importe que le iet ne foit que divn an, iis ferontalfez bons à replanter : \& pour les planter vous obferuerez ponctuellement ce que $i$ ay mis deuant au plantage des Efpaliers, quieft de mener du petit fumier de vicille couche auce la bonne Terre, \& faifant vne petite butte au milieu du trou, y pofer l'arbe, eftendant les racines de tous cofiés, toufiours tirane en bas, puis apres remplir le trou iufqu’à la Greffe \& marcher la Terre pour alfeurer l'arbre.

Vous noterés qu'il faut que la Greffe foit toíjours à afleurement de terre pour l'ormement de larbre, qui feroit defagreable fil'on voyoit le nœud où il aura efté greffé \& particulierement en quel. ques-vns defquels la Greffe furpalfe le Sauuageau en grolfeur, \& faite vn gros bourrelet à la loudure de la Greffe, ce qui eft fort defagreable.

Quant aux arbres des Ffpaliers, \& Contr'Efpaliers expofés au Midy, l'on peut enterrer la Greffe de quatre doigs plus bas que le Sol, pour auoir plus de fraif- 
cheur, fans craindre quelle pouffe ancun Cheuelu à caufe de la fechereffe; \& quand mefme elle en auroit ietié, le Iardinier en befchant y peut regarder, les couper \& donner vn peu d'air au nœud, afin qu'il n'en pouffe point de nouteati.

Vous obferuerez auffi que durane les grandes Chaleurs, fi vous voulés faire beaucoup de bien à vos arbres, ce fera de mettre autour du pied ( fans pourtant toucher à larbre) de la Fougere, ou du grand Fumier, trois pieds tout autour de vos arbres, \& quatre doigts d'elpois feulement, cela feruira à ombrager \&c entretenir la fraifcheur de la Terre, \& auffi empefchera qu'eftant battuë de quelque grande pluye elle ne fe creuaf. fera, ce quréuente founent l'arbre, \& deffeiche les petites Racines, fi immediatement aulant que de meitre ce Fumier, vous faites donner vn Labourà la Terre, ce fera vn double bien que voftre arbre en receura: d'autant que elle s'entretiendra toufiours meuble, \& ne pouffcra aucunc mauuaife Herbe à trauers ce fumier.

La baftardiere vous eft neceffaire pour trois raifons principales; la premiere pour auoir des arbres de prouifion, propresà remettre en la place de ceux qui

B iiij 
meurent, on gin languiffans ne profitent point: fecondement pour dégager la confuition qui fourroit eftre dans voftre $\mathrm{Pe}-$ piniere à caufe de la trop grande quantité de ieuncs arbres : la troifiefme fera pour enanoir àvendre, en resonpenfe de la fremiere defpenfe quaurés faite à planter voltre Iardin : ils pourront auffi vous rapporter du fruit en ce lien-là dont vous tirerés grand contentement, \&toutre tuut cula, vin arbre replanté plufieurs fois efl bcaucoup plus franc, que fi diredement tiré úe la pepiniere, il eftoit placé cri licu à demeurer

Il eft auffi de befoin que vons ayez vne Baftardierc pour les Arbres greffés fur Franc, comme Poiriers, Pommiers \& autres, que vours voulés faire monter en grands Arbres do fin pieds de Tige, \& it ${ }_{13}^{3}$ y a autro gouncrucment qu' aux precedens, finon'qu'cn les plantant il faut comper le Piust, qui eft la maiftreffe Ra. cine, \&: dans leur accroilfement couper à vn petit doige pres du Trone, les Branches qui tirent trop de nourriture, \& qui feroient vin fourchon à l'Arbre, laiffant les petites, afin quele Tronc fe fortifie, en arreftant la Seue en chemin: Il y en a beaucoup quife trompent en ce rencontre, lefquels nettoyent vn Arbre de ton- 
tes fes branches, iufques à la hauteut où ils veulent que fe faffe la Tefte, \& font contraints d'y mettre vin Pieu, ou Eftançon pour le dreffer, \& le garantir des grands vents, qui font plier \& tordre le Tronc, à caufe de la charge qui eft à la tefte : cela empefche que le tronc n'en groffit pas fi tort, parce que la Seue ne faifant que paffer pour aller trouuer le nouveau, ne s'arrefte pas en chemin comme elle feroit, sil y auoit des ieunes branches

Il y a vin temps pour ébourgeonner, \& arrefter les Arbres durant la Seue : les Bourgeons que l'on peut ofter, font ceux quidans leur accroilfement donneroient quelque difformité a l'Arbre : car pour à fruir, i! les faut tous laiffer.

Pour diftinguer yn Bourgeon aे fruit, d'auec vin à bois, c'ent que celuy à bois, n’a qu'vne feuille, \& celuy à fruic en a plufieurs.

L'on taille anfi les ieunes Iets qui pouffent de trop grande force, \& qui par leur vigueur pourroient attirer tonte 13 Seue d'vn $\Lambda$ rbre, \& feroient languir les Branches qui font defia toutes venués: quand vous remarquerés cela, vous les arrefterés au deuxou troifiéme noud; ; ce aprés quoil aura pouliẻ la Selle. 
34 LE IARIINIER

On rogne auffi la Seue d'Aouft, tant parce que l'Arbre s'eftendruit trop rans re garnir, qu'à caufe que cicn foutuent elle ne meurit pas auant 1 Hyuer, \& laiffe la branche affamée par le bout, qu'il faudroit necellairement rogner à la taille de Fevrier.

Si vous voulés faire à part quelquePlan, de grands Arbres, il faut de necefficé qu'ils foient greffez fur Franc. \& non pas fur la Coignalfe quant aux Poiriers, : fur le Pommier de Paradis quant aux Pommiers : car autrement ils ne grandi roient pas, mais demeureroient toû* jours bas de tige.

Vous planterés lespomicrs à cinq thoifes au moins, \& les Poiriers, Pruniers, ¿z autres à quatre: Vous obferuerés fur tout qu'ils foient plantés à la Quinconce, $c^{3}$ eft à dire en Lignes fe coupantes à Angles droits.

L'on pourra femer dansce Plan quelques Graines, ou Legumes, cela feruira à les entretenir de Labour : car ie vous recommande fur toutes chofes de ne fouffrir aucune Herbe fauuage en tout voftre Lieu, reftraignés-vous pluftoft à vn petit, \& le menagez bien, que d'en entreprendre vn grand \& le laifferaffraichir faute de Labours: les grands lieux 


$$
\text { FRA ç C O Is. }
$$

fe font admirer, mais les petits fe cultiuent plus facilement ; \& vous tirerez plus de profit d'vin petit lieu ménagé, que d'vn qui fera negligé.

\section{Des Greffes, of ce qui est है ob irruer pour les choifur bonnes.$$
\text { SECTION V. }
$$

IL y a vne grande fujettion a bien choifir les $G$ reffes, car de là dépend que les Arbres portent promptement, ou font quelque-fois jufques à dix ou douze ans fans porter.

Les meilleures Greffes font celles qui font au bout des plus fortes \& maifreffes Branches d'vn Arbre qui a couftume de bien charger à Fruit, \& que vous voyez difpore à porter beaucoup cette année là : car de là dépend que les jeunes Arbres que vousen greffez, ont du Fruit dés la feconde où troifiéme année, par fois dés la premiere.

Comme au contraire, fi vous prenez vne Greffe fur vn jeune Arbre qui n’ait pas encore porté Fruit, celuy que vous en grefferez ne rapportera de long temps apres.

La Greffe pour l'Efcuffon, doit eftre 
36 LE I ARDINIER

cueillie dans le mois d'Aouft en decours, \& Creffée en mefme temps, pour regle plus certaine, en nes'arreftant pas tant a la Lune, c’eft quand vos Saunageaux \& Francs, font en la force delcur Seue: car l'Efculfon elt toufrours alfez bon; mais le Saurageau manque bien founent à eftre difpofé à le reccuoir faute de Seue, ce quiarriue quad l'ente eft partrop fec : quils ne pouffent point ou fort pet en la seue d'Aouft : C'eft pourquoy fi vous auez quantité d'Arbres à greffer, ne perdez point de temps, \& commencez de bonne heure.

Vous connoiftrez fi le Saunageau eft au fort de fa Seue en deux façons: I'vne en incifant l'Efcorce auec le petit Coufteau, ou Entoir, \& leuant l$^{\mathrm{P}} \mathrm{E}$ fcorce de l'Arbre, fi elle quitte le Bois, il y a de la Seue: fi elle ne le quitte point, attendez qu'elle foit montée, car vons ne le feriez que gafter: l'autre eft quand on voit au bout des Branches des Sauuageaux les Fueilles de la nouluelle Seue eftre blanches, ce qui témoigne que l'Arbre y eft.

La Greffe pour l'efculfon fera choifie du ject de l'année, bien meure, \& de belle venuë: car il y en a beaucoup qui font aigres par le bout, aufquelles a 
pcine troune-t'on vn ou deux yeux de bons; vous la recueillirez proche du jet de l'année precedente, couperez le bout d'enhaut auquel vous ne pourrez prendre d'Efcuffuns, \& couperez auffit toutes les fueilles jufques à la maitié de lá queuë: ce que je vous oblige à couper le bour de la Greffe, \& les Fueilles jufques à la moitié de la queuë : \& que vous les laiffiez, en fe fanant, cela delifeicheroit tellement coute la Greffe, que l'on ne pourroit leuer les E[cuffons d'auec le bois, \& auffi que tout le fueillage vous cft inutil.

$S i$ vous ne greffez que le lendemain ou plufieurs jours aprés que vous les aurez cueillies, vous mettrez tremper le bout d'enbas dans quelque vaiffeau deux doigts de hauteur d'eau fuffifent) jufques à ce que vous les vouliez greffer, \&r fi vous voulez en grefferle mefme jour, il n'eft befoin que de les tenir fraifchement dans quelques Fueilles de Choux, ou linges moüillez.

Les Greffes pour la Fente fe cueilles dés le decours de la Lune de Ianuier, s'ap. pliquent au croiffant de celle de Fevrier: \& centinuant de Lune en Lune, jufques à ce que vous voyez que la Seue eftant trop forte dans le. Sauuageau en détache l'Efcorce d'allec le Bois. 
38 LE IAR D I N IER

Pour bien choifir la Greffe pour la Fente, mon opinion eft qu'il faut qu'il y ait du Bois des deux Seues de l'année precedente, dont le plus vieil fervira pour metre dans la Fente, \& le der. nier pouffera les Bourgeons; je ne defapproutue pas que l'on ne greffe auffi le Bois où il n'y aura qu'vre Seue, mais l'Arbren'en portera pas fi teft du Fruit.

Vous cueillerez vos (reffes au bout: des plus belles Branches, comme j'ay dit cy deuant, \& laifferez trois doigts de la premiere Seue, afin de tailler ailement voltre Greffe.

Pour les conferuer jufques à ce que vous greffiez, il fuffit de les enterrer à moitie tout en paquet, diftinguant pour tant les efpeces, de crainte que mettant deux Greffes de diuerfes fortes fur vn mefme Arbre, vous ne fufficz obligé d'en couper vn : d'autant que deux Fruits ne s'accordent jamais bien fur vn mefme pied, à caule que l'yn empelche l'autre de venir en la perfection, luy dérobant beaucoup de fa Seue. 


\section{De la maniere de Grffer. SECTION VI.}

TE n'ay remarqué que quatre manieres 1 de greffer qui foient necellaires, \& dont on puilfe efperer $7 n$ fuccez affeuré, les autres fortes eftant plus curieufes qu'vtiles; puifque par ces quatre on peut greffer toutes fortes d'Arbres \& Arbultes.

L'efcuffon tient le premier lieu d'autant qu'il s'applique fur toutes fortes dArbres \& Arbuftes, qu'il eft le plus facile à faire, \& rapporte plutoft du Fruit.

La Fente fuit apré; \& fe fait fur gros Arbres, \& fur petiss, jufques à vn poulce de diamettre.

La Couronne ne fe place guere que fur des Arbres bien forts.

Et l'A pproche ne fe pratique ordinairement que fur les Orengers, Citroniers \& autres plantes qui font dans des quaif. fes, lefquelles fe peuuent approcher \& joindre.

Four commencer donc par l'Elculfor, voftre Sauuageau eftant dépoiiillé de toutes petites branches jufque à la hauteus 
40 LE IAR D I IER

de demy pied, ou vn peu plus: dés le temps que lontailic les Arbies, ou bien à l'heure que voulez greffer, vous choifirez la plus belle place fur l'efcorce de roftre Arbre, \&r sil fe peut que ce foit du cofté des grands vents : parce qu'il en vient par fois de fi impetueux, qui décolent, les Efculfons à caufe de leur tendreur \& qu'ils font chargez de Feüilles \& de Bois; ce qui narriue pasfiordinairement quand ils font placez du colté des grands vents, que quand ils font de l'autre colté, quoy que vous y mettiez des pailfeaux pour les foultenir.

Vous taillerez voltre Efculion affez long, comme d'vn poulce ou enuiron, \& alfez large, affin qu'il prenne tant plus de nourriture : le leuerez proprement, \& regarderez par dedans fi le germe de l'œil y tient: Cars'il eftoit demeuré au bois, d'où vous l'auez leú, il ne vaudroit rien : vous le metrez à voftre bouche, en le tenant par le bout de la queuë de la Fueille que je vousay faic laiffer exprés en cueillant les Greffes, puis vous incifercz voltre Sautagean, \& leucrez douce ment l'Efcorce auec le manche de l'Entoir, rans frotter contre le bois, de crainte d'égratigner 
ia Scuc qui eft deffus, vous placercz voltre Efculton entre lc bois \& l'Efcorce, l'enfonçant jufques à ce que le haut de l'Efculfon fe joigne a 1 inciition d'enhaut de voftre Arbre, \& qu'il porte tout à plat contre le bois : cela fait thus le lierez ance du chanvre, eommençant à le ferrer bicn ferme par le haut prés de l'cil, puis en tournant par bas, laiffercz fort peu de jour à l'cil, nì finiflant voftre licure vous ferez le noud.

Frenez garde quand vous grefferez que ce ne foit ny pendant la grande ardeur du Solcil, ny durant le temps de piuye : car l'Efcuffon ne pcut fouffirir d'eftre moiillé, \& fera mefmement en grand danger de ne pas reprendre, s'il peut les quatre ou cing premiers jours enfuiuans que vous l'aurez greffé.

Il $y$ en a quien leuant l'Efcuffon, leucnt auffi dubois, cela fe faifant tout d'vn feul coup de coufteau, je ne defapproue pascette maniere de greffer, je m'en fuis bien trouvé ; car mes Greffes ont fort bien repris, \& de plus, on n'eft pas en danger d'éborgner vn Efcuf. fon, c'eft à dire de lailfer l'œil de l'Éculfon au bois de la Greffe; ceux qui ont quantité d'Arbres à greffer, fe feruiront 
42 LE I A D I N IER

de cette maniere, dautant qu'elle eft expediciue.

Trois femaines ou enuiron apres que vousaurez greff , vous couperez le nœud de voftre chanvre, afin que la Seue ait plus de paflage.

L'Hyuer eftant efcoulé, \& l'cil dormant conimençanta pouffer, vous couperez voftre Saluageau trois ou quatre doiges au deffus de l'Efculfon, \& couperez auffi la filalfe par derriere l'Efculfon iufques à l'Efcorce, cela fe fait d'vn feul coup de coufteau de bas en haut.

Vous n'ofterés point pourtant la filalle d'aucour de roftre Efcuffon, elle tombera allés d'elle mefme, \& puis il y a danger qu'en l'oftant l'on abbatte le Rourgeon qui eft alors extremenent teindre.

Quand voftre Efcufion aura poulfé cou te fa premicre Seue, vous le rongnerez afin qu'il jettedes Branches par lés yeux d'embas: autrement il monteroit fans. fourcher, \& parainfi voftre Naim n'auroit pas de grace.

Le vray temps pour l'arrefter eft vn Decours, auant que la Seue d'Aoult poulle : fi vous voulés en mefme temps vous couperez le bois du faunageau quaurćs laillè au delfus de l'Efculfon, 


$$
\text { FRA N çOI } 5 \text {. }
$$

Se coutriez la playe avec de la Terre franche meflée de Foin bien delié, faifant vne petice Poupéc: vous la pourrez coutrir plus proprement, avec vine (ire melée, dont je vous donneray la compolition cy aprés.

$\mathrm{Si}$ vous voulés attendre l. iffue de l' $\mathrm{Hy}-$ ner enfuiuant pour couper l'Argot de voftre Arbre, vous ne lerez pas obligé de l'enuelopper, car la Seure montant bien peu de temps aprés, la recouurira.

I’ay remarqué qu'vn Efculfon appliqué fur vn Saunageau, ou Franc, qui eit de la groffeur d'vin poulce, \& au deffus, ne pouffe pas fi bien que fur vn plus jeune, \& eft plus facile à décoler.

Il y en a qui efcuifonnent dés la premiere Seue, mais il n'auancent pas beaucoup; car l'Eiculfon ne poulfane qu' à la Seue d'Aourt, le jet n'en eft pas fi beau que celuy de l'œil dormant; d'autant que bien foument le Bois du nonueau jet ne meurit pas, \& l'Hyuervenant le fait mourir : c'eft pourquoy vous ne grefferés à la premiere Scue, fi ce n'elt vae grande neceffité.

Pour la Fente ou Poupée, tous Arbres depuis la groffeur du Poulce, jufques aux plus grands y peuuent eftre greffez; 
4.4 LE I A R D I NIER

le temps le plus propre, eft depuis le commencement de la Nouuclle Lune de Fevricr, jufques à ce que la Seue (eftant trop forte dans les Arbres) fepare le Bois d'aucc l'Efcorce, alors vous cefferés de greifer.

Quand vous grefferésen fente, fi c'eft pour faire vis Naim, il faut feicr vofte Saunageau a quatre ponlces ou enuiron prés de Terre; puis auec la Serpete ofter l'efpoiffcur d'vin Tefton du Bois, oì la Scie aura paffé, à caufe que le traica de Scie ne coupant pas nettement, la Seue ne pourruit recouturir ce Bois gratté, ny la Greffe $f e$ joindre all Tronc, fi fon Efcorce n'eftoit rafraichie auec la Scrpete : Cela fait, vous fendrés voftre Arbre par la partie où l'Efcorce paroiftra la plus vnie, \& moins nouieufe, \& obferverés de ne pas mettre voltre Serpette iuftement par le milieu de l'Arbre où eft le cœur du Eois, mais fort peu à cofté; puis vous taillerés voltre Greffe, en aiguifant tout le vieil bois jufques all noulueau en forme de Coin, également de chaque cofté, laiffant les dcux Efcorces attachées au Bois, car fi elles ne tenoient au Bois, la Greffe ne vaudroit rien; vous rognerés voftre Greffe a trois ou quatre poulces plus ou moins 


\section{FRA $N$ ÇOIs.}

felon fa force; d'autane que fur vn petit Saunageau, l'on n'en laifle pas de fi longuesque fur vn grand Arbre ? Cela fait vous olsurirés voitre Sauuageau auec le Coin, qui fera fais de quelque Bois dur comme Buys, Ebéne, ou autre, frapant doucement deflis, puis vous poferés voltre Greffe au bord du Sauuageau, en l'enfonçant jufques au nou. ueau Bois; \& faites en forte, que les endroits par où palfe la Seue, qui font entre le Bois, \& l'Efcorce de l'vn \& de l'autre, fe joignent.

Ayant pofé voltre Greffe, vous en incttres vne feconde de l'autre cofté de voftre Fente, obferuant de mettre toûjours deux Greffes à chaque Fente, pourueu que vous les y puiffiés placer fans fe toucher; car elles recouurent mieux leur Saunageau, \& plus promptement, que s'il n’y en auoit qu'vne, à caufe que la Seue monte également des deux coftés, sz ne laiflés mourir le derriere de l'Elcorce, comme j'ay dit cy-decant: En aprés, vous couurirés ce qui refte de Ia Finte cutre les deux Creffes auec vn peu d'Efcorce tendre, l'ajuftant curiculement, afin que l'Eau ne puiffe entrer dedans, puis vous ferés voftre Poupée ausc Terre franche, \& Foin bien 
46 LE IAR I I I ER

delié : a cuns pardulius la Poupée mettent de la moulfe, \& la font tenir auec deux Efcorces de Saule croifées, \& les lient d'vn Ozier au pied du Sauuageau, pour conferuer d'autant plus la fraif. cheur, \& empefcher I Eau d'y entrer.

Quand vous grefferés de grands Arbres, vous prendrés les Branches les plus vnies pour y placer vos Greffes: fi elles font grolfes, vous y en pourrés placer quatre, on fendant voftre Arbre en figure de Croix, fans pourtant toucher au cœur de 1 Arbre: les autres Branches que vous ne grefferés point feront fciées a demy poulce prés du Tronc, puis ayant ofté le Buts que la Scie aura gratié, vous les enmailloterés de I erre franche, \& ce aufil long-temps que $l^{3} E f$. corce fera à recouurir cette playe pour empefcher le hafle en Elté, \&a la gelée en Hyuer, qui entreroient par le cour de l'A bre à fon grand prejudice : il fera bon de lier quelques Efchalats aux rranches greffées pour entretenir les jeunes jocts \& les parer des grand vents, jufques aprés la leconde Aunée qu'ils feront affermis: \& s il Ce rencontre quelque branche qui poufe dagreablement, volis la couperés, comme aufi le tropá Branchus guis'eiturifient l’vue 
l'autre, donnane de l'Air au dedans de l'Arbre.

Et quand vous grefferés de petits Sau. uageaux qui n'auront pas la force de ferrer leurs Greffes, vous aiderés au Sau ageau à le ferrer, en le liant prés de la Greffe auec quelque petit brin d'Ozier.

Quoy que cy-deuant je vous aye obligé qu'à vos Greffes il n'y ait du Bois de deux Seues, neantmoins vous ne jetterés celuy où il n'y en aura qu'vne, ny auffi les rogneures de ceux ou aurés pris des Greffes de deux Seues, car ils Cont tres-bons, mais ils portent du fruit plus tard que les a tres, \& ne chargent pas tant, c'eft pourquoy fans neceffité vous ne vous feruirez que de ceux de deux Seues.

Le greffer en Couronne, ou entre le Bois \& l'Efcorce, ne fe fait point que fur de vieils Arbres, dont l'Efcorce endurcie pent Couffir le Coin fans eftre fenduë; \& lefquils ne feroient pas propres à faires vine fente (à caulé de l'efpoilfeur de l'Eicorce) fi ce n'eftoit à grande peine, \& cncor y auroit.il de lincerticude à la reprife.

Pour greffer en Couronne; ayan: fcié voltre Arbre à l'endroit où rous le yuil- 
49 LE IARDINIER

lés groffer, \& retailié le traict do la Scie iufque au vif, particulierement à l'cndroit de 1 Elcorce, vous taillerés vos Groffes par un feul cofté en aiguifant, puis frapperez vn petit coin de fer entre le Bois $\mathbb{Y}$ l'Efcorce, \&z ayant retiré voftre coin, vous y placerez la Greffe, en l'enfonçant jufques au haut de l'efguifure.

Vous en pourrez mettre autour du Tronc autant que vous youdrez, pouryeu que par la trop grande quantité l'Efcorce ne fe fende.

Quant au Greffe en Approche, il eft tres-facile à faire, car il n'y a qu' à pren¿re deux jeunes Branches, vne de Pranc, \& vne de Saunageau, fans les deftacher de leui pied, puis leur ofter à chacune enuiron quatre doigts le long d'Efcorce, \& de Bois, julques approchant du cœur, les joindre en femble le plus proprement qu'il fe pourra, les lier auec du Chan. ure depuis vn bout de la Taille jufques à l'autre: \& leur laiffer ainfi palfer les deux Seues : au bout d'vn mois, ou fix feraaines, fi vous voyés que le bois groffifle, \& que le Chanure l'incommode; St vous le couperés fur le Sauuageau, en tirant vn feul traict de coufteau, comme j'ay dit a l'Efculfon.

A u commencement de l'Hyuer faudra 
couperou feurer le framc de fa Mere, \& rogner le brtut du Saunageau à deux poulces prés de fon Greffe, par ainfices deux Brins ne faifans qu'vn Corps, le Franc prendra la nourriture du Sauuageau; Vous coturirez les playes de l'vn \& de l'autre auec la Cire que je vous enfeigneray cy-aprés.

' ous ne jetterez pas au Feu les Branches que vous aure? oftées des Coignaffiers qu'aurez greffezen Eente; car vous en pourrez faire la Bouture, qui dés la premicre année prendra racine, \& fera mife en Pepiniere pour eftre greffée en fon temps: ce que vous émondrés de vos Coignaffters durant l'Hyuer, fera auffi planté en Bouture.

Les Branches de Pommier de Paradis que 1 on appelle Eichet, reprennent auflis de Bouture.

Pour planter toute forte de Bouture, vous ferez vn petit Rayon, comme j'ay dit en la Pepiniere, (qui eft de la hauteur \& largeur du fer d'vne Befche) puis l'ayant bien efpluchée de petites Branches; \& taillée par le gros bout en forme de pied de Biche, ceft à dire à deux coups de Coufteau en appointant, vous 12 coucherez au fonds de voftre Rayon, la plaçant fort prés à prés, d'autant qu'il 
SO... LE I AR D I N I ER

en meurt beaucoup, \& ferez fortir le petit bout d'enhaut, puis remplirez le Rayon, \& le marcherez plufieurs fois en preffant la Bouture \& la pliant fort: autrement elle prendroit de l'éuent: \& quand vous la labourerez ce ne fera qu'auec la Binetre, ii fuffira que l'on empefche l'Herbe de l'eftoufer.

Vous rognerés voftre Bouture toute d'vne hauteur à trois doigts prés de Terre, \& ce quand vous verrez que la Seue commencera à s'émouuoir, faifant verdir les Bourgeons de voltre Bouture, laquelle ne poulfe jamais, fi toft que le Plan enraciné.

Des Arbres, of Arbufles en particulie", de leur gounernement cis remedes à levirs maladies.

\section{SEC TION VII.}

T'Ay creu qu'il eftoit neceffaire de faire I vn Chapitre feparé, qui comprift en détail tout ce que nous auons dit en gros aux Sections precedentes : \& ce pour éviter toute forte de confufion: afin que $\therefore$ il y a quelque chole qui yous falfe peine 


\section{FR A N ço I s.}

à comprendre, quoy que je me fois rifré de $m$ 'expliquer dans les termes les plus fimples, \& les plus communs de noltre Langue, pour eftre flus facilement entendu de toutes fortes de perfonnes, \& que chacun y puille profiter, que je le vous redife plus clairement, en particularifant fur chaque genre defruits, dont l'ont peuple le plus ordinairement les Iardins de noftre France.

En premier lieu, je mettray les Poiriers, comme portans le Fruit; dont il y a le plus d'eipeces que de tous les autres enfemble, \& defquels le Iardin eft principalement orné par les Efpaliers, Contr'Efpaliers, \& Buiffons, efquels l'on cueille des Fruits en leur maturité au moins pendant fix mois de l'année ; \& que ćeft le Fruit dont il y en a quantice qui fe garde jufques aux nouueaux, fans déchoir de la bonté de fon Gouft, ny fans fe flétrir; ce qui ne fe rencontre pas en tous les autres Fruits.

Tous Poiriers fe peuuent greffer des quatres façons que jay dites, ils viennent merueilleufement bien fur la Coignaffe \& en Elculfon : rapportent bcaucoup plutoft, le fruit citant plus bsau, 
S2 LEIARDINIER

plus peint, \& plus gros que greffez fur le Franc, refcrué le Portail qui manque foutent a reprendre fur la Coignafle, \& reut efre grefié fur le Franc ? Ie Graccioly, \& la Vallée y font tres-propres, \&z s'ils ont efté auparauant greffez fur la Coignalle, c'eft cncore mieux; car le fruit en viendra plus beau, is plus gros.

Si quelque Greffe en Efcufion, ou fente fur la Coignalfe, manque à pourfer faute d’eftre reprife, \& que vous jugiez qu'elle foit morte, lailfez repoulfer le Sauuageau, il jettera quantité de Bois que vous élaguerez de toutes perites Branches, \& l'Hyuer eftant à fa firı, vous ies butterez en forme de groffe Taulpiniere, lailfant fortir le bout des Branches fans les rogner; dés l'année mefme elles prendront Racines, pourueu que vous ayez foin de les arroufer dans les grandes Chaleurs, \& que vous ne laifLiez abattre la Terre par les Pluyes, la ieleuans à fa premiere hauteur ; \& fi dis l'année mefme vous troulez que quelques - vnes de ces Branches foient alfez fortes, vous les efculfonnerez, finon vous attendrez l'année d'apres pour les efcullonner toutes enfemble: \&z ce feront autant d'Arbres tous venus, que 


\section{F R A N ÇOIs.}

planterez en voftre Baftardiere l'année d'apres curil auront pouffé leur premier jet les feprant proprement de leur Me. re, \&e lis taillant au bout de de la grolfe Racine en pied de Biche.

Vous obferuerez de les greffer à demy pied de haut pour laiffer de la Tige à vofrre Arbre, qui prendra Cheuelure par tout où il fera enterré.

Si vous aucz des Meres Coignaffes, \& que vous en vouliez tirer des jeunes, vous plongerez les Branches dans Terre, 8. vne année fuffira pour les enraciner: I1 vous en voulez faire des Arbres tout d vin coup, vous le pourrez par la mefme maniere que je viens de vous monftrer. Le temps conuenable à plonger \& butter ces Branches, eft à la fin de l'Hyuer.

Les Pommierr tiendront le fecond lieu, \& fe peutuent auffi greffer de quatre façons, ils viennent fort bien fur le Fichet ou Pommier de Paradis, \& particulierement le $C_{a}$ luil y profite à merucille, \& eft plus rouge de dans, que celuy qui eft greffé fur le rranc.

Il y a guelques Curieux qui greffent le Caluil fur le Meurierblanc, \& tiennent que le Fruit furpalfe en rougcur tousles autres qui font greffez, ou fur Franc, ou

$$
\text { C iij }
$$


S4 LE IARDINIER

fur Fichet.

Les Pruniers ie greffent ordinairement en Efculfon, \& en Fente : in vous auez du Plan prouenant des Noyaux, ou des jetsque les Racines des Pruniers de Damas pourfent hors de Terre, vous en ferez de bons Arbres; car ils chargeront beaucoup, à caufe qu'il n'y a aucun Prunier tel qu'il foit, qui charge plus que celuy de Damas.

La Merizette ( qui eft vne elpece de Prunier Sauuage, dont le bout des Branches eft rouge) n'elt pas bien propre à eftre greffée : car elle refufe beaucoup de fruits, eftant fort incertaine à la reprife.

Les vieils Prunicrs qui font des Bourfes ou gros pacaners de menuës Branchos, ferone ra louis en les étctiant à la findel'Hyuer, \& ils poulferont du nouneau bois qui portera dans l'année fuiuante : vous ferez des Poupées de Terre \& Foin aux Branches coupées, \& rafraifchirez le traiot de la Scie, ainfi que j'ay dit par tout cy-deuant.

Les Abricots fe greffent en Fente \& en Efcuffon, fur le Plan prouenant de leurs Noyaux, \& fur le Prunier le Damas blanc, \& ceux de Moyeu d'ouf, font venir les Abricots plus beaux \& plus gros 


\section{F $\mathrm{R} A \mathrm{~N}$ ç $\mathrm{O}$ I $\mathrm{s}$.}

que fur les autres fortes de Pruniers.

Les Pefches, Prelles \& Pauies; fe greffent ordinairement en Efcuffon dormant, fur le Pefcher, Prunier, ou A mandier; mais le Prunier eft à preferer anx deux autres, car elles durent dauantage, \& refittent mieux aux Gelées \& mauuais Vents, qui roüillent les Füieit. les \& jeunes jets, les Pruniers blancs, ou Poictrons, n'y font pas bien propres, mais bien le Damas noir, Cypre, \& Saint Iulien ; ceux qui font greffez fur le Pefcher durent peu de temps : ceux fur l'Amandier durent beaucoup plus que les autres, \& font le fruit meilleur: mais il y a tant de difficulté à gouuerner l'Amandier en ce pays-cy, qu'il vaut mieux s'arrefter aus Pruniers : car l'Amand:er ne : eut point eftre tranfplanté, \& eft en d'anger de mourir, fil l'on attend plus d'vn $A_{n}$ aprés qu'il aura poulie fon premier jct, ou deux aninécs tout au plus, \& encore lc faudra il pla cer à demeurer, pour là clttre efcuffonne \& n'en plus partir: L'Amandier eft auffi plus fujet à la Gelée que les autres, à caufe qu'il fleurit plutoft : tout l'auantage que lon en peut efperer, eft qu'il ne produit point de Rejettons au picd. 
56 LF IA $R D I N I E R$

Les Cerifes, Bigarrentu, \& autres ruits femblables, fe gr.ffent fur le Merizier auec plus dauantage que fur autre Plan : le vray temps pour les efcuf fonner eft quand le fruit commence à rougir, \& prendre couleur : l'o a efcuf fonne auffi fur les Ceriziers qui tiennent du Sauuzge, \& dont le Fruit eft amer.

Ils fe greffent fort bien en Fente, \& pouffent de tres grande force. Mais l'Ee. culfon vaut beaucoup micux.

Les Figues de toutes forces, fe prennent de Marcottes pour plus grande facilité \& prompt raport; ce qui fe faiten paffant une jeune Branche de belle venuë, aे trauers vn boiffeau, ou mannequin, l'empliffant de bonne Terre meflée $d u$ petit fumi $r$ de vicille couche, afin qu'elle y prenne racine.

Vous prendrez garde de bien accofter, \& attacher le mannequin, de crainte que les vents, ou fa charge ne le renuerfent.

L'on prend auffi du Plan tout enraciné, des jets qui fortent de Terre au pied du Figuier; ou bien de la Bouture, que vous coucherez à la maniere que i ay dite des Coignaffiers, fans toutefois rien couper du haut de la branche que vous plongerez; carce bois qui a grande 
moëlle, craint fort l'éuent \& l'Eau : vous le plantercz à demcurer le plus jeune que vous pouricz, pour la feureté de la reprife.

A l'iffuë de l'Hyuer, vous ferez ofter de deffus les Figuiers toutes les Figues qui n'auront pas meury, fans les lailfer tomber d'elle mefme : car auant qu'el les fuffent tombées, elle auroient attiré beaucoup de la Seue de l'Arbre, au grand detrimenr des noutulles, qui faute de ce foin, bien fouuent ne peuuent s'acheuer de meurir.

D'autaut que les Figuiers craignent extrémement la Gelée, vous ferez obligé de les planter en quelque bon abry, oules metrre dans des quailfes, que l'on Serre l'hyuer auec les Orengers.

Des Orengers, \& Citronniers, je nen diray qué le principal \& plus commun gounernement, qui eft de femer des $\mathrm{Pe}$ pins dans des quaiffes, \& quand ils auront deux ans les replanter dans d'autres quaiffes chacun à part, lefquelles vous emplirez de bonne Terre, y meflant du petit fumier, \& de la Terre, glaize en poudre meurie par vn hyuer: quand ils feront affez forts, vous les efcuffonnerez ou grefferez en approshe dés la paemiere Seue, vous ferez foi- 
$58 \quad$ LE I A R DINIER

gneux fur tout de les preferuer du froid, les mettant de bonne heure dans la ferre, \& pour les garantir entierement de la gelée, ferez faire du feu de charbon dans voftre ferre, durant les plus grandes rigueurs de l'Hyuer, fi vous reconnoiffez qu'il y gele.

Au commencement du Prin-temps, quand vous croirez que les gelées feront entierement palfées, vous leur ferez prendre l'air petità petit, ouurant premierement la porte de la ferre à la plus grande chaleur du jour, \& la refermant la nuit : enfuite vous ouurirez les feneftres, que vous refermerez auffil $l_{2}$ nuit, \& les froids eftans entierement paffez, vousles fortirez de la ferre pour les mettre à l'air pendant tout l'Êté.

A mefure qu'ils groffiront, vous les changerez en des quailfes plus granảes, les leuant en mote, \& razant le petit bout des racines auec quelque confteau, puis les placerez dans les quaiffes, auec de la Terre de la mefme façon que j'ay dit: Aucuns en les changeant de quaifie en oftent toute la Terre la tenant pour vfée : mais cela détourne beaucoup de l'accroiffement de l'Arbre, car il eft vne année ou deux à fe faire.

Quant aux Fleurs, vous en pourrez 


\section{FrA c çOIs.}

cueillir tous les jours, de crainte quelles ne noüent à Fruit, ou que par trop épanouiies elles ne fe perdent : vous en laifferez feulement quelques - vnes des plus belles, \& des mieux placées pour auoir du Fruit; autant que vous jugerez l'Arbre en pounoir nourir.

Les araignées ayment fort à y baftir leurs toiles, à caufe que les mouches s'adonnent furleurs Fleurs \& Fevilles, attirées par leur bonne odeur, \& fuc: c eft pourquey l'on aura vne broffe femblable à celle dont on époudre les Tableaux, \& l'on lcs brollera legerement.

Les Arbutes le plus communs font, les Grenadiers, Ialfemins, Roziert-mufcats, \& autres, Cheurefueils, Myrthes, Lauriers ordinaires, Lauricrs-Ceriziers, Lauricrs-Rozes, Althea-frutex, Lilas, Rozes de Gneldres, Phylira ou Alaterne, \& beaucoup d'autres encor qu'il $\mathrm{fe}$ roit fuperflus de mettre icy, nous ferons feulement vn petit difcours fur les principaux.

Les Grenadiers, tant à Fleurs doubles quà̀ Fruit, fe marcottent en plongeant des branches dans Terre, \& les Laifrant ainfi palfer l'année, ils fe troumesont fuffifamment enracinez auant l'Hyuer, pour eftre tranfplantez: leur Bouture fera 
60 LEI AR DINIER

gouuernée comme celle des Coignaffiers: ils s'efcuflonnent, \& fe greffent aufif en Fente, en la faifon ordinaire : l'on en met dans des quaiffes, pour palfer plus feurement l'Hyuer dans la Serre: on en plante auffi en pleine Terre, contre quelque mur ou il y aura grand abry, car ils y viennent fort bien : les Grenades que l'on appelle de Raguiguan, font les plus rouges, \& les plus agreables au gouft, quoy que petites.

Les Ialfemins communs blancs, \& jaunes, fe marcottentauffi, \& ne font difficiles à gouuerner, venans en toutes Terres fans grande culture: l'onentire des Sauuageaux pour efculfonner le Jafmin d'Elpagne, qui veut eftre mis dans des quaiffes (à caufe qu'il eft fort tendre au froid) pour eftre (erré l'Hyuer auec les Orengers. Vous le taillerez tous les ans (a la finde l'Hywer) prés de fa Gref$\mathrm{fe}$, ne lailiant qu'vn ail à chaque brin pour produire les Fleurs, il ne Iuy faudra pas lailfer trop de charge, \& l'on formera l'Arbre comme vne petite tefte d'Ozier, luy laiffant vn pied de hauteur de rige.

Les Roziers Mufquars s'efculfonnent fur l'Efglantier, \& font de facile gou uernement : car il n'y a autre fujettion 


\section{FRA N C OIs.}

que les décharger du bois mort, \& arrefter les jeunes jets qui pouffent de trop grande force, \& qui attireroient à eux toute la Seue, laiffan: mourir les autres branches: il fe couche aufil dans Terre pour en tirer des marcottes, dont l'on fait des Arbres à part.

Les Mirthes, Lauriers-Cerifiers, \& Lauriers-Rofes, fe plongent auffi pour en tirer de marcottes, il fuffit que ce foit vn peu auparauant la Seue d'Aouft, $\&$ faut navrer ou fendre le bois que vous mettrez en Terre à l'endroit d'vn nœud, jufques à la moitié de la groflfeur de la branche, \& enuiron trois ou quatre doigts de longueur felon la force de la branche : en fix femaines ils jetteront vn cheuclu fuffifant pour les fevrer, \& tranfplanter : ils poulfent auffi du pied des petits $\mathrm{R}_{\text {amcaux tout enracinez, que yous }}$ pourrés feparer de la Mere.

Les Lauriers-Cerifiers fe peuuent mettre en Palliffades, \& palfent bien l'Hyuer en pleine Terre.

Ies Lauriers communs, fe fement de Palaufe, ou graine dedans des quailfes, comme les Orengers: \& dés la premiere, ou feconde année, fe replantent : fil'on les place fous quelque égouft de roict ( $\&$ non de goutiere ) à l'abry du Soleil de 
62 LEIARINIER midy, ils viendront beaux à merueille: Aucuns les cachent de grand foarre du. rant les gelées, aufquels il font fort fujets.

Le Philirea ou Alaterne, fe feme auffi dans des quailfes auani l'hyucr, \& font mifes dans la ferre, ou les graines germent \& pouffent beaucoup mieux, que fi ellesn'eftoient feméts qua au Printemps.

Quand ils ont feulement demy pied de haut, l'on les peut replanter, \& fouffrent d'eftre tondus comme les Buys, fans qu'il foient en d'anger de mourir.

Pour les aurres Arbuftes, comme Althea-frutex, Arbor-judx, Litas, \& f cmblables, eftant des Plantes faciles à íleuer $\&$ gounerner, je pafferay par deffus, craignant de groffir par trop noftre Volume, dont la lecture vous feroit peuteftre ennuyeufe, parlons plutoft des maladies des Arbres; \& des Aninjaux qui les incommodent.

De toutes les Maladies qui viennent aux Arbres, le chancre eft le plus dangereux : car il gerfe \& fait mourir la partie de l'L fcorce où il s'engendre, 8 va toufiours en augmentant fi l'on $\mathrm{ny}$ remedie promptement, auffi toft que l'on s'en apperçoit; fi bien que negligeant de vifiter curieufement les Arbres, 
on en trouue fouluent de tous morts d'vn cofté; pour remedier à ce mal, il le faut cerner tout au tour dans l'Efcorce viue, jufques au bois, le chancre tombera de luy-mefme, ou bien vous le gratierez afin que l'Efcorce recouture plus facilement la place, il faudra l'enueloper peur duhafle, mettant de la bouze de Vache fur le mal.

La Mouffe qui vient aux Ârbres prouient ordinairement de quelque caufe de deffous, qui eft que les Racines trouuent du Tuf, ou autre mefchante Terre, cu'elles ne penuent percer pour aller chercher la fraifcheur, \& par ainfi l'Arbre fe trovue bruflé \&e dépouillé de feuilles durant les grandes ehaleurs; il $n^{\prime} y$ a autre remede linon, que fi c ceft in pctit Arbre, il le faut leuer auec le plus de Terre que rous pourrez, \& luy faire vn beau trou de quarre pieds en quarré, mettre du fumier au fonds \& le remplir de Terre, \& petit fumier meflez enfemble ; a prés quoy vous replacerez l'Arbre obferwant ce que jay dit cy-deuant, \& il ne paroitt ra pas qu' il ait efté leué : car il reprendra Terre facilement, pourueu que vous ne laiffiez deffeicher \& éuenter fes $\mathrm{R}$ scines.

Si celt vn vieil Arbre, il faudra dé- 
64 LE IARDINIER chaulier auparauant l'Hyver, \& déterrer fes plus grolles Racines jufques à la moitié de leur groffeur, faifant vn grand cerne autour du pied de l'Arbre, \& le lailfer paffer ainfi tout l'Hyuer (ainfi que la Terre (e meuriffe) julques au renouueau que remplirés le trou, meflant quelque fumier bien confommé auec la Terre, particulierement le long des racines.

Vous gratterez la moulfe de vos grands Arbres, auec vne plane, en dofiant legerement leur Efcorce, \& celle des petits Arbres, auec vn coufteau rabbarti: su de bois: le temps le plus propre eft à l'iffuë de quelque pluye, ou à la rozée du matin: car durant la fecherefle, elle eft fi fort attachèe, que vous ne la fçauriez racler fans endommager beaucoup l'efcorce de l'Arbre, fi vous la vouliez gratter enticrement. Il ne faut negliger ce grattement, car lailfant lamouffe elle zugmente toufiours, \& eft aux Arbres, re qu'eft la galle aux animaux.

La jauniffe on langueur que l'on apperçoit aux fueilles des Arbres, eft caufeé par quelque bleifures, que les Taulpes \&z Mulots petiuent atioir faites en leurs racines, ou par le fer de la befche, ou bien qu'elles font fuffoquées par trop 
d'eat qui y croupit.

Pour y remedicr, il faut déchauffer l'Arbre de tous coltés, \& le vifiter, fi vous trounez que les Taulpes, Mulots, ou la befche l'ayent efcorché, ou fait quelque dommage en fes racines, vous les couperés bien vniment en pied de Biche, au deffus \& proche de la pluye, puis mettrés au fonds du trou des fuïes de cheminée, pour étranger ces beftioles, \& le remplirés de bonne Terre meflée de potit fumier : \& ficefont les eaur qui y croupiffint, il ne faut que faire vie tranchés plus baffe pour les écouler.

Pour prendre les Taulpes, aucuns enfoiiiliTent dans la Terre vin pot à beure au chemin par lequel elles ont acconftumé de palfer, enfonçant le pot deux doigts au deffous de leur trace, $\&$ en paffant elles fe laiffent tomber dedans ce pot. Autres fe feruent d'vn canon de bois de la groffeur du bras prés le poignet, \& de la longucur de deux pieds, lequel a vine petite languette de fer blanc, à quatre doigets prés de chaque bout, qui eft attachée au canon auce vin fil de fer, vn peu penchante par lc bas vers lo milieu du canon, afin que la Tauipe c1ntrant dedans, \& pouifant la langucte 
$66^{\circ}$ LE IARDINIER n'en puiffe plus refortir, ny par vn bout, ny par l'autre: l'on le mettra iufte au chemin que l'on aura veu, nouneau tra. cé, les deux bouts refpondans le plus proprement que l'on pourra aux deux trous qui feront à la trace: d'autres pour eftranger ces animaux de quelquiendroit cù l'on les verroit bien obltinez, font vne petice enceinte de bois de Sureau, qu'ils fichent dans Terre à demy pied de profondeur : le plus feur eft de guet. ter le matin \& le foir, quand elles trauaillent à faire des buttereaux, ou taulpinieres, \& les enleuer adroitementáuec la befche: fi vousen prenezquelqu'vne en vie, vous la mettrez dans le pot à beurre, car l'on tient que par leur petit cry elles appellent les autres, quivenane parla trace déja faite, ne manquentà fe lailfer cheoir dans le pot.

On les affomme auec vn maillet où il y aura des pointes de cloud longues d'vn doigt ( lequel fera emmanché vn peu long en frapant furla taulpiniere, alors qu'elles trauaillent, \& foiillant promptement auec la befche, l'on ne manquera de les trouuer percées, ou eftourdies, ou mertes.

Les Mulots fe prennent en faifant auec du foarre vine petite hutte, comme la 


$$
\text { FRA } \mathrm{N} \text { çOIs. }
$$

conuerture d'vne ruche, mettant deffous quelque terrine, on chaudron plain d'eau jufques à quarre dinigts prés du bord \& jeter par delfus l'exu vn peu de paille d'Aucine pour la cacher, ils y viendront pour fe veaurer ou y chercher quelque grain \& ils le noyeront, il eft bon aufit de metre des clanes de Bled ou d'Auoine : qui pendent au milieu du chau. dront fans y toucher, car ils iront pour manger \& ie lailleront tomber dans l'eau.

Si vous voulez les empoifonner, l'Arfenic broyé mis dans quelque grailfe les fera mourir, mais il eft dangereux que vos chats lestrouuant morts ne les man. gent, ce euilesferoit au Mt mourir, fice n'cftoient aे l'heure melme, is languiroient quelque temps \& cutin : ${ }^{2} \in r_{s}$ éciaperoient pas.

Les vers fe mettent quelque-fois entre le bois \& l'efcorce de l'A rbre, fi vous poutiez juger où ils font pour les tirer fans faire grande incition, vous les ofterez proprement.

Il y a d'vne autre efpece de petits yers que l'on appelle coupe-bourgeons, qui $s^{2}$ engendrent au bout des jeunes jets, \& quifont mourir tout le haut : pour cenxlà ils font allez ailés à trouucr, cu cou- 
68 LE IARDINIER

pant la jeune branche jufques au vif, vous ne manquerez de les rencontrer.

Les puceons-vers qui mangent les jeunes $j$ ets à mefure qu'ils poriltent, font fort difficiles à eftrancher, li ce n'eft en la barboüillant de chaux viue recentement efteinte: vue brolle de Peintre de la grofieur d'vin bon poulce, fera propre à cét effet.

Les fourmis s'cftrangeront de l'Arbre où ils sattachent, fi vous y faitcs vne ceinture au Tronc de le largeur de quatre doigts auec la Laine fraichement tirée de deffous le ventre d'vn mouton: oil fi vous le graiffez de parcille largeur auec du tare, qui eft vne gomme noire de laguelle on frotte la galle des moutons : il y a vn autre moyen moins inle \& plusfacile, qui eft de faire dies petits coffrets de carte percez en plaficurs endroits auec vn Poinçon, \& que dedans il y ait de l'appas fait auec arî́nic broyé bien dclié, \&z miel meflez enfemble : ces coffrets feront pendus à l'Arbre, \& les fourmis allant manger cét apas, s'empoifonncront, \& mourront : vous prendrez garde de ne faire les trous fi grands, que les mouches miel y puiffententrer, car elles mourroient auffi.

Vne bouteille de verre dans laquelle il 


\section{Fr A ș ç O I s.}

y aura eu du miel, ou autre liqueur fucrée, fera attachée à l'Arbre, tous les fourmis entreront dedans vous la boutcherez, \& la porterez à la mailon, vì là auec de leau chaude vous la lauerez, \& tuërez tous les fourmis, puis vous y remeitrez quelque liqueur fucré, \&la reporterezà l'Arbre, ce failant vous tuë. rez jufques au dernier fourmy.

Les limats à coquille feront facilement pris derriere les fueilles les plus proches des Fruits, que vous verrez qu'ils auront manchez pendant la nuit : car il fe troulue par fois des Fruits qui font mangez jufques à la moitié en vne feule nuit, l'on croit que c'eft la Fouiine, \& les Rats verets, ou Lers: mais ce ne font que des limats, qui eftansen grand nombre mangent autant qu'vn de cesanimaux.

Vous ne détacherez de l'Arbre les Fruits que les limats ou autres beftioles auront entamé, car ils ne toucheront aux autres qu'apres que ceux-là feront entierement mangez.

Il $y$ a d'autres limats - noirs fans coquille, qui font faciles à oiter : car ils sattachent au delfus des fueilles o rongent.

lour ce qui eft des cloportes, perceoreilles, artinets, 8 autres petits in- 


\section{LE JARDINIER}

fectes qui gaftent les Arbres: On mettra des orgles de Bøuf, Mouton, \& Pore au bout de pieux, ou fur les ozicrs qui lient les Efpaliers, \& Contr Efpalicrs: dés le grand matin deux hommes iront à cette chaffe atlec vin chauderont, l'vin leuera doucement, mais promptement ces ongles, \& les frapant au dedans di chaudront, cès beftioles tomberont, \& l'autre les écrafera auec vn pilon de bois, de crainte qu'elles ne fe fautent.

Quant aux chenilles, elles lont tresfaciles à enleuer durant tout 1 Hyuer, oltant les pacquets que l'on verra aux branches des Arbres, \&z les jettant au teu : ce que vous auriez grande peine a faire, fi vous les laiffiez éclorre : \& en cas que vous en ayez oublié quelquesvns par mégarde, \& qu'ils éclofent, ne negligez pas de les ofter en leur jeuneffc: quaud par la fraícheur de la nuit, ou par quelque humidité, elles feront amallées tolites eft vn monceau : car au grand Soleil, \& au haut du jour, elles ie difperfent par tout à l'Arbre.

Vous quitterez route forte de befogne pour courir à celle-là, comme la plus neceffaire de tout le jurdin, quiayant efté aftligé de ce venin-là vne année, s’en reflent plus de trois autres aprés. 


\section{FRA N ç OIs.}

Il ne relte plus de ce 'rraicté, quª̀ vous donner la recepte que je vous ay promife pour compoler la Cire à couurir les Entes: Vous prendrezdemie liure de Cire neufue, autant de poix de Bourgogne, \& deux onces de Tharebentine commune: vous firez fondie le tout enfemble dans vn pot neuf, de Tcrie, qui foit vernilfé, cn les remuans fou. uent : vous lailferez refroidir cete com. pofition au moins douzz heures, uis vous la romprez par morceaux, la tenant dans l'eau tiede lefpace de demieheure, la maniant 2 érompantentierement, pour eftre plus facile à appliquer: Vous pourrez aufil tremper de 12 Toile dans cette compolicion, qute couperez commes cmplateree propres à la playe de vos Arbres \& cela vous épargnera peaucoup de cette compolition, d'autant qu'il n'y en entrera pas tant qu'en morceaux : Vous vous leruirés auffi de cetie Toile pour conurit la fente de vos Arbres qui roftent entre les deux Greffes, pour la preferucr de l'eau; $\&$ vous en enucloperés aufli la Poupée, auant que de mettre la Terre \& le Foin, cela vous affeurera que l'Eau ne pourra nuire à voltre Greffe.

Il y a quelques curicux, qui pourfaire 
72 LE IARDINIER

noiier \& arrefter les Fruits aux Arbres qui ne font que flcurir, percent de part en part le tronc de l'Arbre par le milieu auec vne Tartiere de la grolleur du doigt, \& frappent dedans vne cheuille de bois de chefne tout à trauers: ils tiennent que cela arrefte le Fruit : Efprouuez le fi vous voulés, la peine n'en eft pas grande, \&z vous ne hazarderepoint vofre Arbre.

\section{CATALOGVE DES NOMS}

Des Fruits dont nous auons la connoiffance au Climat de Paris.

Poines derauoiles le Frait of en Sa maturité dans la fin du mois de I ain of Inillet.

PEtit Blanquet.

1 Haficicau de plufieurs fortes. Nufcadille, ou fept en gueule. Sainc Ican mufqué.

\section{En Iwillet, \& Aouft.}

Ros Ariyret. Petic Amiret. Amiret loannet. 


\section{FRA NÇOIs.}

Bonnes deux fois l'An.

Camouzines.

Chere à Dame mufqué.

Chere à Dame vert.

Citron.

Coquin Rozat.

Cuille Madame.

De Maderes.

Defgrange jaune.

Deux Teftes.

Douces de deux fortes.

Du Vacher Rozatte.

Elpargne.

Fin Or a longue queuë.

Fin Or d'Orleans.

Fin Or, gros, rond \& rozat.

Friquet.

Glouttes de Gap.

Magdelaine.

Mulguat à longue queué.

Mufquat en perle.

Grofie mufquée blanche \& jausse.

Grofle Muzette.

Petice Muzette.

Perdreau.

De Peale.

Pernant Rozat.

Proutence.

Pucelle de Xainatonge.

Realles vertes. 
7.7 LEIARDINIEK

Rozatte de trois Couleurs.

Rozatte rouge frotée de vert.

Royales Rozartes.

Roy d'ERté.

\section{E: Aonf, \& Siptembie.}

A Mazones.

A Amours.

Amydon.

Armentieres.

Baulme.

Beau-Pere.

Belles \& bonnes.

Bergamotte d'Elté.

Gros Blanquer.

Bœurée d'Aouft de longues \& de rond:s。

Bæurée verte.

De la Beuneriere.

Bezy de Mounilliers.

in Clrzttien d’Efté vert.

Bon Micet de Coycux.

Bructe-bonne.

Cadet.

Caillon rozat mufqué.

Chitio de fille.

De Circ.

Cine.

Conll-foif, ou Munile-bouche.

Dhack: asoter. 


\section{FR A t c O I 5 .}

Dorées.

Eicholettes blanches.

Efpice.

Ecreft.

De Folle.

Fourmy-mufqué.

Galleufes.

Garbot rofat.

Galteau.

Giacciole di Roma.

Gillerte-Iongue.

Graccioli rouge \& rond.

Gralles.

Ialoufie.

Iagonnelle.

Ioviars.

De merueille rouge \& jaune.

Milan.

Mufcadelle de Piedmont.

Mufcar rond \& rozat.

Mufcates de Nançay.

Nounelet d'Efté.

Oiginon d'Elté.

Oignonnet mufqué.

D'Or.

Orenge de Xainctonge rouge, fort grofe fe.

Orence jaune peninachie, de rouge en Trilipes.

Orenge noites en bigurades.

$D \mathrm{ij}$ 
76 LEIARDINIER

Orenge plat \& vert.

De Palme Inle de Canarie.

Palfe-bon de Bourgogne.

Papin.

Piedmont blanches \& rouges.

Portugal d'Efté.

Pures.

Rozattes de Xainctonge de trois fortes.

Rozatte d'Ingrande.

Rozatte ronde, verte \& rouge mélée.

Rozatte roulfe de Xainctonge.

De Rozes ou Bouré haftif.

Sanguinolles.

Sauuages douces.

Soreau.

Sucre.

Sucrin blanc。

Treforiere.

Trompe-friand.

Turquie.

Vallée.

Vilaine d'Anjou.

Vilaine de la Reatte.

\section{En Sepieinbre, or Ociobre.}
N Ncy:
A. Angleterre.
Bec d'Oye.
Rourée longue \&f verte. 


\section{F R A N C O Is。}

Caillouiat de Champagne.

De Caluile mufqué.

Canelle.

Cappor..

Clairville longue.

Certeau d'Esté.

Crapault.

Doyenné:

Efpine.

Fontarabie.

Galoré.

Girofla.

De Girofie rond.

Grain.

Guamont Rozatte:

Haute-faueur.

Iargonnelle d'Automne.

Kerville Rozatte.

Gros Lichefrion.

Petit Lichefrion.

Lombardye.

De Mailleraye.

Aux Moufches ou Bouré tendre.

Be Monfieur.

Petit Moiille bouche.

Mufquée.

De la Moutieres de Dauphniné:

Oignon de Xainetonge.

Poidiers.

Rebers. 
78 LE IARDINIER

Roland:

Gros Roulfelet de Rheims.

Pctit Rouffelet.

Rozattes longues femées de rouges.

Deux fortes de Rozattesvertes.

De S. Michel.

De. S. Sanfon.

Sans nom de Champagne.

Saulfinottes.

Septembre Rozattes.

Suprémes.

De trois goufts.

Trouuées.

De Vendanges.

$Y$ fambert.

I velin.

\section{En Octobre, í Nouembre:}

\section{Madotte. \\ A D'Argent.}

Cornemufe.

Glaffe.

Groffe-queuë.

Laide bonne.

De Madame.

Marion d'Amiens.

Moffire Iean vert.

Meffire Iean gris.

De Milord. 
Petit Mouiille-bouché d'Automne.

De Pefches.

Pucelle de Flandre.

Double Pucelle.

Robine.

Roy de Saulçay.

Roy mufqué tout jaune.

Saffran Automnal.

De Seigneur.

De Soleil.

Tant-bonnes..

De Vigne.

Virgoulette.

\section{En Nouembre, \& Decembre.}

\section{A Leaume.}

1 Bergammote mulqué long.

Bergammotte rond.

Bezy-Dairy.

Carify.

Cartelle double.

Chat brûlé.

De la Charité.

Eitouppes.

Fuzée.

Girogille.

De Noltre-Dame.

Orenge d'Automne.

Pucelle d'Hyuer. 
So LE I A RDINI É Tú.

Roy Automnal.

Sans Pair.

Sucrin blanc.

Sucrin noir.

\section{En Decembre, of Ianisier.}

Nonymes.

D. Bergamottes de Gafcogne.

Eon-Chreftien Mufqué.

Bonnefoy.

Eadillac.

Carteau Madare.

De Liure.

Efcarlatte.

Figue.

Franc-Real.

Gros Mefrils.

Martin fec.

Meffire Iean d'Hyuer.

Milan.

Oygnonnet ì courte-quew:

D'Orient.

Plomb.

Roy Roux.

Saffran rozat.

S. Denis rozat.

Santé.

De faulciffon, femblable à vn ceruelas. Torture de deux fortes. 
Trompe-coquin.

FRAN दुOIS.

Voye aux Preftres.

En Ianvier, of Fevrie\%.

A. Lençon.

A. D'Ambre.

D'Amours.

Bezy de Priuilliet.

Bezy de Qualfoy.

Beuréz d'Hyuer de Xainctonge.

Baitrée des Yueteaux.

Rouvart.

Caillotet Mufqué.

Cailloiiat de Varennes.

Cailiou rozat d'Hyuer.

Carcalfonne.

Gros certeau.

Petit certeau croche.

Chalteatr Gontier.

De condor.

Petit Dagobert.

Dagobert de Mioltan.

Dame houdotte, Poire de graine.

Elchelettes rouges.

Fin Or d'Hyuer.

Florentine rozate.

Fremont.

Frazée d'Hyuer.

Garay d'Auxois: - 
82 LE IAR DINIER:

Gourmandine.

Hongrie tres grofle.

Legat d'Hyuer.

Limon doux.

Longue Verte de Berny.

Micet.

Mouille bouche d'Hyner.

Mufcat à queuë de chair.

Mufquat de Mezeray.

Muzette d'Hyuer.

Nanterre.

Oignon de S. Ieand'Angely.

Orenge d'Hyuer.

Perigord rozatte.

Plotot.

Portail.

De Prince.

De Prunay prés Sillery.

Rabu blanc.

Ratot gros \& petit.

Rozatte de Xainctonge.

Rozatte de la Mafuere.

Suilles à bandes rouges, vertes, \& jaznes.

Verdureau.

Tillaines d'Hyuer. 
En Frvicr, o autres Mois Suinans infques aux nounelles.

\section{REzy.}

DBon-Chreftien tardif.

Gros Chreftien.

Calo rozat.

Chefne-gallon de plufieurs fortes.

Double-fleur.

Gaftelier.

Gros Kairuille.

Liquet.

Longue-vie.

Longue-vertes.

Mufc.

Parmein.

Pucelle d'Hyuer.

Rille.

Saffran d'Hyuer.

Sans P.air.

De Thol.

Gros Trouué. Petit Trouué.

\section{Pommiers bafiffs.}

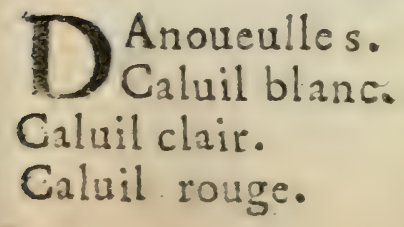


8:4 LE IAR D INIER.

Comoifes blanches.

Carmagnolles.

Chataignier tendre.

Cliquet.

Petit Courpendi rouge.

Gros Coufinot.

Coufinotes longues.

Confinotes rondes.

L'Enfer, ou Noires.

Efcarlatte.

Efpice.

Fleur de May.

Framboisée.

Giradottes.

Glacées.

Gros œil.

De Iacob.

Lugelles.

Magdelaine.

Mignonne.

De Neige.

Noftre-Dame.

Oblongues litî́es.

Orgeran.

Pallepommes de plafiensefpects.

Pommaffes

Rambourg blanc.

Rambourg rouge.

Reinette hafiuse.

Royales. 
Rozée.

Grolle rouge de Septembre.

Rouges tendres.

S. Iean de deux fortes,

Pommes à troches.

De Vignancourt.

Violettes de Mars.

\section{Fommes de Gavde.}

Ros Apis.

IfFetit Apis.

Apicles.

Apium.

Babichet.

Gros blanc.

Blanches Glacées.

petit Bon.

De Pretagne bianc.

De Bretagno rouge.

De Cardinal.

Camueras.

Chanaigner d'Hyuer.

Citron.

Coqueret de plufieurs fortes.

Courpendu dur.

Courpendu rouge.

Courpenda roux.

Dieu.

Duiictes. 
36 LE IAR DIN ER

Drap d'Or de Brctagne.

Eftrangeres.

Fenoiiillet blanc.

Fenouiillet rous.

De Fer.

Groffe Femme.

Haute bonté.

Hurluua.

Iayet.

De Iudée.

De Malingeres:

Mattranges.

Palfe-Pommes d'Hyuer.

Pigeonnet.

Poires-Pommes.

Raënlées.

Reinette d'Autuergne.

Reinette de Mafcons.

Reinette grife.

Reinette platte.

Robillard.

Rouzeau d'Fyuer.

De Rofes.

Pommes fans fleurir.

Santé.

De Scigneur.

Vermillon. 


$$
\text { F. A A ço Is. }
$$

\section{Prunious baftifs on andifs.}

Aricotées.

A Abricotines.

Ambre.

Gros appetit.

Beffonne.

Blanches de Toufraints.

Bloffes.

Bonnes à Noël.

Brignolles de Prouence. Brignolles citronnées.

Cerizettes blanches,

Cerizettes rouges. -

Citron rondes.

Citron pointuës.

Cœur de Pigeon.

De Cypre.

D'Amandes.

Gros Damas blanc.

Gros Damas double.

Damas gris tardif.

Damas noir haftif.

Damas noir mufqué.

Damas violet.

Dattes blanches.

Dattes rouges.

Grofle Datille.

Dariltes. 
38 LEIARDINIER:

Diaprée blanche \& noire.

D'Efcarcelle.

A fleur double.

Haute bonté.

Grolfe Imperiale.

Imperiale rond.

De Ionuille.

Iorafes.

Inleuert.

Maximilianes.

De Merville.

Mirabelles.

Mirabolans.

Miroir.

Moyeu d'œuf.

Moyeu de Bourgogne.

De Monfieur.

Montmiret.

Mufquée.

Paffe velous de Valcnçay.

Perdrigon blanc.

Perdrigon noir.

Perdrigon rouge.

Perdrigon tardif.

Perdrigon vert.

Trés-gros Perdrigon violet.

Poictron.

Raifnettes ou Prunes à grappes.

De la Reine Claude.

Rognon de Cog. 
De Rome.

Rondes tardiues.

Roy de Breffe.

S. Anthonin.

Sainte Catherine.

S. Cir.

S. Iulien blanc.

S. Iulien noir.

Grolfes Saluces de deux fortes.

Sans noyau.

Simiennes.

Trudennes noires.

Trudennes rouges.

Des Vaccations.

De Vendangę noires.

Verdaces.

\section{Pechors fo Pavies.}

Rofles Alberges.

Petites Alberges.

Alberges de Prouence.

Aubiçons.

Pelches Amandes.

Pauics d'Ambre.

Angeliques.

Auant-Pefches blanches.

Auant-Pefches jaunes.

Gros Brignons de Bearn.

Brignons mufquez. 
90 LEIARDINIER

Pefches Cerifes.

P.efches de corbeil.

Dure d'Hyuer.

A fleur double.

De Gaillon trés-belles.

Pauies jaunes.

Pauies de Magdelaine.

Pefches de Magdelaine.

Mircotons blancs.

Mircotons jaunes.

Mircotons de Iarnae.

Pefches-noix.

Parcouppes.

De Pau.

Pefches-prunes.

Pauies-raues.

Pefches-raues.

Perfiques.

Perfilles.

Roffanes.

Scandalis blanc.

Scandalis noir.

Toute jaune.

De Troye.

De vigne fromentée.

Violettes. 


$$
\text { FRA N Ç O I } S_{0}
$$

\section{Cerifes, Bigarreaux of femblables.}

Rigarreaus.

BCerifes blanches.

Cerifier à fleur double.

Cœurs.

Griottes.

Grindoux.

Guines blanches.-

Guines noires.

Merizier à fleur double.

De Montmorency à courte queuë.

Precoces.

A trochets.

De Toulfaints.

Figues.

Buanches.

Bourno-Saintes.

Fleur.

Gourrauau de Languedoc,

De Marfeilles.

Naines blanches.

Naines violettes.

Blanches.

Violettes. 


\section{LE IARDINIEI \\ Orengers.}

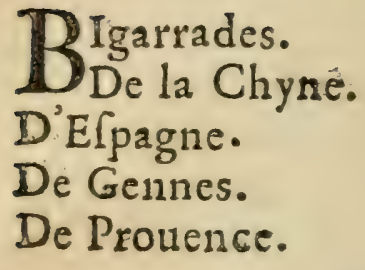

Citronso-

TImonchailli.

LLimoni Cedri.

Limoni Dorfi.

Limoni di Gauita。

Limon doux.

Pommes d'Adam.

Poncilles.

Spada fora à feúililes de Lauriex:

Autres Arbres curieux.

Rboufier.
Azarollier.

Carroubier.

Cornouiller.

Iuiubier.

Mirabolans d'Affrique.

Neffles fans noyau.

Piftachier.

Vinottier fans pepin. 


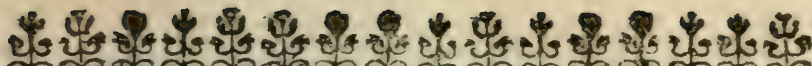

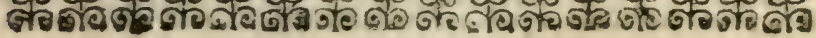

\section{E}

I A R D IN IER

F.R A N C O IS

j

SECOND TRAITTE:

Des Melons, Comcombres, Citroüilies, er autres efpeces femblables.

SECTION PREMIERE.

TVIs $V$. E les Melons font les plus precieux Fruits que vous pouuez 1 recueillir de vottre Iardin Potager: Il eft fort à propos de les mettre en tefte de ce Chapiere, \& rous donnervine inftruetion trés-ample de quelle façon ils fe doiueut gounerner, particulicrement au Climat de Paris, qui eft celuy duquel je me fuis propofé de vous eferire, laiffant les autres qui par leur difference vous pourroient apporter du doute, 


\section{LE IARIINIER}

fi je confondois la maniere qu'ils s'éleLent is Pais chauds, auec celle du no ftre qui eft temperé \& plutoit froid à l'égard de ces Fruits delicats.

Pour paruenir à uoftre intention, qui eft de les zuoir bons, vous ferez foigneux de rechercher des Graines de tous coftez (d'ltalie, de Lion, de Tours, d'Anjou, de Champagne, \& autres lieux où on fe pique de bonne Melonniere) \& de toutes les efpeces, comme fucrins, Morins, Melonnes, Grenots, biancs, outuragés, ou brodés, langés \& autres, merme de ferrer colles dont aurés mangé le Fruit qui vous aura femblé de bonne Seue : car tel les aime d'vin gouft, que l'autre rejettera \& tiendra pour defagreable; l'vn ainera à les manger vn peir verds, \& l'autre les voudra fort neurs; $c^{2}$ eft pourquioy vous vous en fournirès des elpeces où fe portera particu-. licrement voltre appetit, \& remarquerés ceux que voftre Terre nourrit le mieux, E meurit le plutoft; $c^{3}$ eft où vous vilerezlur tout, carbien foument dés le mois d'houft il vient des pluyes qui les perdent enticrement, les rendans lans odeur, fans faucur, fans coulcur, pleins d'eau, incapables d'eftre mangés, \&r meuriflans tous d'vn melmetemps, n'etrans propres 
qu'à donner aux Cheuaux qui les aiment extremement; bref ces pluyes dépoiiilo lent, \& font celfer voltre melonniere, où l'on aura pris tant de foin; \& le trauail de cing ou fix mois fe trounera perdu, fans en auoir eu le contentement qu vous en efperiez : C'eft pourquoy, il vous faut effayer d'en auoir de bonne heure pour preuenir ces mefcontentemens.

Aux païs où l'on en fait beaucoup auec peu de foin, les mettans en pleine Terre comme l'on feroit vn chou, fit toft qu' if eft venu des pluyes ils n'en mangent plus, \& croyent que ce leur feroit du poizon.

Pour commencer done voftre melon. niere, vous choilirés dans l'enceinte de voftre Iardin le lieu le plus à l'Abry du mauuais vent, que vous clorrés d'vne haye faire auec du foarre long, lié par paillartons, \& que volis affermirés \& arrefterés bien auec de bons picux fichez daus Terre, \& accoftez, de crainte qu'ellune foir renuerfée par les vents; il y ausa vie porte pour 1 entrée, qui fermera à clef, empefchant que toutes fortes de perfonnes n'y aillent toucher, \& particulicrement pour en interdire l'entrée aux Filles \& Fommes, encertain temps que le refpect m'ompefcle de de lare: 
9:6 IE IARDINIER

La figure qui eft au commencemene de ce Traité, vous fera facilement comprendre de guelle maniere vous deuez clorre voftre melonniere.

Dans ce parc qui fera de telle grandeur que defirerés auoir plus ou moins de Melons, vous ferés deg couches de fumier de cheual, qu'aurés amalfé durant l'Hyuer, à mefure que l'on le tire de l'Efcurie, \& que vous aurès fait metcre en monceau proche de voftre melonniere.

Dés le mois de Feurier, vous commencerés par dreffer vne couche pour les $\mathrm{Se}$ mences, prenant du fumier fortant tout chaud de deffous les cheuaux, \& de celuy qui fera en monceau les entremeflant enfemble, afin que le chaud rechauffe l'autre.

Vous ferés voltre couche toute de la longueur de la melonniere, de quatre pieds de larga feulcment, laiflant vn fentier tout autour de trois pieds de large pour y remertre du fumier chaud quand vous vous apperceurés qu'elle pourroit atioir parie toute fa chaleur, \& qu'elle fut morfonduë.

Cette couche eftant drefléc bicn également, \&z pilée auec les pieds pour la faire échauffer; vous mettrés par dellus cnuiron quatre poulces do liaut de bon 
terras ou petit fumier de vieille couche mené auec quelque peu de la meilleure Terre que vous ayés; vous la drelferez tout à l'entour en tenant vn ais fur le cofté au boid de la couche, \& foulant vn peu de terras auec la main contre l'ais, fon l'affermira $\&$ le drelfera-on vniment.

Voltre conche ou forme eftant achenée, \& de la hauteur de trois pieds ola cnviron, vous la laifferez échauffer \& paffer ta plus grande chaleur, qui durera deux nit trois jours, plus ou moins, fclon que le temps y fera propre: La force de fa chaleur eftant paifée, ce que vous reconnoinrez par la couche qui s af. felfera, \& auffi en fourant voftre doigt dans le tcrras, vous jugerez fi clle eft propre à femcr, car fi vous n'y poutuez durer le doigt, elle eft encore trop chaude : il faut qu'elle foit feiche, mais non pas toute froide, \& en cas qu'clle euft palfé toute fa chaleur \& fut morfonduè, vous la rechaufferez, en mettant dans les fentiers du fumier tiré chaud de deffous les cheuaux, comme je viens de dire.

La couche eltant en bon stat, \& voftre graine ayant trempé deux fois vingt quatre heures dans de bon vin ou sinaigre, ou bien du laict de vache, mettant chaque clpece a part, vous la ferne-

E 
98 LE IA R D I N IER

rez fur vin bout de voltre couche, referuant le refte pour les autres graines que je diray cy-aprés.

Vous ferez fur voftre terras de petits traits auec le doigt en trauers de voftre couche, diftans de fix poulces, \& les plus droits que vous pourrez; on bien vous prendrez vin bafton bien droit, \& le pofant fur voftre couche appuyerez delfus, il marquera fuffifamment le trait.

Sur chaque trait, vous ferez fix trous dans voitre terras, en enfonçant le bout de vos doigis joints enfemble en forme de cul de poule; \& dans chaque trou y mettrez trois ou quatre grains de melon, tout d'vne efpece.

Sur l'interuale de cét efpace de demy pied que je vous ay fait laiffer, vons y femerez de la laictuë pour manger en falades nounellcs, \& du cerfueil en quelques vnes: Vous femerez auffi du pourpicr fur le bord de voftre couche tout aurour; ces herbes leueront, \& fortiront hous de terre en fort pou de temps, \& firont arrachées toutes jeunes, de peur d'étouffer les plantes de melons, cela le.ur feruira de farciement \& labour tout enfemble.

Vous conurircz voltre couche tontes 1. snuits \& dansle mautrais temps, auce 
les peillallons qui porteront fur des traueife; d: bois, de la grofieur d'va cichalats, lefquelles feront foutenuës auec des fourchettes fichécs dans terre, au bord de la couche.

On lailfera domy picd ou peu moins d'épace entre les paillaffons \& la coucle, Ee en casqu'il luruint quelque gelée, ou naige, vous boucherez routel'efpaie qui fera cntre vofte couche ix les paillalions, auec du grand fumier fortant do defrous les cheurax, iufques à ce que le ma umais temps foit palfé.

Si voltre graine le boulcite par la trop grande chaleur qui lervit en la couche, ( ce que vous reconinoilfrez en peu de temps, car elle doit lcuer promptement) vous refimerez tout de notucau, en la rechaufrait par les coltés auec du fumicr bicu chaud.

Le viay temps pour femer les premicrs nalons, ett en la plaine Lune de Fevrier.

Ruand ils feront leuez, vous les couurirez atice des verres à boire, laillant quelque peu d'air entre la terre \& le vcrre, de peur gue le Mant s'eftualfiant ne iaunilfe.

Vous les lailiercz croifre jufgues à la quatrc ou hivienc feulles auan que de l.s replanter. 


\section{$100 \quad$ LEI I R D I N I R R}

L'on les replante en trois façons: La premiere fur dcscouches, que vous ferez à coltéde celle à efleucr le plant, \& toutes femblables: Vous ferez des trous dans le milieu de ces couches, de quatre en quatre pieds, \& dans chacun y meterez enuiron demy hoiffeau de terras, fans qu'il foit befoin d'en mettre par toute la couche, \& dans ce terras vous y planterez vos niclons, les ayant leuez de deffus voftre forme, auec la plus groffe motte que vous pourrez: Le foir à Soleil couchant \& apres, fera le vray temps que vous prendrez pour ce faire, \& s'il Ce peut que ce foit parvin beau jour, voftre plant s'en portera micux; vous luy ferez vn abry, à ce qu'il ne voye le Soleil de quatre ou cinq jours enfuiuans: vous les arrouferez les premiers jours a pres que vous les aurez replantez, afin cu'ils prennent Terre, \& s'accroiffent promptement.

Vous y metrez des cloches de verre, q:iy ieront jufques à ce que le Fruit foit deja gros \& autant de temps que la plante pourra tenir deffous la cloche laiffant toîjours vin peu d'air cntre la cloche \& la couche, de crainte d'étouffer la plante.

Depuis Les dix heures du matin, juf- 
ques à quatre heures aprés mydy vous pourrez leuer yos cloches de deffus vos melons, pour les fortifier contre le mauuais temps, \& vous les recoutrircz fur le foir.

Il arriue par fois des grefles qui carfent toutes les cloches, c'eft pourquoy l'on en a de faites auec du foarre pour couurir celles de verre, en cas que l'on voye venir quelque orage, \& pendant la nuit pour éuiter tel accident.

Quelques-vns font faire des cloche de Terre; mais je ne troute aucune raifnn en leur procedé : car le Soleil ne penet. tre pas cette Terre; comme il fait à trauers le verre, ils peuuent dire que c'ct pout la nuit feulement, \& contre la grelle, en ces cas là ils ont quelque raifon.

Vos melons fe fortifians, vous chofirés les principaux jets, (qui feront autant qu'il aura germè de grains) les au. tres rejets vous les châtrerez: \& quand vous verrez qu'il y aura trois ou quatre melons notiaz fur chaque jet, vousarrefterez fa trainalle, la rognant vin noud au defius de ccluy ou eft le Fruit; vous cfendrez fur voltre couche de colté \& d'autre les jets de la plante, pour à leuraife poumoir nourirle Fruit: quand 
IO2 LE IARDIN IER

il fera de la groffeur du poing, rous ne les arrouferez plus, fi ce n'elt dans vine excefinue feichereffe, quand vous verrez les fueilles fe brûler, \& la plante s'èchauder : en ce cas rous donnerez quelque peu d'eau à chaque picd languillant.

Vous mettrés des tiluilleaux deffous vos melons, afin qu'ils fe façonnent, \& auancent de meurir par la reflexion que le Soleil fait fur la thuile, ce qu'il ne feroir pas fur le fumier, ontre ce ils feront plus feichement, \& participe ront moins de la mauuaife qualité du fumier.

Vous ne fouffrirez qu aucun petit jet nouneau, tirela Scue de la plante, mais vous le rognerez, fi ce n'eft que voltre Fruit fut trop découncrt, \& qu'il eût befoin de quelque fueille pour fauorifer fon accroiffement, de crainte qu'il ne séchaude.

La feconde methode de tranfplanter les melons, eft de faire dés la fin de l'Efté des folfez de deux pieds de haut, fur quatre de large ( les Tourangeaux les appellent des Anjoux) \& laiffer vne efpace de trois pieds entre deux pour jetrer les terres, que vous accommoderez en dos d'Afne; puis dansle folfé vous y 
mettrés de bon fumier, terrier bien pourry, \&z les curures des follés, tirées de deux ou trois ans, \& meuries par les pluyes \&r gelées.

Au mois de Mars, l'Hyuer ayant bien meury le tout, vous ferés befcher, \& bien mefler la terre qu'aurés mis en ados, auec les fumiers de follé, y adiountant de nouveaux fumiers bien pourris, \& remplirez entierement voltre folfé : Vous empeicherés aufil l'herbe d'y venir, iufques à ce qu'en la faifon vous y replantiez les Melons, en la maniere que ic vous viens de dire.

Il) a vne troifiefme façon bien plus fa. cile, dont ie me troume auff bien quo des deux premiers, 2 ìy tous les ans. eu des Melons en quantité, \& de haute feue ( mais i'en atribue la principale caufeau fonds de mon Iardin, qui eft vin fablon amendédés long-temps) On n'y fait autre chofe, finon de donner deux ou trois labours à la terre, deuant\&apres l'hyuer; \& au tempsde replanter, $\mathrm{O}_{n}$ fait des trous au milieu des planches, dans lefquels on met a chacun vn boiffeau de petit fumier de vieille couche, \& dedans on plante comme ie vous ay enfeigné cy-deuant.

Si pendant les exceffiues chaleurs, vous E iiij 
104 LE IARDINIER

voy ez que vos $M r$ ! ons patiflent far trop de foif, \& s'efchaudent (comme l'ou dit) il ferabon deleur donner à chacun pied $\nabla n$ arroufoir d'eau, mais que ce ne foit qu'à l'extreme neccffité, \& rarement.

Pour fçauoir quand voltre Melon fera bon à cueillir, vous reconnoiftrés fa ma turité quand la queuci femble fe vouloir détacher du fruit, quand ils commencentà iaunir par deffous : quand le petit Iet qui eft au mefme noud fi deffeiche: \& quand en les flairant vous y trounercz de l'Odeur : maisceux qui ont confume d'eftre dans les melonnicres, le ingent aे l'ail, remarquans vin chiangement de couleur au fruit, quile tefmoigne eftre en fa maturité par vne iauniffe qui eft entre les coftes.

Les melons qui s'nuuragent ou bordent, font ordinairement douze ouguinze iours à fe façonner, atiant que d'eAtre meurs-

Pour la cueillette, elle fe fera amefure qu'ils fe tournent: fi c'eft pour envoyer au loin, yous les cueillerés dés l'inftant qu'ils commenceront à tournner, car ils s acheueront de meurir par le chemin: Si ce n'eft pour manger promptement, vous les cueillerés bien meurs, les mettant dans vn feau d'eau fraicfche tirée du 


\section{F}

ruys, \& les laifferés rafraichir comme l'on fait le vin, à caufe qu'en fortant de la melonniere ils font efchauflez du Soleil, \& feroient defagreables à manger, les autres que cueillirez à mefure qu'ils meuriront, feront pofeés fur quelque aix en lieu frais, \&zeruis felon leur maruritế.

Vous lailferés à chaque melon le noud qui tient à la queuë, auec quelques deux ou trois feuilles pour l'ornement, \& vous garderés bien d'arracher la quenë: car le melon s'euenteroit (comme fait vn muid de vin debondonné) perdant la bonté de ra reue.

Jl faut s'affuiettir à vifiter la melonniere au moins quatre fois le iour, au temps que les melons meuriffent autre ment ils fe pafferoient de meurir, \& ne feroient ff fains, mais mollalfes \& pleins d'eat.

Pour choifir vn bon melon, il faut qu' il ne foit ne trop verd ne trop meur; qu'il foit bien nourry, ayant la queuë grof-

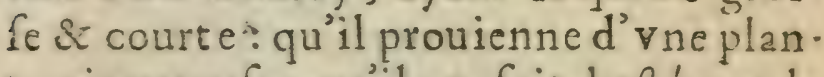
te vigourcufe: qquil ne foit hafté par la trop grande chaleur : qu'il foit pefent à la main, ferme en le preffant \& non mo. laffe; fec \& vermeil par dedans.

So uuenez-vous de referuer les Graines de c eux que vous aurés troulucz bons, \& 
106 IE IAR D I N IER

les plushat ifs; comme ie vous ay aducrty cy-deuant) \& les conferuez bien, elle eft meille ure de deux ou trois ans que d'vn.

Les Concombres fe fement \& efleuent furla mefme Couche, \& en mefme temps que les melons : ayant auparauant fait tremper la graine, dans de bon lait de Vache ou de Femme; il y en a de blancs $\&$ de verds que l'on nomme Perroquets: C'eft pourquoy dés vos premiers fruits vous vous abftiendrez d'en cueillir quelques-vns des plus beaux, des plus blanes, \& es plus longs pour en tirer la graine, que vous laifferés meurir fur le pied, iufques à la fin de la plante, gui fcra aux premieres gelées: Car quant aux Perroquets, vous les fereés tous manger: $d^{\prime} 2 u-$ tant que la graine des blancs dégenere fifés en Perroquets.

L'on les tranfplante aufi comme les Melons, fur couche \& en plaine terre: ils veulent eftre beaucoup arroufés pour leur faire pouffer quantité de fruit: On les chaftrera de jets fuperflus, \& de ceux qui n'ont que de faulces fleurs qui ne moiient point a fruit.

Les premieres fraicheurs leur font venirle Meufnier, qui eft que les fueilIcs fe farinent \& blanchiffent, cela tef- 


\section{FRA N ÇOIs.}

moignert qu'ils font proches de leur fin.

Pour la cueillette, elle ne fe fera qu'à melure que vous en aurés affaire, grolfiflans toujours, mais auffi sendurciffans, \&la graine fe formant dedans les rend plus defagreables au manger, leur vraye bonté clt auparauant qu ils commencent à jaunir.

Les Citrouilles s'efleuent auffi fur la Couche, fe replantent comme les Melons \& Concombres, mais plus communément en plaine terre : vous les placerés en quelque endroit de voltre Iardin alfez fpacicux, à caufe de leur Iets qui fe trainent par fois affés loin fans noüer da fruit.

Pour les replanter, vous ferés des trous forts enoignés les vns des autres, comme de deux thoifes, \& mettrez à chacun deux boiffeaux de petit fumier, à caufe de la force de la plante, \& les ferez bien arroufer.

Leur cueillette eft quand elles font en parfaite maturité, que l'on appelle Aouftées: elles ne fe gaftent point fur la tcr$r e$, mais fe meuriffent de plus en plus. Aux premieres fraifcheurs, l'on les cueillera les matins, les mettant elliuyer en monceau à la chaleur du iour : puss on les ferrera en lieu temperé du chaud

$$
\text { E }
$$


\&zdufroid, fur desaix lans fe toucher: \& furtout on les gardera de la gelée, car elles feroient entierement perduès.

Si vous en auez partrop, ou plus qu'if $n$ 'en eft de befoin pour nourrir voltre fa. mille : vous en mettrez dans le pain du commun, mefmenent dans le voltre: Pource faire, vous ferés boüillir de la Citrouiille tout de la mefme façon que celle que l'onfricalic, pourtant vin pers plus cuitte: puis vous cifoutcrés l'eau; \& detremperés voltre farine auec cette Citroüille, \& en ferez le jain à l'ordinaire : il fera plus iaune, de meillcur gouf, vn peu gras cuit, Ertres fain pour ceux qui ont befoin de rafraichifiemens.

Les Potirons blancs \& colores, Bonnets de Preftres, Trompettes d'Efpagne, Courges, \& fruits femblables, ne demandent autre gouternoment que leś Citroiilles; finon, qua acuns veulent eftre ramés \& non rampans deflus la terre.

Quant à leurgraine: comme auff celle des Citroiiilles : elle ne fe recucille qu'̀े mefure que l'on dépece les fruits pour eftremargets : \& il la faut bien effuyer à l'air a puis la bien enfermer de crainte des Souris, qui la mangent comme celle des. anclons \& Concombres. 


\section{FRA N ÇOI:}

Des Artibaur, Cordons \&े Aperges.

\section{SECIION II.}

T Artichaud eft vn des excellens fruits de tout le Iardin Potager, \& recommandable non feulement à caule de fa bonté, \& diuers aprets quel'on luy donne; mais encore pource que l'on prend long temps du fruit fur fa plante.

Il y en a de deux fortes, de Violets \& de Verds: les Oeilletons qui font, à colté des vieilles Souches feruent de Plant, lequel veut eftre logé en bonne terre bien effondréc, fuméc, \& labourée de deur on trois labours.

Les geltées eftant enticrement palfées: au mois de May l'on plantera ces aille. tons, les ayant feparez de leur Mere, auec le pluade racines que l'on pourra, à caufe de la facilité de la reprife, \& s'ils fontforts, ils porteront des Pommes dés l'Automne enfuiuant.

Vous les planterés à quatrc ou cinq pieds l'vn de l'autre, felon la bonté de la terre; car fi elle eft fabloneufe \& legere, il les fauda planter plus dru fi elle eft forte, d'autant que les fueilles en viendront plus grandes, le fruit plus gros, 
\& qu'ils ietteront plus de regrains; audi faudra-il qu'ils foient plantez à plus grande diftance.

Ils ne veulcnt autre culture auaut $l^{\prime} h y$. ver que d'eftre labourez par fois, pour empefcher l'herbed'y venir.

L'Hyver, vous les coumirez pour les preferuer de la gcléc: pour ce faire, l'on s'y gounerne de diuerícs façons: L es vns apres auoir coupé toutes les plantes à vn pied pres de terre, \& rallemblé ce qui refte des fueilles, (comme l'on fait la Chicorée pour la blanchir ́ fe contentent de lesbuter en forme de groule I aulpiniere; lailiant paffer le haut des fueil. les deux doigts feulement pour ne pas eftouffer la plante, \& mettent par dellus du grandfumier, les preferuant ainide gelée, \& que l'cau des pluyes n'entre dedans.

Les autres font des rayons entre deux rangées, \& iettent la tcrre en frrme de dos d'afne fur les Plantes, les courunt à deux doigts pres, cóme ic viens de dire.

Et lesantres n'y mettent que de grands fumiers autour de chaque plante, \& ils ne laifent pas de leur faire palfer I'hyuer fans peril : toutes ces trois façons font fort bonnes, chacune ayant $f_{a}$ raifon pourquoy. 
Vous les butterez le plus tard que vous pourrez, de crainte quils ne pourriffent, mais auffi prenez garde que quelques fortes gelées ne vous furprennent, fi vous en auez beaucoup à gounerner.

Si vous voulez auoir du fruit dans l'Automne, il ne faudra que couper la tige des premiers qui auront porté du fruit au printemps, pour l'empefcher qu'elle ne iette desregains: \& dans l'Automne, ces forts picds ne manqueront de ietter de belles Pommes, pourueu que vous les entreteniez bien de labour, \&z les arroufiez en leur neceffité : \& auffi que vous leur oftiez les cilletons des coftez, qui tireroient toute la fubftance de la plante.

L'hyuer eftant palfé, vous découurirez vos artichaux non pas tout d'rn coup, mais peu à peu, de crainte que les fraifcheurs ne les gaftent, à caufe de la tendreffe qu'ils ont ayant efté eftouffez dans la terre: il faudra so y mettre à trois reprifes de quatre en quatre iours : au bout defquels vous les labourerez, \& defchaufferez bien, les defchargeant de plufieurs petits œilletons, \& n’en lailfant à chaque pied que trois des plus forts, pour porter le fruit.

Pour tirer des Cardes d'Artichaux, vous vous feruirez des vieils pieds que 
I12 LE IARDINIER

voulez ruiner: car il eft bon de cinq en cinqans; de planter vn nouucau quarré d'Arcichaux : àcaufe que la plante a effritté la terre, \& ne produit plus que de petites pommes

Les premiers fruits eftans cueillis, vous rognerez les plantes à demy pied pres de terre, \& coupercz la Tige le plus bas que vous pourrez, les æillentons poufferont de tres grande force, \& eftans à trois pieds de haut ou enuiron, vous les lierez auec du grand foarre, fans les ferrer beaucoup, puis les entourerez de grand fumier, cela les fera blanchir.

Vous les pourrez laiffer iufques aux grandes gelées que vous les cucillercz \& ferrerez dedans la Caue, ou autre lieu exempt du froid.

Vous en cueillerez de iour à autre, qu'en aurez de befoin, commençant par les plus grandes, s: lailfant les autres qui s'enforciront en peu de temps, ayant tonte la nourriture de la plante.

- Quant aux Cardons d'Épagne, ils ne font fi delicats à goumerner que ceus d'Artichaux : aufi ne produifent-ils des Cardes fi douces, ny fi tendres: on les we de la mefme façon que ceux d'Artichaux pour les faire blanchir.

Ils fe fement de graine \& fe replantent auff d'œilletons. 
Les fleurs des Cardons, qui font de petites barbes violettes feichées à l'air, leruent à faire prondre le Laict, \& le caillent comme fait la prefure: Les Efpagnols \& les Languedochiens en vfent 2infi.

Les Afferges fe fement de graine fur vne planchè à part, dont la terre aura efté preparése par plufieurs labours, \&5 bicnfumée: à deux anson les leve $\&$ on les tranfplante.

Pour les loger, il faut faire des forfez de guatre en quatre pieds, \& de dcux de profondeur, les bien aquario par le forids, \& ietter la rerre efgalíement des deux coftez, I accommodant en dos d'Alne, puis donner vn bon labunr au fond de vosfollez, y mellant dit petic fumier, se drelfer ce labour bien vny par tout : par apres vous placerez vos plantes d'Afparges au cordeau à trois pieds l'vne de l'autre, metrant deux plantes cufembles, vous les placerez tout au bord de chaque colté de voltre folfé, \& ce dautant que labourant les fentiers, \& par fucceffion les reduifant à pied \& demy de large, la terre fe icttera fur la planche: alors vous pourrez labourer plus d'vn bon pied de lirge de cliaque coftéde vos Afperges, oul la terre 
eftoit efleuée, \&e par ainfi vos plantes iettcront quantité de racines du cofté des Sentiers.

Vous planterés vine troifiefme rangée d'Afparges au milieu de ces deux que ie viens de dire: Il fera bon de les mettre en franc de quarreau, afin que les Racines foient tant plus efloignées les rnes des autres, \& qu'clles seitendent par route voftre planche.

Quelques curieux mettent au fond de leurs tranchées des cornes de Mouton, \& tiennent comme pour vne chofe affeu. rée. qu'elles ont vne fympathic auec les Afperges, quicarfe qu'elles en profitent mieux, ie m'en raporte à ce qu'ils en ont experimenté.

Pour les labours, il ne s'en donne que trois parchacunan: Le premier quand les Afpergescelfent de poulfer : Le fecond, à l'entrée de l'hyuer: Et le troifiefme, yn peu auparauant que les $A f$ perges commencent à poufler: A chaque labour vous remplirez, ou rehaufferez voftre planche d'enuiron quatre doigts, y icttant la terre de vos Sentiers: \& par delfus vous y ferez mettre enuiron deux doigts de haut de fumier de vieille Couche.

Vous ferés trois ansou plus fans con- 


\section{FRA N Ç. OIs.}

per aucune Aiperge, afin que la plante fefortifie, \& ne sallouchilfe point, antrement vous nenariez que de petites.

$S i$ vous eftes iufques à quatre ou cing ans fans en couper; vous en cueillerés d'aufin grofles que de gros Poireaux, apres lequel temps paffe, vous cin couperés inceifamment, lailfant les plus petites monter à graine, a fin que la plante fe fortifie d'autant plus.

Pendant les quatre années que vous ne couperés aucune Afperge, obieruant à chaque labour ce que ie vous ay dit: voltre plan he fe remplira, \& vos Sentiers eftans defchargez, vous les pourrés labourer, y mettans deflous le habour, de bon fumier bien gras.

Vous fçaurezque la plante d'Afperge monte toûjours, \& gu apres que ha terre de vos Sentiers aura efté iettée delfus les planchers, il fera neceifaire d'y rapporter des terres (les dreffant fur la place en forme de coutucrcle de Bahut) autrement elles feroient découmertes, \& periroient.

Pour cucillir vos Afperges, il faudra ofter vn peu de terre d'antour celles que voudrés cueillir, de crainte d'en couper d'autres qui pouflent : puis veus les couperés le plus bas que vous pourés, \& prendrés garde d'offenfer celles qui font 
II6 $L E I A R D I N I E R$

cachés, car ce feroit autant de perte, \& ne feroit qu'aliouchir les plantes.

A celles que vous verrés qui n'en produifent que de petites, vous n'y couperés rien, afin qu'elles fe fortifient : comme auffi vous laifferés monter à graine, celles qui peuuent à chaque planche fur la fin de leur faifon: cela reftaure entierement les plantes de toutes les playes que vous leur auez faites, en les defpoiillant de leur fruit.

Des C'Boux ơ des La at::ës de toutes ejpeces.

\section{SECTION III.}

TL y a de tant d'effeces de Choux, que difficilement volis vous refoudrés à cn auoir de toutes en voltre Iardin, car ils occuperoient vne trop grande partie de voftre terre: ćeft pourquoy vous choifrés ceux qui fernnt les plus agreables ì voftre gouft, \& qui viendront les plus tendres, \& les plus faciles à cuires : à caufe que la terre qui les produit, \& l'eau quiles cuit, les rendent ou meilleurs, ou moindres en bonté.

L'on nous apporte des graines d'Italie, \& nous en auons aufil de France: celles d'Italic font les Choux Fleurs, les 
Romains, de Veronne, de Milan, les Choux, Raues, les Pancaliers, ceux de Gennes, les Frifez \& autres.

Dz France, nous anons les pommez de plufieurs fortes, \& ceus qui ne pomment point : c'elt pourguny je troune fort à propos de traitter icy de chaque forte à part, le plus fuccintement qu'il me fera poffible.

Les Choux-fleurs comme les plus precieux de tous, feront mis icy les premiers: La graine nous eft apportée d'I talie, Ex les Italiens la réçoiuent de l'Iile de Candie \&: autres cndroits du Levant: ce n'eft pas que l'on rícn recueille d'a'fez bonne en Italic \& cn France aufít: mais elle ne produit pas de in groffes pommes, ou tefles, \& et rujecte a dicgenerer en Choux, Ranes, \& Nanets: partant, il fera bon de sen foumir de la meillenrede Leuant, ou par amy, ou par quelque alleurée indelligence à Rome : Los Marchands Lingers, \& Gantiers de $\mathrm{Pa}$ ris, vous pourront donner du contentement en voftre affaire; car ils y trafiquent de leur Linge, Pallemens, \& Gands.

Pour connoiftre la bonne graine ' gui eft la plus nounelle) il faut que eile thit d've couleur viue, fort pleine d'huile, 


\section{I I I A R D I N I ER}

bicn ronde, \& nouridée petice, ou deífeichée, ce quila témoigneroit eltre vieil. le, qu'elle foit de couleur brune, \& non pas d'vn rouge clair, qui feroit paroiftre qu'elle n'auroit pas bien meury fur le pied.

Eltant pourueu de boune graine, vous la femerez ou à la maniere d'Italie, ou de France : Pour la goumerner à l'Iraliemne, on la femedans desquailies, ou bacquers durant la pleine Lune d'Aouft; clle leue promptement de Terre, \& fe fortifie anant l'Hyuer : La gelée venante, vous mettrez vos baccuets dans la cale, ou dans la ferre, juiques au renouncau, quelesgelécs chant toutespaiLées, vous les replanterez en bonne terre: \& par ainfi vous aurez des pommes belles, blanches, \& bien conditionnées, a.l1parauant les grandes chaleurs.

Les Italiens n'attendent pas que les rommes foient toutes en leur groffeur, mais peu auparanant ils les arrachent, \& les mettentà la caule, enterrant toute la racine, \& la tig a jufques à la pomme, les rangeant cofte à cofte vin peu en penchant : là ils s'achouent de pommer, \& fe gardent fort long-temps, au licu que sil: les lairfoient fur terre, la chaleur les ferwit monter à graine. 


\section{FRA ÇOIs.}

Les François fe contentent d'en auoir pour la find Automne, qu'ils conferuent pour manger durant l'Hyuer : ce n'elt pas que quand ils font faits de bonne heure, qu'il n'y en ait quelques-vns qui ne faffent leurs pommes vers le mois de Iuillet: mais les zutres s'endurcitfent par les grandes chaleurs \& demeurent fans profiter faute d'humidité, ne produifansque de petites teftes, le plus ioutient point: C'elt pourquoy vous en femerez peu fur voltre premiere couche dans voAtremelonaicre, en faifant de petits lillonsà quatre doigts l'vn de l'autre, dans lefqucls vous épandrez voltre graine, affez claire pour éuiter la confufion, \& la recounrirés de fon terras: deux ou trois fillons feront plus que fuffians pour yous en fournir amplement.

Vers la fin d'Auril, quand vos Melons feront hurs de deffus la couche, $\&$ : replantez, vous pourrez y refemer d'autres Choux-fleurs, (de la façon que je viens de dire ) lefquels feront leurs pommesdans l'Automne, \& feront ferrezen lieu exempt de la gelée, pour eftremangez pendant l'Hyuer.

Pour les replanter, vous attendrez qu'ils ayent la fueille large comme la paulme de la main, afin qu'ils loient 
Iio IEIARIINIER

plus forts; vous leur rognerez le bout $\$$ : $: 1$ piuot, \& les enterrerez jufques au collet, c'eft à dire, que les fueilles de la cime ne refortent que de trois doiges hors de terre; ou bien pourm expliquer plus clairement, vous les enterrerez juiques au dernier noud d'enhaut, encor faudra- $t$ il que vous faffiez dans la terre comme de perits baffins de demy pied de diamettre, \& quatre doigts de profond cur, afin que l'ean aille direct ment au pied quand rous les arroulcrez, eftant comme inutileau refte de la terre, oi il n'y a aucunc racine.

La vraye difance pour les replanter. eft de trois en trois picds, \& deux rangs reulement à chaque planche: On foignera à les farcler \& labourer quand ils cn auront befoin, jufques à ce que les fueil.. les couurent la terre, \& eftouftent l'herbe qui pourroit pouffer.

Si vous voulez faire des trous à $l^{3} \mathrm{en}$ droit où vous les planterez, iz mefler de petit fumier comme j'ay ditaux melons, \& concombres, wous en aurez plus de contentement, car ils feront la pomme de beaucoup plus grolle.

Tous choux, mefmement toute plante telle quelle foit, veut eftre arronféc foigncufement les premiers jours qu'ils 
Cont replantez, pour leur aider à la reprife: ce que vous reconnoiftrez eftre, quand les fueilles fe fouftiennent d'elles melmes, \& ne fe laiffent point aller fur terre.

Les Choux de toutes les efpeces, fe femeront fur la couche des Melons, pendant qu'elle a encor de la chaleur, afin qu'elle germe \& leue promptement de terre : il faut faire de petits rayons en trauers de voftre couche, \& y femer la - graine affez claire \& non confuie.

Dansle mois d'Auril, vous en refemerez d'autre fur la mefme couche, ì la place, d'où vous aubez leué vos melons, \& concombres.

Or d'autant que les Oyfeaux font fort ấpres à manger la graine lors qu' elle leuie de terre à caufe quelle porte fon écorce au bout de fa fueille: je vous veux enfeigner des moyens pour les eitranger: l'vn, en mettant, quelque rets par deffus la couche, qui foit fouftenuè à demy pied de haut: l'autre, en faifant de pecits moulinets de carte, comme ceur dont les enfans fe joüint en courant contre le vent: \& l'autre, en faifantaulieu de carte auec du bois de Sapin leger, comme de boëttes à confitures : \& à $\Gamma^{\prime} \mathrm{Ar}$. bre qui foufticndra les ailles, ny mettre 
$1 \approx 2$ LE I S.R D I IV I E IR

Cuelque grelot, cela empeichera de jeur lis Oyfeaux, \& de nuit les Mulots:car jour peu de rent quali falie, ilscourneront \& fatisferont à vofre intention.

Daus les couches il s'ungendre des orbecs, \& des courtillieres, qui rongent les fimences, \& lesgermes pour les pren$\dot{d i c} \mathrm{c}$ il faut enterrer daus la couche quelques petits vailfeaux, commedes verres à boire, \& antres: il les falit enfoncer rois doigts plus bas quic le haut de la cou. che : les empliliant d'cau à deux doigis frés du bord; ces vermines en conrant far la couche comberout dedans, \&- ie moyeront.

Les choux à large cofte ne feront icmez qu'au mois de May, à caúie de leur giande delicatelle. \& 5 ils ont aflez c'e Eurce pour eltre repinniez au commencer.cut du mois de Iuillet, ils pommusort d.uanel'Hyur, à mongocitiln'y a poine d'efpece de choux gui egant cenx-lì, cor is cuifent prompecine, \& font in dilicits, que lis plus gros cottons fondent à la bouche: fi vous en mangez à jun va protage, où il y ait peu de pain,

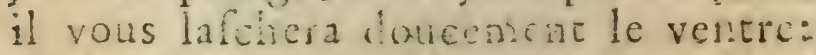
S. de plus, queli: quántió que vous en a jez nzarce, clla ne vous renuoyera al-

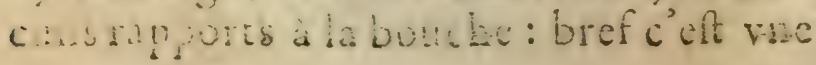


F r a n cor Is.

efpecc de (houx que je ne vous fçarois eroploüer, pour vous perfuader à en garnir voitre Iardin, pluitolt que de beaucoup d'autres:

Des Choux blancs pemmez, neus avons ceur de Flandre, qui viennent les pus gros \& defqucis la Porame élevéc en bonne Terre, pefura quaraure livies \& au doflus.

Ceur d'Aubervilliers, font extremement francs \&idelicats au manger.

Il y a une autre force de Choux qui font foüertez de quel ues veines rouges, \& dont le pied au tempsque l'on icsrepiante eft tout pourpré; ccix là rie icmblent les plus francs de tous, car ils pomment tout pres de Terre, jettent peu de Fuisi? le avant que de pommer, \& le ferrad relle façun, queia Pomme eft touce piatte par delius.

Les Choux rouges aurone aufi: quelque petice place dians volire Iarcilin, pour la necefiré en cortaines naladies.

Il y a encore d'une autre cfrece de Choux extremement muiquez qui ne font qu'une petite Pomme, mais ils font beaucoup à prifer à caule de leur bonne odeur.

Les Choux Tendres ou Plonds, ne fe fementquaux mis d'Asul, pcir citra 
I24 LEIARDINIER

replantez un peu avant l'Hyyer, ils s'accroiftront \& vous foumiront pendant tout l'Hyver, particulierement les jours des fortes Gelées, quiles attendriffent $\&$ les rendent tres agreables au manger.

L'on replante aufit de toutes les efpeces de Choux d'Italic, \& les Pancaliers font les plus en eftime, à caufe de leur bon gouft parfumé.

Pour replanter toutes fortes de Choux. 1a Terre eftant profondement labouríe, \& bien fuméc fousle labour, vous formerez des Planches de quatre pieds de large: \& à un pied prés dú bord, vous ferez un petit rayon de quatre doigts de profondeur, large de demy picd, revenant à rien paren bas, comme une raye de Terreà Bled labourée de nouvcau: dans ce rayon, vers la fin du jour, en beau temps, vous ferez des trous avec lc Plantoir, \& plantercz vos Choux jufques au Collet des plustcndres Fueilles, en rognant le bout du pivot: Vous les cfpacerez à diftance convenable felon qu'ils grandiffent \& eftalent, puis les ferez arroufer foigneufement, refpandant l'Eau dans cette Rigole feulement d'autant qu'il n'eft necclfaire d'arroufer toute la plante.

L'on pourra auff Ios replanterez en 
confufion dans des quarrez entiers, particulierement les Blons pour geler; mais celan'ett fi commode que par planches pour l'arroufement: joint auffi que chaque planche diftinguera plus facilement les efpeces que vous mettrez chacune à part.

Vous foignerezà ofter toutes les fueilles mortes des Choux pour plus de propreté ,évitant la mauvaife odeur qui provient de la corruption des Choux ; \& a fif que cettc pourriture accueille beaucoup de Vermine, comme Limats, Grenouillle, Crapaux, \& autres, qui ne font qu'endommager les Choux.

Quand vos pommes feront faites, fi vous en remarquez quelqu'vne qui vouluft monter à Graine : vous arracherez la plante à de $\mathrm{y}$, ou bien marcherez fur le Trone, failant pancher le Chou furle cofté; cela empefchera qu'il ne graine fi toft. \& vous donnera le temps d'eftre mangé en le prenant des premiers.

Pour la Graine, vous pourrez referver de vos plusbeaux Choux, en les replantant en quelcuues Abry des vents d'Hyver pendant les grandes Gelées, les couvrant a vec des pots de Terre, \& du grand Fumier par deffus : vous le découvrirez par fois dans le temps doux, \& quand il fera 
126 LE IARDIN TER

Solcil, pour les revigorer, citant foigneux de les revcir la nuict, peur d'eftre farpris de quelque gulée.

Vous en metterez d'autres dans la Serse, les pendant par la Racine en haut Guelques quinze jours : afin que toute l'Eau qui peut eftre entre les fueilles s'égoute, car elles pourriroient: lequel tems eitant paffé, vous les enterrerez jufques à la moití du Tronc, les metrant fi prés l'une de l'autre qu'ils fe puiffent toucher.

Pour les autres qui ne pominent point, il n'y a qu'à les replanter ou les laiffer en leur place, ils foufriront l'Hyver, \& graineront de bonne heurer

La Graine eftant meure, ce que vouts connoiftrez quand les premieres Gouffes feront feiches, \&z s'ouvriront d'elles-mémes: alors vous l'arkacherezdoncement, la cirant par Tige, \& l'accofterez à voftre Contre-Elpalier, pour la s'achever de meurir, \& feicher: Jl eft bon de l'y atracher avec quelque brin d'Ozier, de chainte gue le Vent la jettant par terre nen falfe efcouller beaucoup.

Dans le mois d'Aoult yous femerez des Choux à Pomme fur quelque Planche à part, pour la leur laiffer palfer l'Hyver en forme de Pepiniere, jufques au Rer.cuveau que vous les replanterez en la 
manicre quej'ay dite cy diuant: Sec'eit le moyen d'anoit des Pommes de trésbomne heure, diun eft foigneux da les bien gouneruer.

Il y a pluisurs petits animaux qui rongent \& endummagent les choux, tant en leur jeunelfe, qu'en leur accroillement, comme les ticquets, quilont vne petite elpecede mouche verie qui faure, lis limats, fourmis, puceons, St autres: le fenl remede que j’y troune, eft le frequent arroulicment quiles étrange, ou fait mourir : cardurant la grande fecherefle, on veit perir journellement les choux, par l'importunité de ces animaux.

Il fait bon femer des Choux à toutes les pleines Lunes des Mois, pendant tout le beau temps, afin de remedier aux defordres que ces petires beftioles vous font; vous de pourez faire facilement fans defpenis, cu yous feruant du labour c\%? lion anra domé à vos Elpaliers, où à efleuant vos jeunes Chons, les fraquents arroufement proficcront auffi à vas Aarbres.

Il y a d s houx curicux qui portent pluficurs Pommes lur vne mefme tige, mais ils ne Cont fi delicars que les antres.

Quand vous aurez coupé les teltes de yos Ciroux, fi vous ne voulez en arracher 
R. 8 LE IAR D INIER.

le trone, il vous repoulfera de petits reo jats, que les Italiens appellent Brocoli, Ex les François des Broques, elles fe mangent ordinairement en Carefme à la purée, \& en entremets, fur les meilleu. res tables.

Des Laictuës, il s'en trouue prefque auffi grande quantité que de Choux; $\mathrm{c}^{3} \mathrm{ct}$ pourquoy j'ay mis ces deux plantes en melme Chapitre.

Nous auons pour celles qui pom ment, les cabulles, celles à plufreurs teftes fur vit melme pied, celle à coquille, celles dic Gennes, la Romaine, \& la Frifée, gui pomme en forme de Chicoréc.

Les antres qui ne pomment point comme les Erifées fans pommer, \& beaucoup d'autres de flufentrs fortes.

Ee d'autrescncore qu'il faut lier pour Iss faire blanchir : qui font la Laictuë à feuille de Chefne, ou Royale, \&z les Chicons.

Elies fe fément pendant toute l'année, excepté l'Hyuer : car depuis que vous commencerez à en mettre fur voftre premiere couche ( ainfi que j'ay dit en l'artiele des Melons) iufques à la fin du mois d'Octobre vous en pourrez efleuer.

Pour les faire pommer il ne gift quà les replanter à demy pied, ou yn peu 
FRA NÇOIS。

plis l'vine de l'autre les planches des Efpaliers, \& Contr'Épaliers, y feront bien proprcs, fans occuper aucune autre partie de voftre Iardin: durant les grandes chaleurs elle auront peine à pommer, fi ce n'eft à force d'arroufemens, la faifon les faifant monter à graine.

Celles de Gennes font à preferer à toutes les autres, à caufe de leur grolfeur, \& qu'elles palfent l'Hyzer fur Terre eftant replantées, où vous en pourez prendre pour les Pottages, \& auffi qu'elles vous donnent les Pommes, dés la fin d'Auril.

Pour celles qui ne pomment point il n'y a qu'à les femer, \& à mefure qu'elles croiftront les efclaircir ( c'eft à dire ofter le trop) afin de donner lieu à celles qui refteront de s'élargir \& augmenter: aucuns les replantent, mais c'eft fe donner trop de peine, pour vne plante fi facile à efleuer.

Les Royales, \& Chicons fe veulent replanter à vn pied ou plus éloignéee les vnes des autres: \& quand vous verrez que leurs plantes courriront toute leur Terre, alors par vn beau temps, non pluvieux, \& la rozée du matin eftant effuyce, vous les lierez de deux ou trois liens par étages, auec du foarre long; \& 
I30. LE IATD INIER

ce à plulieurs reprifis (c'elt à dire) en ne las liant pas tontes comme elles fe rencontrent fous la main, mai, choififant les plus fortes les premieres, pour donner de l'air aux foibles, cela fera auff gu'elles vous dureront plus long-temps, les premieres eftans blanches, auparauant que les dernieres foient liées.

Si vous defirez les faire blanchir bicn promptement, volis metircz par delfus chaque plante vis pot deterre, femblable aux creuzers des Orfevres, \& les couurirez de fumier bien chaud, ccla les fera blanchir en fort peude temps.

Pour la graine de Laict 1 e de toute les fortes, elle eft fort facile à recueillir, à caufe que les grandes chaleurs en fout monter beaucoup plus que l'on ne vouidroit de celles qui auroient efté femées les premieres : vous les atiacherez quand vousverrés, qu'il y aura plus de la moitié des fieurs paffée, \& les accoufterez tout debout contre les lattes devos Contr'Erpaliers, les laillant meurir, \& deffeicher dix ou douze jours: puis eftant bien feiche vous la froifferez entre vos mains, la nettoyerez de la balfe, \& la ferrerez mettont chaque efpece à part. 
Lies Racines.

\section{SECTION IV.}

Y. Es Berte raues, commeles plus grof1. Ces leront mifes en tefte de ce Chapitre: élles veulent eftre placées en bonne Terre, bien fumée, \&r bien effondrée, pour produire leurs racincs bicn longues, grolles, \&i non fourchuës : carli elles ne trouben le fonds de la Terre à leur goult, clles s'élargiront affcz, \& grofilsont par la tefte, mais dansle milieu il s'y fait vn trou qui profonde bien auant, Iss rendant dures \& pleines de filets, perdant beaucoup de leur coul cur rouge, qui fait que l'on les méprife: C'eft fourquoy in vous ne voulez faire effondrer voltrelardin, craignant la dépenfe, vous ferez au moins befeher deux labours l'vnfurl'autre, en la maniere que ie vous vais enfeigner, qui n'eft qu'vndiminutif de l'effondrement.

Il faudrabefcher vne Orne toute de la lougueur du carré, d'vn bon pied de profondeur, \& de deux pieds de large, e: jectant la Terre tout d'vn colté puis be sher va fecond labour dans ce melme folsé. le plus profond que l'on pourra fans 
I3:2 LE I A R D I N I E R

en fortir la Terre : par aprés y mettre enuiron quatre doigts de haut de bon fumier bien gras, comme ccluy qu'on tire de delfous les Vaches \& Moutons, pendantque l'on ne les affourre point; puis befcher vne feconde Orne, jettant la premiere litiere fur ce fumier: parapres befcher la feconde litiere, \& fur ce labour mettre du fumier, comme j'ay dit cydelfus, \&r en continuant de la mefme facon, jufques au bout de voltre carré: La derniere Orne demeurcra balle d'vne litiere, à quoy vous auiferez de trois moyens lequel vous agréra le plus, \& qui vous fera à moindre dépenfe pour la remplir, ou d'y faire porter les Terres, qu'aureztirées de la premiere liticre, de la premiere Orne: ou de les faire rattirer pour mettre voftre carré de Niueau; ou de lailfer ce folfé vuide, pour y jetter toutes les farclures du Iardin, afin que là elles fe confomment en fumier, referwant à rendre voftre haire égale, à mefure. que les labours fe donneront.

Cette façon de ménagement, eft-ce que je vous ay voulu dire cy-deuant, à la premiere Section du premier Traité quand j'ay parlé de l'effondrement de la Terre, oi j'ay promis vne maniere pour fleuer toutes fortes d'herbes potageres legumes. 
L'Hyuer eftant entierement paffé, vous fenerez vos Bettes - raues fur quelque bande de vos Efpaliers : puis eftant alfez fortes, vous les replanterez par planches, les mettant à vn pied prés l'vne de l'autre, pour la facilité de leur accroiffement, rejettant celles que vous trouuerez fourchuës, comme de nulle valeur: vous ne negligerez de les biner, \& farcler curieufement de toute herbe, ce faifant vous en recueillirez de trés belles, pourueu que vous ne les laiffiez patir de foif pendant la grande chaleur.

Quelques-vns neles replantent point, mais auffi elles ne vicnnent pas fi belles, \& font fujettes à eftre fourchuës, pour lesreplanter, vous obferucrez la mefme chofe que j'ay dite aux Choux, referué qu'il ne faut pas leur rogner le piuot.

Vn peu auparauant les gclées, vous les tirerez de Terre, \& les mettrez dans la ferre, enterrant toute Ia racine dans du fablon jufques au collet, les jonchant colteà colte, vin peu en penchant, \& jettant vn lit de fablon par deffus; puis rerrettrez vn autre lit de Bette-raues, puis $1 \mathrm{n}$ lit de fablon, \& en continuant jufques à la derniere, elles s'y conferueront trés-bien, les prenant là à yoftre befoin toutes de rang, lans en tirer du 
$13: 4$

\section{E IAR I N IER}

mi.ien ou des denx coltez na élchione

Pour ha rraine, yous referuerez des plas belles iacines, $\&$ des pius longues, gue vous enterrercz comme les aures, F." au Printemps los planter en guele que cutroit vuide devolera Contrifpalier; d'autant gue vous y pourrez arre. fer lon montant, que les vents renuerferoient à caule de fatrop grande hauteur, \& charge, s'i! n'utcit foultenu: fi vous n'aymez micux les metrant cn queique planche les appuyer aucedes pieux bien forts. Lagraine flant meure, vous arracherezles plante \& les lierczà vos Contre Elpaliers, afin qu'illes śacheucucnt de meurir, \& Ceichent plusfacilement.

Les Carrotes, \& Panets fe gouturnem comme la Bette-rane, ils ne Iont fi ten. dres au froid paflans allément l'Hyuer dans la Terre fans fe galter jufanes au renuulua qu'ils montert en graine, \& ne valent plusrien à manger, vous entio rerez de Terre pour voltre pronilion pen * dant l'Hyuer, lefquels yous ferrerez commeles. Bettes-raues.

Il y a des Carroces de deux couleurs, de jaunes, ix de rouges: les jaun ij font les plus delicatesà metre au por. ou autrement: fi vous voulez en manger de tendres au mais de May, comme les 
Picards à Amiens quiles mettent au poe en guile d'herbes ( il facidra fumer la Terre, \& la preparer par bons labours durant l'Enté : au mois d'itount vous les femerez la Lune eftante en fon decours, elles leueront auane l'Hyure, \& en farclant les maunaifes herbes, vousles eiclaircirés cù vous y verrés de la confulion, d'autant qu'il neft tefoin de les tranfplanter comme les Bettes raues.

Pour la graine, vouschoilirezdes plas belles, \& des blus longtes, que ferrerezen la caue pendant l'Hyuer, \& los remetrez on Terre au renouleau comme les Deites-rauts, pour les laiffer monter à graine; fi vous en laiffez quelque-vnes dans Ierre, elles y pafieront ailément THyuer fans fe pourri, \& monterontà Graine en fa fation: mais il elt mieux de lestirer hors da Tcire, ain que j'ay dit: d'autant que vous choifirez les plus belles, les plus grof́les, \&z les plu, longues pour engeance: ce que vous obleruerez auffien toutes fortes de plantes, fi vous voulez eAtre toujjours fourny du meilleur.

Les Salifix qui fe cultiuent dans les Tardins font de deux efpeces : il yen a qui fleuriflent violets, te font les communs, \& d'autres jaunes, ceux-cy font les $S_{a} l$ fifix d'Efpagne, que lion nornme Scor- 
136 LE I A R D I N I E R

fonere, ils font differens de fuieillage, auni bien que de fleur : car les violets ont la fuieille femblable au petit Plantin à cinq nerfs, \& les jaunes l'ont beaucoup plus large.

Il y a fort peu de temps que nous avons cette Scorfonere en France, $\&$ je crois en avoir eu des premieres: c'eft une racine beaucoup plus delicateau manger que le Salfifix commun \& elle a cét avantage par deffus toutes les autres Racines, qu'elle $n$ a fe pace point dans Terre comme les autres qui fe cordent, \& ne durent que une année, laiflez l'y tant que vous voudrés, elle groffira toûjours, \&ífera en tout temps prefte à manger, quov qu'elle monte à Graine tous les ans: Il eft bon de ratifler fon Efcorce brunc (dont elle prend fon nom de Scorfonere) \& la faire un peu tremper dans l'Eau claire, a vant que de la mettre boüillir, à caufe qu'elle jette une petite amertume qu'elle retiendroit fans ccla, ce que ne font pas les Salfifix communs, qui eftand fimplement lavés, fe mettent boüillir, puis on leve la peau.

Ils fe fement en deux temps, au Renouveau, \& quand ils deffleuriffent, laiffans envoler leur Graine : pour plus grande propretè, \& commodité à les 


\section{Fĩ A N ÇO Is.}

Sarcler, on les feme en Rayon fur des Planches, quatre Rayons à chaque Planche : Quand ils monteront en Fleur, il faudra entourer la Planche avec des pieux, \& une Latte oul deux en forme de Contr'E Epalier, de crainte que les Vents ne les renverfent par Terre, au prejudice de la Graine: Les Communs fleuriffene pluftoft que crux d'Efpagne.

Pour recuillir la Graine, il faut quatre ou cing fois le jour vifiter vos Salfifix, car élle s'épanoüit comme un Piffenlict, \& s'envole: C'eft pourquoy l'on prandra garde auffi-toft qu'elle fora efpanoiiie, de ranalfer toutes les barbes, \& lestenant du bout des doigrs en arracher la Graine, que vous mettrés à mefure dans quelque pot de Terre, qui fera toüjours proche de la planche, pour efviter à la porter ferrer dans le logis à chaque vifite que vous y ferés, \& le couvrirés de quelque Tuile, de crainte qu'il ne pleuve dedans, \&que voftre graine ne foit mouiillés.

DeS R A VES, il $s^{\prime} e n$ trouve de trois épeces, LA R нев, он R A в в E ou gros RAIFORT, la RAVE-NoIRE\& le PETIT-RAIFORT, qui eft celle que l'on mange communément à Paris.

L A R A B B EOU R HEE, elt une viande groffiere fort commune en Limoges par- 


\section{IFS LE IARDINAER}

my les pauvre Gens quien font plufieurs fortes eie Mets, bouillis. fricallés \& à l'huile. les ayant premierement fait tremper dans l'eau tranchées par roelles, afin d'en ofter la plus grande furce : elle fe feme tout du long du mois de Iuillet juilques à trois fois, afin que fi les vues ne rencontrent le temps propre, les autres puifent fuppleer; la Terre Sablonneufe bien amendéz, \& labonrée de trois Labours, eft ce qu'elle defre pour devenir bien grolle, il y en à gui parleur groffeur, cralent un Pain de deux fols; on les tirc de terre avant la Gelées, \& font conlervécs en lieu exempe dufroid comme los Nàvets

Pour recuellir la Graine, il ne faut qu'en laiterdes plus grolfes dans terme, elles y palferont l'Hyver \& graineront en la faifon: le plus cert in cit d'en replantar desplus grolfes à l'iffuèdes for:es Gclées.

Les Raves Noires ne funt pas beaucoup à prifer : elles fe veulent gouverner comme les petits Raiforts.

Lesperites Raves ou petirs Reforts, refement à tous les Decours des Luns, depuis que vons commenicures rolin pramiere Couche, jufques a tu mois d' $\mathrm{Cc}$ tobre: on les accommode diverfencut 
car fi vous les voulés auoir belles, claires, nettes, \& bien longues, il faudrs dans le temps que vous lemés vos Melons en quelque endroit de voftre Cou che alors quelle aura encor de la chaleur f) faire des trous de la hauteur de voftre doigt diftans de trois poulces, l'vn de l'autre; $*$ dans chaques trou y laiffer choir deux graines de katles, mettant vn peu de fablon par deffus, en lailfant le trou tout ounert, elles s'accroiffront de Ia hauteur de voltre doigt, par deffus ce quelles euffent fait, ne pouffans leurs preniores Fucilles, qu'apres qu'elles feront montées au Niueau de la Couche.

Quand vos Melons feront replantés, vous en pourrés femer fur la couche, \& auff en plaine Terre, le mettant par rayons.

Pour la Graine, vous laifferés monter des premieres femées, \& la cuillerés quand vous verrés les Goulfes d'en bas soururir, \& laiffer aller leur Graine: Vous la mettrez (comme les autres que i'ay dit) le long de vos Contr'Épaliers, pour la faire feicher \& s'acheuer de meurir: La meilleure graine que nous a yons vient de ces Hortillons d'amiens, qui en efeuent de trés bien conditionnéc dans leurs marais: ì la leuée il femble qu’elles ne 
I A O LE JA R DI I I E

foient pas bien franches: mais à la quatre ou fixiéme fuicille, elles fe fortifient merueilleufement, moyennant que l'on ne neglige de les arroufer.

Des Nauets, il y en a de plufieurs efpeces que je ne particulariferay point, je diray feulement que les petits font les meilleurs, \& les plus agreables au gouft, les autres cftant molaffes, \& ayans moins de faueir.

On les femes en deuxtemps, au renoutreau, ir au commencement d'Aouft: toute la difficulté qu'il y a, ceft de bien prendre fon temps; car s'il cft trop pluuieux, la graine créue, \& ne germe point; sil eft trop lec, elle ne leate point : C'eft pourquoy voyant qu'vue femaille aura manqué, l'on rebinera le gueret, \& on le refemera tout de nouseau: Quand is font leuez, mefmes quand ils ont deux, ou quatre fiieilles; fi le temps eft trop fcc, lesticques, \& puceons les mangent, \& $c^{\prime}$ eft à recommencer : C'eft pourquoy voyant qu'vne femaille aura manqué, faudra recommencer, comine je viens de dire.

Pour eftre bons, il ne faut pas qu'ils foient plus de fix femaines dans Terre, autrement ils deuiennent verreux fe deffeichent, \& font defagreables à màn- 
ger, eftans pleins de filets.

$L^{\prime}$ on les ferre pour 1 Hyuer dans la caue ou autre lieu exempt de la gelée, fans autre foin que de les mettre en monceau, ou par bottes.

Pour la graine, il faut choifir des plus beaux, plus lotigs, \& plus clairs, lefquels on remettra en Terre au renouueau, \& l'on arrachera la Plante quand on verra les premieres goulfes sounirir; la mettant feicher, \& l'on labrifera entre les mains fur vn drap, luy laiffant paffer le refte du jour au grand Soleil, pour en deffeicher toute l'humidité, puis l'on la nettoyera \& ferrera en lieu temperé.

Le Perfil fera mis auffi entre les raci. nes, quoy que fa fueille foit eftimable, vous feruant en plufieurs mets, \&e tenant place de Poiure, \&r d Efpice.

Les gelées eftant paffées, vous femerez le gros \& petit Perfil, le ponnaché, \& frisé, \& en Terre labourée profondement \& bien amendée, afin qu'il produife de longues \& groffes racines: Il fe feme par rayons fur des pianches, quatre rayons à chaque planche: puis la terre eftant rabbatuë dans les rayons, \& 10 gueret bien redreffé : on femera de l'Ognon pardellus, que l'un cnterreratiec 
I42 LE IAR D IN IE R

les denis du rafteau, en frappant doucament fur la planc e: $\&$ tout bien redrelféderechef, \& les fentiers netroyez, vous meterez lur chaque planche, enuiron la hauteur de deux doigts de petit fumier de vicille couche, tant pour amender la Terre, que pour empefcher que la pluye ou les arroufemens ne la batt:nt, \& ne la fallent creualler.

Or d'autant que la graine de Perfil. eft vn mois dans 'Terre lans leucr, l'Oignon aura loifr de s'accroiftre \& prendre affez de furce, pour eftre replanié: Quand vous l'arracherez pour le replanter, cela feruira de labour, \& de farclement au Perfil; \& par mofine mo en com. me il fera déja fort, vous pourrez l'éclaicir cù vouis verrez que !es plantes feront trop druës, afin qu'il en profite mieux.

Vous couperez de la fuieille quand vous en aurcz de befoin, fans que la plante en puine fouffrir aucun detriment.

Les racines fe laiffent dans Terre, \&r l'on n'en prend qu'au befoin, à caufe qu'clles groffiffent toújours, mefme durant l'Hyucr; vous en leuerez pourtant ce que vous croire $z$ en auroir affaire, de crainte que la Terre fe tronuant fcellée de lagele, vons n'en puificz anoir dars la se efficé. 
Pour la graine, vous en laiflerez monter quelque bout de planche, \& ne l'arracherez que tout ne foit meur, la laiflan feicher comme les autres.

Les Cheruis vionnent de graine, \& de plante, mais plus gros, \& meilleurs de plante, que de graine: l'on le fournit ordinairement de celles de Troyes ca Champagne comme les milleures.

Pour lesplanter, il fauten Terrebien bəfchée, st bienfumée, fairedes petits rayons, trois à chaque planche, de quatre doigis de profondeur, \&x concher dedans les racines de la Cheruis. à plus d'va demy pied l'vne de l'autre, pour leur dona plus de commodicé à sac. croire, adcuenir plasbelles.

L'on en tire de Terre à mefure que 1 on en a aff ire, laiflant le furplus qui groTtra toêjuurs, \& montera à graine en Ga faifen.

L:s Répunces, quoy que ce foit vne piance bien agreable au goult, \& qui s'apprefte pour eftre mangée en plufieurs façons, neantmois je ne m'arrefteray puint à dire la manicre de la gouvernur, d'autant cue l'on fo consente des famuges, fans le domer la peine d'en culituer.

I. I I mpinabours font der racines 
144 I E I A R I N I E R

rondes, qui viennent toutes par nœud's, $\&$ ruc l’on mange dans le $C_{2}$ reime en formc de fonds d'Ärtichaux.? ils ne veulent pas grande culture, \& pourueu qu'ils foient plantez en bonne Terre, ils profiteront abondamment.

On les feme de graine, \& on les planre aufli de racine: ils portent des fleurs comme de petits Soleils, dans lefquelles fe recueille quantité de graines, que les Medecins dilent en leur viage eftre prejudiciable à la fanté, c’êt pourquoy ils feront bannis des bonnestables.

Detcules les fortes d'Herbes Potegeres.

\section{$S$ ECTION. V.}

MOus commencerons par la BetteI blanche, ou Poirée comme par la plus grande de toutes les herbes porage$x$ is, \& de laquelle on tire plus abondamment que de pas vne autre.

La Bette-carde; ( ainfil la nommeronnous à l'imitation des Picards, qui meritent 'veritablement l'honneur d'eftre appellez les meilleurs, \& les plus curieux lardiniers pour les potages, que tous les autres de toutes les Prouinces de France; car foit que la Terre, ou le 


\section{FR A N ÇOIs.}

climat y apporte beaucoup, ou que ce foit par leur induftrie, \& trauail ; leurs herbages font tout d'vne autre grandeur \& largeur, qu'aux autres endroits : car puurla Carde de Berre j'en ay veu chez eux de huit poulces de large, ou peu moins, \& de longueur, \& époiffeur con. uenable à fa largeur ) fe feme au renouucau, les gelées eftant enticrement parfées : vous pourrez vous feruir des planches de vos Efpaliers pour ce fujer, puis quand elles auront fix füeilles : vous les replanterez en Terre qui aura efté profondement labourée dès l'Automne, \& meurie par les gelées durane l'Hyuer: Auant que de les replanter, on portera force fumier furla Terre \& la relaboure. ra-ton mettant le fumier au fonds de la jaulge, puis les ayant tirées de lcurs pepiniere, on leur roignera le piuot, \& on les replantera par planchics, deux rangs feulement à chaque planche \& à trois pieds l'vne de l'autre, faifant comvn petit rayon, ainli que j'ay montré cy-deuant, à la maniere de replanter toutes fortes de Choux, ce que je ne repeteray point icy, pour éuiter prolixité.

Si vous voulez qu'elles abondent en belles Cardes, vous ioignerez à les bicn 


\section{6}

biner, farcler $\&$ arroufer; quand veus teconnoiftrez qu' ell is en auront neceffité.

Pour les cueillir, vous ne les couperez pas, mais les arracherez de leur plar. te, en les tirant va peu de colté ; cela n'offenfera en aucune façon la fouche, au contraire elle groffira les reftantes, \& re parera fa ruine en furt peu de temps.

Vous ne replanterez pour Cardes celles que trouuerez yertes : car elles degenerent.

L'on en femera pendant tout l'Efté pour en auoir de tendres à mettre au potage, ou pour la farce.

Vous en femerez aufie à la fin d'Aoult, zufquelies vous laifferez palfer l'Hyucr en forme de pepiniere, \& al renouncali vous les replanterez pour auoir des Cardes toutes des premieres.

Il y a des Bettes Cardes qui font rouges mais fi vous en roulez auoir que ce foit plutoft par curiofité, que pour voftre vfinge, car elles nae font les Cardes que petites, \& eftant cuites perdent beaucoup de leur couleur rouge, deuenant pafles, ce qui fait qu'elles ne font fi agreables au manger, ny à la vecië, que les blanchcs.

Pour la graine, vous en laifierez mon. zer des plusblanches, \& plus larges, fans leur arracher aucune fueilles rous arre. 


\section{FR A N Ç-OIs.}

iterezle montant à quelque bon pieu, do crainte que fa trop grande charge ne le falle abatre, au prejudice de la graine, qui pourriroit au lieu de meurir.

Deux plantes au plus fifiront pour vousen fournir amplement : vous l'arra- cherez par vn beau temps, (quand vous jugerez qu'elle fera meure far la couleur jaune qu’elle prendra, ) \& la laille. rez bien fecher, puis la froillerez auec les mains fur quelque nappe, la nettoyes rez de toute ordure, \& la ferez encor bien feicher, de crainte qu'ellene moififfe, car comme ille eft fponyteufe, aufi bien que celle desBettes-rancs, cllegardera long temps fon humidice.

11 y a vie petite efpece de Battes que l'on nomme Arroche, ou Bonne-Dame, qui eft fort agreable au manger, rendant le potage trés-beau, \& qui porte lon beu. ve d'elle-mefme.

Elle veut pareille culture que la Berte. Carde, referué qu'elle fe p'sute plus prés: elle vient bien aufli fans eitre replantée, fuftit qu elle foit farclée, si bi. née dans le befoin.

Il y a plulieurs efpeces de Chicnró:s fraiches, qui font differentes en fieillages, \& en grandeur, mais poumant femblables au gout, it guil gou.

$$
G \text { if }
$$


148 LE IARDIN I ER uernent tout d'vne mefme façon.

Elles fe femeront au renouucau, fur les planches des Efpaliers: \& quand elles auront fix fiieilles, on les replantera en Terre bien amendée, à la diftance de dix. huit poulces l'yne de l'aurre, en lèur rognant le piuot: lors que par leur accroiffement elles courriront toute la Terre, il les faudra lier à la manicre que $j$ 'ay dit cy-deuant : quand j'ay traité des Chicons; qui eft de les lier non pas toutes à main, c'eft à dire fortes \& foibles, mais prendre les plus auancées les premieres, \& laiffer fortifier les autres; je vous y renvoye, afun d'éuiter les redites; c'eft en la Section treiliéme de ce fecond Traité, en l'article des Laictuës, où vous verrez auffi la maniere de les blanchic promptement auec des pots de rerre.

La Chicorée fe blanchit cncor d'vne autre forte: durant les grandes Chaleurs, quand vous verrez quau lieu de fe pommer, elle voudra monter à grai ne, vous creuferez la Terreà colté de la plante, \& fans l'arracher vous la colicherez dans terre, ne laiffant fortir que le bout des fucilles, elle fe blanchira en fort peu de temps, \& cela l'empefchera de mónter à graine.

Pour plus grande propricté, il eft bow 


$$
\text { FRA Mç OIS. I } 49
$$

de les lier auparauant, afin que la Terre ne tombe pas entre les fuieilles: ce qui donneroit tant plus de peine à les bien lauer auant que de s'en fernir.

Vous obferuerez de les coucher toutes d'vn cofté, les vnes fur les autres, comme elles ont efté plantées; commençant par celles du bout de la planche, \& en continuant, couchant les fecondes fur les premieres, \& les troifiefmes fur les rangs iufques au bout.

Ie tronue encor deux autres manieres de les bien blanchir pour l'Hyuer. La promiere, eft qu’a l'entrée des gelées vous les licrez à l'ordinaire, pour au bout de huit ou dix jours, les ayant arrachées, vous les enterrerez dans la couche, qui vous aura feruy a éleuer vos femences, faifant vil petit rayon de trauers la conche, de la hauteur de voftre plante, qui fera de huit poulces ou enuiron; commençant par vn bout ; vous coucherez dedans vos plantes cofte à cofte, fe touchant l'vne l'autre fans fe preffer pour tant, \& vn peu en penchant; cela fait, vous les conurirez du petit fumier de la mefme cottche, en faifant vn autre rayon pour vre leconde rangée, dans lequel vous coucherez des plantes, comme au premier, \& ainfi en continuant, autant 
Ijo LE IA RT I I I E Rे

que vous aurez de plantes: par aprés vous counrirez le tout enuiron la hauteur de quatre doigts, de bon fumier chaud tout recentement tiré de l'Efcurie \& en peu de temps elles blanchiront : fi vous voulés les coururir de quelque paillatfons en forme de toict, pour les preferuer des grandes pluyes, cllcs dureront fort longcemps fans fe potrrir: Quand vous en voudrez prendre pour voltre viage, vous commencerez par les dernicrs qu'aurez enterrées, \& prenant tout à main fans diftinction, vouslestirerez de rang, \& fur le lieu mefme les éplucherez du pour ry, ou de e: quilera roircy par le fumier, auant que de les mettre dans la corbeille, pour les porter à la cuifine.

La feconde maniere de les confertuer plus long - remps, fera de les enterrer commedelfus par rayons, dans le fablois à la caue : leur mettant la racine en haut, de crainte que le fablon ne grille entre les fueilles, \& qu'il ne s'en trouue dans les plats, quand on vous la feruma: Il n'eft pas necelfaire a'y mettre dufumier par deffus, pourueu que le fablon couure la plante de quatre doigts de haut, it fuffira : \& quand vous les tirerez pour voftre vfage, auant que de les dreffer, zous les fecouërez bien, la racine enhaut, 
poir faire tomber tout le fablon, qui pourroit eftre aux fueilles; vous les prendrezauffi toutes à main, comme elles fe prefenteront dans leur rangées.

Il y a de la Chicorée quir fe ferme d'elle. mefme fans eftre lièe, qui eft d'vne fort petite efpece, mais beaucoup eftimable pour fa bonté.

Quant à la graine, yous laifferezmonter des plus belles plantes, \& particulierement de celles que vous verrez quả veulent blanchir d'elles-mefmes, \& fe pommer fans eftre liées: vous la laiffercz bien meurir, mefmement paffer de meurir, car elle ne tombe pas ainfi que beaucoup d'autres : at contraire, quand apres l'auoir laiffée bien fecher, vous la porterez fur l'aire de la grange, encore aurez vous allez de peine à la tirer dedans les nœuds à grands coups de fleau.

L'endiue ou Chicorée fautuage, fe gouuerne comme la franche, \& auec moins. de peine, la femant feulement dans vn petit rayon, \& la farclant, binant, \& éclairciffant en faifon.

Pour la blanchir, on la coure fimo plement de fumier moyennement chaud, elle fe tire de Terre au commencement des gelées, \& fe ferre dans le fablon a la caue comme les autres racines; mais

G iiij 
352 IE IARDINIER

auant que de l'y mettre, il faut qu'elle foit prefque blanche; fa racine eft beaucoup eftimée, \& j'ay douté fi je la deuois mettre au rang des racines, mais j’ay jugé plus à propos de la placer auec la franche, à caufe de la conformité qu'elles ont, tant a l'accroiffement, qu'à la façon de grainer.

De l'Ozeille, nous en auons de plufieurs efpeces, quifont, la grande, ou $l^{\prime}$ Ozeille à la mequenne, ou à la pareffe; d'autant qu'vne feule fueille peut fuffire pour vn potage, attendu qu' elle eft $d^{s} v$ ne fi prodigieufe grandetir, qu'elle 2 des fueilles qui ont jufques à fept poulces de large, \& quinze ou dis huit poul. ce de long, c'eft vne efpece que l'on a apporté des $\mathrm{Pa}$ ais Bas, j'en ay eu des premiers.

La feconde efpece, eft vne autre grande Ozeille femblable à la patience.

La troifiefme, eft celle qui ne porte point de graine, mais de qui la plante s'élargit dans terre, en produifant de petits jettons à cofté defquels on fe fert pour plant.

La quatriefme, eft la petite Ozeille que nous auons dés fil long-temps en vage.

$\mathrm{L}_{\text {a }}$ cinquiefme, eft l'Ozeille ronde, 
grande \& petite, qui ne graine point auffi, mais on en tire du plant de certaines trainalfes qu'elle fait, qui couurent toute la Terre : \& par des rejettons qui font autour de la plante, que l'on partit en petites touffes, pour en faire des planches.

La fixiefme eft la Surelle, ou faulage qui fe troulue dans les haut prez, que Ion prendra la peine de cultiuer dans les Iardins.

Il y en a vne feptiefme qui a la fueille en trefle, que l'on nomme Alleluya: elle eft fort delicate \& agreable à caufe de fon acidicé, toute femblable à $1^{\prime} \mathrm{O}$ zeille, quant au gouft; fort excellente dans les potages, farces, \& falades, d'autant qu'elle a toutes les mefme qualitez \& gouft que les autres Ozeilles.

Elles fe pcuuent toutes femer à l'iffuë des gelées, par petits rayons, quatre à chaque planche : vous foignerés qu'elle foit bien farclée de toute les herbes qui l'eftouffent : quand elle fera vn peu forte, vous l'éclaircirez afin qu'elle en profite micux : \& fir vous voulez, de ce qu'arracherez vous en ferez d'autrcs planches: mais le mcilleureft, fivousen defirerreplanter, de prendre du plus fort plant, \& $2 u$ commencement de l'A utomne, o:s 


\section{If 4 LE IAR DINIER}

Printemps; en faire des planches à part: elle vient bien d'vne façon \& d'autre, dure fort long temps en ${ }_{a}$ bonté, mefmes jufques à dix, ou douze ans, apres lefquels il fera bon d'en replanter ailleurs, d'autant que la terre s'ennuye d'eftre toûjours charrgée d'vine mefme plante, \&t fe plaift en la diuerfité : joint auffe que les racines, sentrepreffans les vns lcs autres, ne tronuent pas de fublance fuffifante pour leur entreticn.

Elles fe veulent labourer au moins trois fois l'An: \& à la derniere qui fera au como mencement des fortes gelées, vous repandrez par deffus du petit fumier: Les crottes du poulailler luy font auffi trésbonnes, \& la font profiter à merueille.

A ce dernier labour; l'on arrache toutes celles qui par quelques graines tombées font hors de lcur rang, \& auffi on chattre la ronde, \& coupe-t'on à toutcs fizeilles ć tiges tout prés de terre, auant que de les couurir de ces fumiers.

Pour la graine, elle ett facile à recuieillir en celles qui en portent, car le plein Elté venu elles montent, \& quand vous verrez qu'elle fera meure, vous couperez les tiges prés de terre, puis eftant bien feichéc, elle quitterafacilement fes bourfes, vous la netroyerez bien, \& la crrercz. 


\section{FRA çOIS.}

La Patience fera gouuernée comme l'Ozeille, c'eft vie plante qui n'eft fi delicate all manger, neant-moins vous ne lailferez d'en auoir quelque planche, afin que voltre Iardin ne manque d'auctrne chofe.

$\mathrm{L}_{\mathrm{a}}$ Bourrache, à caufe de fa vertu troǘ uera auffi lieu dans voftre Iardin, quoy qu'elle gafte la couleur des potages, en les noirciffant : les fleurs font fort agreables à feruir fur table, \& fe peuuent mettre fur la viande, fur les potages, falades ¿2 autres aprets : d'autant qüù caule de feur grande douceur, les mange qui veut, fans qu'elles donnent aucun degoult.

Elles fe fementau renouneau, comme tes autres herbes, \& re peuuent laiffer dans terre, leurs racines fupportans les gelées, \& repoulfans au Printemps: Les Marailchers de Paris arrachent tonie la plante, \&z en fement plufietrs fois durant l'Année, à caufe qu'elle en eft plus tendre.

Pour fon gonturnement, it fuffira de la biner legerement, \& la bien farcler.

Pour la Graine vous laifferez monter des plus belles plantes, \& eftant bien meurie fur le pied; vous la cueillirez, \& ferrerez ainfi que les autres.

La Buglofe, fera gouuernée tout de la 


\section{If 6 IE IARDINIER}

mefme façon que la Bouroche, c'eft pourquoy jene m'y arrefteray point.

Le Cerfueil, outre celu que je vous ay dit cy-deuant qu'il falloit femer fur les Couches, pour compofer lespetites Salades, à la fortie de l'Hyuer il fera bon de mois en mois, d'en referuer de nouneau (quoy que peu) à caufe qu' il en fera plus delicat que quand il eft vieil femé : Les Bandes de vos Efpaliers, \& Contr'Efpaliers pourront feruir à cét effet, dautant qu' il ne peut nuire à vos Arbres par fa petiteffe, \& pour le peu de fubltance qu'il luy faut à fon accroilfement, \& encor pour le peu de temps qu'il fejourne en un lieu. Vousen en laifferez monter à Graine quelque bout de planche, qui fuffira pour vous en fournir am plement, la laifferezbien meurir fur le pied, puis vous l'arracherez, où couperez, \& la feicherez parfaitement, a vant que de la ferrer.

Il y a une autre efpece de Cerfueil d'ESpagne, qui fe nomme Mirrhis-Odorate: fa feuille reffemble toute à la Ciguë, eft fort agreable au gouft, ayant fon parfum femblable à l'Anis-Vert, \& encore plus agreable en le mafchant petr.

Au Renouveau, quand de $\mathrm{Ca}$ vieille $\mathrm{Ti}$ ge il pouffe fon jet, on le couvrira de fumier menu, puis de chaud par deffus 


\section{FRA N C OIs.}

pour l'eftouffer, afin d'eftre mangé en Salades: il elt de beaucoup plus agreable que le Perfil de Macedoine, ny que le Sceleri d'Italie.

On le femera au Renouveau en quelque endroit à part, \& on ne le labourera en alicune façon tant qu'il foir levé, mais on le faclera feulement à mefure que therbe y pouffera, dautant qu'il eft par fois une Année fans fans fortir de Terre.

Pour la Graine vous la recuillirez en fa faifon, \& la gouvernerez cóme les autres.

Le Perfil de Nacedoine fera mcinagé tout de la mefme façon que je vicus d enfeigner au Cerfueil d'Elpagne, excepté que fa graine n'eft pas fi long-tempsà lever \& qu'il ne fe mange qu'étoliffé fous le Fumier ou fous des pots de Grets, comme la Chicorće.

Le Celerid Italie fera traitté de la méme maniere, fon montant eft ce qui eft de plus exccilenten fa plante, à caufe de fa delicatelle \& tendreur.

Ces trois dernieres Plantes ne fe velllent femer tous les Ans, maisfe conferver dans terre fans crainte que les $\mathrm{Ge}-$ léesles falfent perir.

Du Pourpier, i'en trouve de quatre fortes: Le vert, le Blanc, le Doré, a'agueres apporté des Inles de S. Chriftophe, 
I5\& LE IARDINIER

qui eft le plus delicat, \& la 4 . êt la Pourceline, ou petit Pour: pierfauvage, le moins à prifer, ta terre le produifant naturcllemened'elle-mefme, fansaucuntravail.

Il fe feme au Renouneau fur la Couche, \& tout le long de l'Efté, pour en aubit: toufiours de tendre.

Fourle femer, il faudralabourer laterre, puis la bien drelier par tout, \& efpandre deffus voftre Graine la phis claire que vous pourez, d'autant cu'à caufe de fa petiteffel'on y met toûjours plus qu'il n'en faut, \& eftant femée, vous ne l'enterrerez d'autre façon, finon en battant fur la plan* che auce le dos d'vne paefle de bois, ou d'vn battoir à lexiue: Onl'arouferale plus proprement que l'on pourra pour ne faire des trous à laterre, il leve en fort peu de temps, c'elt pourquoy l'on ne le lailftra manquer d'eau dans fon commencement.

Il fe replante pour en tirer de la Graine bien conditionée, \& pour avoir de plus. gros cottons à confire au Sel, defquels on fe ferten Hyver dans les Salades, \& dans les Potages.

Pour la Graine, vous jugerés que elle fera meure quand vous la verrés bien noire, alors vous arracherés la plante \& $l_{2}$ mettrés fur quelque drap fe fannir, \& feicher au Soleil: le foir vonu, vous ls 


\section{F n A}

frirerés à couvert dans le mefme drap, la remettant le lendenain au Eoleil; \& tous les jours en cortinuant de mefme, ccla acheuera de meurir colle qui ne l'estoite pas : puis apres vous la broycrés dans les mains, az la mettres fir quelque autre - linge à part pour bien feicher, auant que de la ferrer : vous remettrés dcrechef feicher les plantes quelques jours durant, car elles vous rendront encore beaucoup de graine qu'elles n'auroient pas quittée.

Vous remarquerez que la graine nou"uelle n'eft pas li bonne à femer que celle de deux, trois ouquatre ans.

Les Epinars fe tromuent de trois efpeces: des grands qui no ont pas la fueille fa poinctuë que les petit, \& desblonds, qui font la troifiéme efpece: ils fe veulent femer au commencement de l'Automne, afin d'ellec fortifiez a uant l'Hiyucr; fi vous voyez qu'ils pouthent par trop, rousen pourez coliper pour les potages, \& pour la patilferie, ils leront de beaucoup plus tendres que dans le Carefme oil l'on en mange ordinairement: La maniere de les femer eft par planches en petit rayon, guare à chaque planche: On les farclera proprementquand ils feront leuez, \& ofteraon ceux qui par hazard de quelque graine tembée, feront leuer entre deux rayons. 
260 IF IARDINIER

Vous en referuerez quelque bout de planche pourlagraine, coupant aux autres endroits ce qu'en aurez de befoin, \& dant le Carefme, vous arracherez la plante entiere pour tont employer à la cuifine, n'y rognant que la racine.

La graine fe troulue de deux fortes, de picquante, \& d'autre fans picquans, toute ronde : celle.cy fait les Elpinards blonds, \& plus delicats que les autres.

Des Felues, Poix, cautreslegumes,

\section{SECTION VI.}

TL y a trois fortes de groffes Fenes, cel1 les que l'on nomme à Paris Feues de Marais, lefquelles viennent larges, plat res, \& de couleur fort blonde: D'autres plus petites beaucoup, femblables aux premieres, fauf qu'elles font plus rondes: Et d'autres encor plus petites \& toutes differentes des premieres, d'autant qu'clles font prefque rondes; \& de couleur grife, ou rougeantre : ce font de ces Feures que l'on donne aux Cizeuaux, \& que l'on fait mouldre pour employer en plufieurs befoins.

Iene veux traitter icy que de la maniere de gouuerner les plus grofles, laiffant les 
petites comme de peu de valeur, \& je diray, que les opinions font bien diuerfes pour le temps, \& la façon de les femer.

Aucuns les fement dés les Aduents de Noël, \& tiennent qu'ils en ont des premieres bonnes à manger: Autres attendent jufques à la Chandeleur : Et les autres veulent que les gelées foient entierement palfées, auant que de fe mettre à ce labour. Chacuna fes raifons particulieres, \& difent que c'eft à caufe des puceons qui en mangent la cime quand elles font en fleur : pour moy qui ay toû。 jours en vifée le plus certain, j'atends que l'es gelées foient paffées, \& je fonde ma raifon fur ce que la faifon amene tout. Ce n'eft pas que je vous veüille diffuader d'en femeraux Aduents, \& au mois de Feurier; mais je vous confeille d'en faire peu, referuant la plus grande quantité quand les gelées feront palfées: d'autant que comme il eft necelfaire de les mettre en la meilleure Terre, \& la plus balfe que vous ayez: elle fera mal en labour aux deux premiers temps, à caufe qu'elle retient \& garde plus fon eau, que les terres legeres.

Auant que de les femer vous choifirez celles qui font les mieux conditionnées, $\&$ lesplus faines: vous les mettrez trem- 
IG 2 LEIADINIER

per vn jour ou deux, dans le jus du fü: nier, elles s'enfleront extremement, \&: s'auanceront de germer plus qu'clles ne feroient en dix, ou donze jours: Outre que, ne fejournans paslong temps dans Terre fansleuer, la vermine oura moins de temps de les endommager, \& d'abondant, ayant trempédans ce jus, elles s'im: bibent de la bonne qualité du fumier, qui les fait vegeter plus abondamment.

Pour les femer, il faut que la Terre ait efté labourée allant l'Hyuer, nettoyée de toute herbe, puis auec la fourchel'on fera vne jaulge, fur l'ados de laquelle ( \& non pas au fonds) vous placerez vos Feues, à demy-pied, ou vn peu plus l'vne de l'autre: puis aprés vous ferez vne feconde jaulge; $8 x$ de la Terrequ'en leuerez vous cosurirez vos Feues: puis vne troifiéme, \& furcette troifiéme vous. placerez des Feues comme à la premiere: ainfi en continuant de deux en deuxjaulges, vous y mettrez des Feues: il faut eftre curicux de mener la jaulge la plus. droite que l'on pourra: ofin que l'on puilie les biner, farcter, strogner : fans rompre les tiges en palfant dedans.

Il y en a d'autres, qui aprés auoir biem labouré, \&-dreflé leur Terre la diuifent par planches, \& les fement au plantoir: 


$$
\text { FRA } \bar{N} C \text { O I s. }
$$

anais l'autre maniere me femble moilleure, d'autant qu'elle rend la Terre plus efmiée, ou mauble, ou pele, com. me vous la voudrez nommer, maccommodant au langage de plufieurs Pays, pour la facilité de me faire entendre.

Dans leur accroiffement, guand rous verrez que l'Herbe les roudra fuffoquer, vous les binerez, \& farclerez proprement, fans les endommager; \& desja grandes, vous remarquerez que les puceons, ou moucherons, paroiftront au coupeau de leur tige, sattachans à las partie la plustendre: alors vous les rognerez, cmportant les puceons, auce le plus tendre du jet, où ils font attachez.

Vous mettrez ces roignures dans quelque boiffeau, pour eftre jettées au feu: ou bien vous les enterrerez dans la forfe au fumier, ou en quelque autre licu fort éloigné de vos Fcues, car ils y re. tourneroient.

Vous deft inerez quel ques planches particulieres pour en manger en vert, fans cueillir des gouffes dans toutes les planches; \& quand vous aurez entierement dépouiillé quelque plante, vous la couperez prés de Terre, afin qu'elle jette de nouneaux jettons, qui porteront leur fruit dans l'arriere failon. 
164 LE IAR DINIER

Pour la Semence, vous les laiffercz bien fecher fur pied, jufques à ce que les goulfes \& la tige foient toutes noires; \& les arracherez à la grande chaleur du jour, les ferez battre au fleau à petits coups, \& les ferez vanner à voftre commodité.

Le foarre, ou chaulme, que vous en tirerez, ne fera brûlé, quoy que la cendre en foit très-bonne, mais fera mis pourrir auec les fumiers pour les ameliorer : mefmement fi vous voulez bien amander de la Terre, femez y des Feues; $\&$ quand elles commenceront à defleurir, labourez le tout enfemble terre, \& Feues, fansfonger à la perte qu'il y peut auoir; car cette maniere de fumier eft vn merueilleux amendement.

Il fe trouue des groffes Feues qui font $d^{2}$ vn rouge brun, mais elles ne font fi delicates que les blondes.

Les petites Feues d'haricot, ou callicot, ou bien Feues rottes font de deux efpeces, de blanches, \& de colorées, parmy lefquelles il y en a aufi de blanches, mais plus petites, \& rondes que ne font pas les grandes blanches.

Pour commencer par les grandes vous les femerez en quelques planches à part, quatre rangées à chaque planche, afin 
d'auoir plus de commodicé de les ramer, que fi elles eftoient femées en confulion: Vous en deftinerez quelques-vnes pour en manger en vert, en lailfant les autres pour les mancher feiches, \& pour la Semence: Quand vous les cueillirez, vous prendrez garde de n'offenfer ny corrompre la tige, afin qu'elle vous en produife jufques à ce qu' elles feichent fur le pied.

Les colorées qui font plus petites, fe fement ordinairement en plain gueret frais labouré : que l'on herfera fans y apporter autre foin qu'aus grains qui font en plain champ: finon que huit, ou dix jours apres qu'elles feront leuées, il eft bon de les biner, \& n'y plus toucher, jufques à ce qu'elles jettent des trainaftes, qui eft au commencement de Iuillet (lefquelles il faudra rogner, ou chafrer, pour faire mieux profiter les goufes qui font au bas de la tige; \& pour éuiter que en lians les vnes aux autres, par trop de ramage, elles ne verfalfent, \& ne pourriffent celles de deflus, au lieu de meurir.

Ces fortes de Feues, ne defirent la Terre ii forte que les Feues de Marais, mais plutoft la Sablonneufe.

Elles vculent eftre femées an commencement du moisde May, \& arrachées à 
166. LE IA R D I I I R

mefurc que les plantes feichent, les fai. fantbattreaufleau, comme j'ay dit cydeuant aux Feues de marais : carli vous les cueillez plus vertes, vous aurez grande peine à troutuer les lieux propres pour les metre feicher, qui feroit vn granden. baras, fi vous en auiez beaucoup.

Quant aux branches qui lont ramées, d'autant qu'elles montent au haut de leurs rames, \& font long-temps à tohijours porter, il fera bon de cueillir les goultes que vous verrez eftre feiches, à caule guiclles ne meuriflent pas toures en melme temps, \& qu'il en peut arriuer decux inconueniens: Le premier, queftant pallées de meurir, la goulfe souuriroit à la grande chaleur du jour。 \& Jairteroic tomber les Feues qui font dedans: \& le fecond, que s'il venoit de grande pluyes, le parchemin de la goulfe eltant mouillé, il sattacheroit aux Feues, parvne certaine glus qui s'y fait, \& nes'en détacheroit plus, endommageant les Feues par vn moyli quiles tacheroit, \& les rendroit defagreables à la veuë, \& cncor plus au manger: outre que l'on feroit obligè de les écoller à la main, ce qui feroit perdre beaucoup de temps.

Volis tircrez toutes les noires, \& touus cuins qui feront mafles de noir, \& 
Slanc; dautant qu'elles deuiennent:nerc quen cuifant elles teignent lo boiill nt.

Vous ferez étar des rouges par dellus toutes les autres, à caule de leurdelicatelle? furpalfant de beaucoup les blanches, quoy qu'à Paris elles foient le plus en eltime.

Les Pois fe trousent de plufieurs efpeces tres differents: fçauoir les Pois chauds ou hatifs, les naims, les gros blonds, ceux à cul noir, les verts gros \& perits, ceux à couronne, ceux lans parchemin de deux fortes, ies ciches auec er lans parchemin, \& les Pois de cous les mois, les gris, se les taulpins \& lippins.

Je troulue fort à propos de particularifer lewr gouluernement, quoy que ce loir vane plante peu dificile à elieuer, lasantmoins il ne lera hors de propos pour vat plus grande inferuction.

Ily a iruis manieres de femer des Puis par planches, en faifant quatre ou cinq rayous à chacune, felon l'efpecedes Pois que vous y voulez femer par torches ou bouguets, \& en confulion.

Les Pois chauds, ou haftifs, re veulent femer dés la Chandeleur, ou bien pea apres los grandes grlées.

1.2 Tcrre biblonnewía elt selle quils 
I68 LE IARDINIER.

fouhaittent pour hafter leur accroifement, \& s'ils font fur quelque colteatz expofé au Soleil du midy, cela les auancera encor de beaucoup. La cofte de Charenton, \& faint Maur prés Paris, en nous en donnant de fort bonne heure, nous en fait paroiftre l'experience, \& tout le fecret ne gift qu'à les rebiner plufieurs fois, cela les auance merueilleufement.

Si vous les femez par rayons, ce vous fera vne grande commodité pour les biner, troumant place entre deux rayons pour loger vos pieds, fans endommager les jets: \& eftans déja grands, vous les pourrez ranger les vns aprés les autres, pour auoir plus grande commodité à les rebiner plufieurs fois, \& pour la facilité de pounoir en cueillir les gouffes ou colfes en leur faifon, fans endommager la plante.

Si vous les voulez femer par trochets, vousferez destrous auec le plantoir, vn bon pied prés l'vn de l'autre : \& dans chacun vous y mettrez fix, ou huit Pois, ils fortiront hors de Terre, \& \& s'accroiftront aunant que de s'épandre fur le gueret, vous donnant vn loifir fuffifant pour les biner pluficurs fuis fi vous voulcz. 
Quant à ceux que l'on feme ch confufron lur le gueret frais labouré, ou ceux que l'on feme fous la raye à charruë: ils ne vous donnent pas tant de loilir, a caufe qu'ils s'eftalent de tous coltez, ar 1 on nel:s peur biner qu'vne fois, fans fo meitre en d'anger d'en gafter beaucoup auec les pieds.

Tous Pois de la grande efpece (comme blancs, verts, à couronne ceux fans parchemin \& chiches) fe veulent femer par planclies, en petit rayon, quatre rangécs à chaque planche, pour la commodité d'y mettre doux rangs de rames, lefqualles Ceruiront pour appuyer deux rangées de Poix, \& tant plus vos Pois feront de grande ef́pece, tant plus vous tiendrez vos rames fortes, \& hautes: d'autant qu'ils monteront julques au bout, yctant des coffes à chaque næud, parciculierement la grandecfpece : ceux fans parchemin, dont la culle deuient croche, leqquels chargent extremement; \& jettant des rameatix à chaque nceud depuis le pied, qui portent fouticnt a.1cant de colles que le maitre brin des au. tres : C'eft vne efpecs quic vous deuez beaucoup eftimer puur ia delicateffe, \& qui fe peut nianger vert, E auicap petit anif ju. Liskanes. On les appille 
:70 LE I A R D INIER

Pois de Hollande, \& eftoient fort rares il ri'y a pas long-temps.

Si vous voulez auoir de tres gros Pois il les faudra femer en bonne terre, \& leschaftrer quand ils feront à la hauteur de quatre pieds : mais aufi le mal eft, qu'entans femez en terre forte, ils ne cuifent pas fi bien que ceux qui font produits dans le fablon, qui eft la vraye terre qu'ils defrent pour eftre bien conditionnez.

Vaus ne metrez vos plancines de Pois ramez toutes les vnes attenans les $2 u-$ tres : ains, lailferez vne planche entre deux, pour domner de l'air à vos pois, autrement ils seftoufferoient, pourriffans par le bas: \& dans ees planches d'entre-deux, vous y femerez de quelques fortes de racines cy-deuani deforites, lefquelles profiteront bealicoup, à caufe de la fraifcheur, \& onbre qu'elles receuront, par la hauteur des Pois.

Vous deftinerez aufi quelques planches particulieres pour en manger en vert : \& ferez cueillir les coffes par perfonnes non eftourdies qui auec patience les arrachcront, ou couperont proprement de leurs tiges, fans l'endom. mager : afin d'cn tirer tout ce que la Hhate en pourra fournir, \& ils vous du, tsiont long-temps. 
Quant aux Pois de la petite efpece ( comme blancs, verts, gris, haftifs, naims, \& à cul noir ) vous les pourrez femar en plein champ à la charruë; d'autant que ne failans pas grand ramage: ils nio s'eltoufferont pas.

On les feme en deux façons, en gueret frais labouré, qui aura eu vne premiere façon auant l'Hyuer, ou bien fous rayes: c'eft à dire quauant que de labourer la Terre, l'on feme les Pois fur le champ; puis en failant les rayes, la femence tombe au fonds de chaque raye, ¿z auec l'ayde duSoc, eft recoutuerte par la Terre qui fe retourne deffís.

Cette maniere de labourer fe pratique pour deux fins: l'vie pour les loger fraifchement quand la Terre eft par trop lege. re: \& l'aucre peur empefcher les Pigeons de les manger : car à ceux qui ne font que herfez fur le gueret frais, ils y gratent commedes poulles, \& mangent la plafpart de la Semence.

Il y a encor vne autre methode de femerdes Pois, que les Picards pratiquent: ils ont vne forme de hou ë platte, à la maniere des Vignerons d'aupres Paris, dont les Vignes font dans les Terres glaiies, ou dans les fablons: Cét outil eq tout femblables a leurs hoüs, quand ils 


\section{$I 7$ LE IARDINIER}

les quittent, lors qu'elles font trop vfées par les coltés, faifans vne poincte dans le milieu : \& pour vous en donner vne intelligerce plusfacile, il refemble proprement à vn foc de charuë à tourneoreille, \& s'en ieruent de melme façon que l'on laboure les guerets à tourneoreille : c'eft à dire, qu'ils ne font aucunsfillons, ou fentiers, non plus qu'aux guerets, où il n'y a que celuy qui diuife le voifin, d'auec le voifin.

En terrelabourée de puis peu, déchar. gée de toures mauuaifes herbes, \& bien redreffée; ils font vne raye auec ce huet: (ainf le nomment-ils ) en reculant, $\$$ attirant la terre, elle fe fepare des deux coltez: dans ce rayon, ils y fement des Pois à difance raifonnable: puis cn recommençant vin fecond rayon, le huet recouure de torre celuy qui crt enfemencé, puisle troifiéme, le lecond, \& aini continuant jufques au bout de leur piece : cetre maniere eft expeditiue, \&z com. mode pour les pounoir biner auec facilité fans marcher deflus, lors qu'ils font: déja forts: il fe feruent auffi de cette maniere de femer aux Feues dotoutes efpeces, Raues Ozeille, Poirée, \& beatcoup d'autres herbages profondant dai.s icre plus aux vnes qu" au: autres, fe- 


$$
\text { FRA } \mathrm{N} \text { ç Is. }
$$

lon la force de la Semence.

Les Pois de tous les mois, ( ainfi dits, parce qu'ils durent prefque toute l'Année, fleurifans continuellement) yous les femerez à l'abry du mauuais vent en quelque endroit de voftre Iardin, pour on auoir de bonne heure.

Pour leur gouuernement, il n'y a autre curiofié qu' aux autres, finon de couper proprement les coffes eftans en vert, n'y en laiflans feicher aucune, \&à me. fure que rous verrez qua il y atra quelque jetton, duquel vous ne pourrez plusefperer de colfes, le couper.

Il faudra auoir grand foin de les arroufer, particulierement durant le mois d Aouft, \& leur ferez yn abry auec des paillaffons durant les grandes chaleurs, pour les garantir de la force des rayons du Soleil.

Les lupins ou Pois taulpins, ( ainfi dits à caufe que la taulpe fuit le lieu où ils font femez) ce font Pois plats, \&z ronds comme vne balle de Piftolet applatie, dans les Galeres, on les appelle Pois d'ECclaues, d'autant que l'on en nourrit les Forçats; ils font amers au gouft, \& veulent tremper long-temps auant que l'on les falfe cuire: ils viennent par gouffes, attachées à la tige comme des Feues, 
874 LE IA R D I IER

\& chargent beaucoup : En Efpagne ils en fement des champs entiers, \& en nourriffent le beltial.

Ils veulent eftre femez en rayon, à quatre doigts l'vn de l'atre, \& quatr rayons à chaque planche, veulent aumi la terre mediocre.

Les Lentilliers fe fement au mefme temps que les Pois, en gueret frais labouré : fi vous kuy aluez donné rn prea mier labour auant l'Hyuer, cllesen viendront beaucoup plus belles: la Terre fablonneufe leur eft agreable, l'on les cucillira cftans meures, \& les laiferaton jaueler: Vous les pourrez laiffer en la grange tant que vous voudrez fans les battre, d'autant que les fauris ne les mangent point, ny elles ne forr pas fu.. jettes aux verts comme les Pois, quien font continuellement rongez, tant qu'ils. font dans la colfe : c'eft pourquoy il faudra vfer de diligence à les faire battre promptement : Aucuns au fortir du champ, par vn beau jour, les battent dans la ruë fur vne belle place au grand. Soleil, qui aide beaucoup à les faire ef. colfer; car de les engranger, c'eft beaucoup de peine:outre qu'ils fe refuënt comme toutes fortes d'autres grains, \& ramoliffent leurs colfes, ce qui fait qu'ils 


\section{F. A A ç O I 5 .}

ne fe battent pas if bien: Vous pourrez pourtanten engranger des gris, pour les donner aux Cheuaur en gerbe, cela les desagache, \& reftaure s'ils font décheus so extenuez.

Des Oignons, Airs, Ciboules, Poirreaux, Heroes Odoriferantes, o autres commoditezdu I ardin, qui ne Sont comprifes aur Cbata pitres precedens.

\section{SECTION VII.}

7 "Oigion fe troune de trois couleurs. L le blanc, le palle, \& te pourpré ronge : je dis de trois couleurs, car je n'eltime pas que ee foient trois efpeces differentes, veu qu'ils font prefque fonblables en gouft, lailfant à juger de leurs qualitez aux Simpliftes.

Outrece que je vous ay fait cy-deuant femer des Oignons auec le Perfil, vous en femerez encore d'autres fur quelque planche à part : Et quand il fera gros comme le tuyau des plumes de Poules, vous les pourrez replanter en rayon au plantoir, afin d'en auoir de trés gros.

Si vous en laiflez fur la planche oùl'aurez femé, il deuiendra plus petit, \& fe. 
376 LE IAEDINTET

monftera pluton hors deterre en failon, que celuy qui aura efiè replanté.

Durant les grandes chaleurs d'Efté, it voudra monter à graine ce qu' il fait preuenir en pilant aux pieds le montant, cela l'arreftera, \& fera groffir l'Jignon.

Quand vous verrez qu'il fera hors de terre, qu'il aura la fueille bien feiche, \& qu'il fera comme l'on dit, bicn Aoulté, alors vous l'enleuerez entierement, recherchant iufques aux plus petits dans la planche auecla binette, \& le laillerez quelques jours feicher par monceau iur fon gueret, pour par aprés eftre f'cré ea lieu temperé des qualitez de l'air, tirant plutoltaufec, qu'à l'humide.

Pour la Semence, vous choifirez des plus gros qu'aurcz referuées, Sz lis gelées eftans paffées, vous les planterez en bonne terre bicn fumée, \& décliargée de pierres, quict la vraye terre que dafirent lcs Oignons; Vous vous feruirez de voftre Hyuer pour cét effat, en fillonnane la planche où vous le voulez mettre, non en long, mais en traners, \& allés profondément, puis aprés vous polerez vos Oignons au fond de la raye, à vn bon demy pied l'vn de l'autre, \& le recoutrirez en faifant vn fecond rayon, sinfi vin troifiéme, \& vin quatriéme, continuant 


$$
\text { FR A ço Is. }
$$

de mefme jufques au bout de voltre planche.

Quand il eft en graine il eft fort fujet à eftre renucrfé des vents, à caufe de fa charge, \& la foiblelfe de fon tuyau, qui fe rompt ou courbe facilement, lailfant choir fa tefte à terre, qui pourrit la graine au licu de la mcurir: c'eft pourquoy l'on y remediera, en faifant comme vne petite barriere tont autour de la planche, (que j'ay dit aux Salfifix) ou bien mettant des petits ficux d'efpace, en efpace, à chacun defqucls vous lierca quatre ou cing tuyaux, les approchans $\&$ penchans doucement fans les corrompres sil fe peut.

La tige cfant feiche, \& la teftefaifant paroitte la graine à découuert, donne vn témoignage de fa maturité: c’eft pourquoy vous $l^{\prime}$ arracherez, $\&$ a a prés auoir coupé tous lestuyaux, vous mettrez feicher les teftes fur quelque nappe, mettant à part la graine qui tombera d'elle mefme fur la nappe, conme la meilleure, \& la micux conditionnée; puis quand le tout fera bien fec, vous les broyerez dans vos mains, en en retirant auec patience $\&$ à force de feicher, le plus gue vous pourrez.

Si yous ne la youlez broyer à l'heure 
178 LE IAR DINIER

mefme, vous lierez les teftes par bour a quets, \& les prendrezdans voltre ferre: elle fe conferuerá, \& augmentera en bonté, n'en prenant qu'à voltre befoin.

Il y a tant de tromperie à acheter de. cette graine, que je vous confcille de n'en prendre pour femer que de la voltre, fi ce n'eft que quelque amy vous en envoye pour vous renoulueler; car alicuns Marchands vous la vendent trop vieille. \& pat confequent incapable de germer, ou bien ils l'échandent, pour comnoiftre la bonne, il cu fati mettre vae pincée dans quelgue Efcuelle, y metre de l'eaus. \& la faire infuler fur de la cendre clazilde; en per de temps elle pouffera Cors. germe, fi elle eft bonne, finon illa faudra rejetter.

Les Ciboules de toutes fortes, depuis la plus grolle jufques à la Ciuete d'Angleterre, fe plantent de cuilfe, en mettant quatre, ou cing enfemble, pour en faire vne touffer : \& felon la grolfeur de la Ciboule, vous les éloigwerez, n'y ayant. autre foin pour le: gouluerner, que de les. bien farcler, biner, \& fi vous voulez, fumer auant l'Hyuer.

On les pourra, laiffer en leur planche tant d'années que l'on voudra, la plante grofilifant toufiours, par les. 


\section{F R A $x$ ç O I s.}

Caycux qu'elle jette en abondance.

II Cera bon pourtant de trois, ou de quatre ans l'vn, de la releuer, \& de la planter en vin autre endroit; d'autant que la terres'ennuye d'eftre chargée d'vne melme forte, \& s'effrite de la qualité le plus au gré de la plante, ne la rendant que langoureufe, \& debile, fi elle y fejourne plus long-temps.

L'ail fera gouuerné comme l'Oignon, le vraytemps de le planter eft à la fin de Fevrier: le temps de le piler, ou noiier fes tuyaux, eft à la faint Pierre de Iuin: \& le temps de le leuer de terre, à la faint. Pierre d'Aouft; fuiuant le Prouerbe des vieils Jardiniers: A la faint Pierre feme tes Ails, à la faint Pierre pile tes Ails, à la faint Picrre recueille tes Ails.

Quand vous les aurez amalfez, vous les laifferez bien feicher par monceaux fur le gueret: puis à la fraifcheur du matin, votts les licrez auec leurs fueilles mefmes, par douzaines : \& leur lailferez palfer la journée au beau Soleil, a ant que de les porter au grenier, ou en la ferre, les pendans aux foliues du plancher, pour les c onferver plus feichemeat.

Les Efchalotes (ou Appetits) feront ménagées comme les Cibonles, rep!anta nt lsa petits Cayeux pourlesfairegrofo

$$
\text { II } \text { y } j^{\mathrm{j}}
$$


I80 LE I AR DINIER

fir: \& au mois d'Aouft vous recirerez de rerre ce que vous en voudrez ferrer, les mettant en merme lieu que les Ails.

Le Porreau fera auffi ménagé de la mefme façon que l'Oignon, \& replanté en rayon au plantoir le plus auant que l'on pourra afin qu'il ayt le blanc plus long: mefme il ne faudra remplir le rayon que quelque temps aprés, jufques à ce qu'il foit grandy, cela alongera encor le blanc, l'on y fait vine autre façon de plus, qui eft, que quand ils font au bout de leur aceroilfement, on les couche dans leur rayon, les vns fur les autres, ne leur laiffant fortir que bien peu de la fucille, ce qui eft dans terre blanchy; \& par ainfivn Porreau eft alongé de beaucoup, \& fait autant de profit que deux autres.

Pour la graine, vous referuerez des plus beaux \& des plus longs, que vous replanterez au renoutueau : \& quand ils feront montez, vous les entourerez de Pieux, \& Palliffades comme les Oignons, de crainte que les teftes ne tombent à Terre.

Quand elie fera meure, vousla couperez, feicherez, ouferrerez, par bouquets, ou autrement, tout ainfi que J'Oignon.

Les Herbes Odoriferantes, \& autres 


\section{Fr A n çoIs.}

que vous deuez principalement auoir dans voltre Iardin, font celles qui fe mettent en falades, \& dans les apprefts de cuiline; lailfant les autres à voltre volonte, comme Souchet, Bafilic, Lauan* de, Garderobe, Yfoppe, Steca, Meliffe, Camomile \& autres : nous traittcrons icy feulement de celles dont vous ne deuez eftre dépourueu.

Pour les Salades: le Baulme, l'Eftragon, la Perce-Picre, le Crefion Alenois, la Corne de Cerf, la Pimpinelle E- la Trippe Madame, font celle quel'on y employe d'oreinaire conjoinotement auec celles que j'ay décrites aux Sections precedentes, la Salade eftant tant plus agreable, qu'il y a de diucrfitez d'Herbes qui la compolent.

Aucunes de ces Herbes fe fement \& autres fe plante de racine, quoy qu'elles portent prefque toutes de la graine, mais non fi valeureufe que le plant enraciné.

Cilles qui fefoment, font la Corne de Cerf, la Pimpinclle, \&z le Creffon : les autres fe plantent auec racine, \& toutes fe gardent fort bien dans terre, y palfant l'Hyuer.

Vous pourrez les laiffer tant d'Années que vous voudrez au lieu où vous les aurcz femées \& plantées, n'y ayant autre 
38. LE IA R D I IF I R

foin que de les biner, \& en labourer les fentiers, de crainte que les mefchantes herbes ne les eftouffent.

Les autres qui fe portent d la cuifine: font le Thym, ha Sarricte, la Marjolaine des deur efpeces, la Saulge aufí des deux elpeces, \& le Rofmarin toutes lefquelles plantes font aflez faciles à faire venir, \& vous en fournir plus que fuffilamment.

La Regalile ne Cera oubliée, pour la neceffité de ceux qui ont befoin de boira de la Prifane: mais fi vous en metrezdans. voftre Iardin, placez-la en iicu où elle ne vous puiffe nuire; car fi elle trouue $l_{2}$ terre à gouft. elle fe trainera \& profondera beauconp plus que ne fait le chiendent, vous donnant grande peine à la chercher, en cas que vous la voulufiez. entierement arracher.

Elle vient aufi bonne par toute la France, que celle que l'un apporte. d'Efpagne.

Pour vous en fournir, vous prendrez du plant enraciné, que coucherez dans. terre à quatre bons doigts de profondeur, \& vous ne la labourerez point : mais la: bincrez, \& farclerez feulement pour la faire mieux profiter.

Le Thim fe feme \& fe replante de plant. enraciné d'vne touffe en faifant plufieurs. 
brins auec racines, éclattant feulement le pied: \& ille faut planter auec le plantoir large, ainfi que l'on fait toutes fortes de bouttures.

La Sarriette fe feme tous lesans, c'eft pourquoy il faudra eftre foigneux d'en recueillir la graine, \& l'Herbe eftant. feichée, fera conferuée pour les affaifonnemens.

De la Marjohine, il y en a de fran. che, \&d'Hyuer, la franche, ou petite, eft fort tendred la gelée : partant, l'on fera foignaux d'en ferrer la graine, pour en refemer tous les ans: celle d'Hyuer ou la grande, fe pourra perpetuer en quelque lieu que vous voudrez.

Les Saulges franches, \& baltardes, re. prennent parfaicement bien de boutures, \&z de branches éclattéss de leur fotiche anec racines.

Le Rofmarin fe plante aufli de boutture, \& de plant enraciné éclaté de fa fouche.

Le Fenoiiil doux, \& l'Anis, qui font plantecqui fe fement \& gousernent fans beaucoup de foin, ne feront oubliez. en voltre Isrdin.

Contentez-vous du peu d'infruction que je vous donne de ces plantes Odosiferantes; la crainte que j'ay de grollix 
I84 LE IARDINIER

par trop noftre Volume, a fait que j'ay palfe affez legerement par deffus, il refte maintenant pour conclurre ce Traité, d'y adjoufter quelques plantes \& Arbuftes portans fruit, neceffaires à voltre Iardin.

Les fraifes font de quatre fortes, des blanches, des grolfcs rouges, des Capprons, \&ides petites rouges ou fat:uages.

Pour ces dernicresqui font les petites, vous ne vous en metrez beancoup en peine fi vous cites proche des bois oil ellcs abondent : car les Enfans des Villageois en apporternnt alfez, pourucu que vous leur en donnicz quelque peu de chofe: \& fil vous efticz chloignez de ces petites douceurs, vous en pourrez faire de petits Tápis fort eftroits dans le milicu de quelques vnes de vos allées, fans aucun foin ny autre peine que de les planter, en voyant feulement querir du planten mote, où vous fçaurez qu'il y en aura, ou bien de les femer, qui eft de jetter deffus ce tapis l'eau dans laquelle vous aurez laué vos Fraifcs auant que de les manger.

Pour les grolfes Fraifes blanches, rouges, \& Capprons, vous les planterez par planche, quatre rangées à la planche, \& 


\section{F I A C ÇO Is.}

chaque planche à vin pied Se demy l'vne de l'atutre: le meilleur planteft de prendre des trainalfes qu'elles font durant l'Etté, \& en mettre trois planies dans chaque trou que vous fercz anec le plantoir. Le vray temps de le planter cft dan 113 ie mois d'Aouit, lors que cis trainafles font fortes, \& ont pris Racines par les noủds, formans à chacun vne petite planche.

Pour leur guuernement, on les labourcra, farclera, \& binera fort foigneulement.

Pour anoir du plus beau fruit, \& du pius net, l'on mettrra à chaque plante vn petit paifleau, y liant doncement les montans de chacune plante auec du foarre: cela fera qu'outre que le Fruicen fera plus gros, les Limats, Crapaux, Grenoiiilles, \& autres animaus nuíibles ne s'y logeront pas: ce gu'ilsferoient, fila piante rempoit par terre: \& en mangeroicnt vae bonne partic, attaquans toûjours les plus belles.

Au temps qu'dles jotent leur trainalles, il les faudra chaftrer curiculement, n'y en laiflant aucunes, que celles que vous deftinez pour en auoir du plant.

Vous en firez tous les Ans quelques 
186 LE IAR I I IER

noutuelles planches, ruinant celles qui auront plus de quatre ou cing ans, comme commençans à déchoir de leur bonté $\&$ groffeur.

Ii fera bonde les fumer de petic fumier vu peu auparauant les grandes gelées, afin de les ameliorer, coupant toutes les fueilles, comme j'ay dit à l'Ozeille.

Pour la terre qu'elles defirent, le fablon leur eft plus à gouft que la terre forte: C'ctt pourquoy vous chefirez la partie de voftre lardin la plus. fablonneufe pour les y loger.

Si vous voulez anoir des Fraifes dans l'Afrtumne, vous n'auez qu'à couper toutes les premicres fleurs qu'clles poufferont, \& les empefcher de fructifier: elles repoufferont d'autres fleurs par apres, gui porteront leur fruit en arriere-faifon:

Des Framboifes, il s'en troutue de deux coulcurs, de blanches, \& de rougis; elles fe plantent de plantenraciné éclatant vne fouche en plufieurs brins, \& lis plantant à quatre doigts l'vn de l'autre en rayon ounert, qui eft la hauteur du ferd'vne befche, comme j'ay ditcydetrant, quand jay traitté de la maniere de drefier la Pepiniere, où je vous ren- 
F R A H C

raye pour plus abreger.

- Il n'y a autre foin outre les labours, que de tailler le bois mort, \& ofter les jets qui poufferont dans les fentiers, \&r entre deux rangs. Si vous voyez poursant qu'à force de pouffer ils s'eftouffaffent, vous les fuulagerez cn coupant les noutueaux jets, \& laifferez les vieils, comme les plus francs \& capables à porter fruit.

Les Grafeilles font de deux efpeces, des grofies \& petites blanches qui ont des piquerons.

Frd'autres rouges, blanches, \& perlées fans picquerons, que l'on nomme en Normandie des Gadelles.

Elles fe veulent toutes planter \& gouuerner comme les Framboifes, 'c'ett pourquoy je ne $m^{\prime} y$ arrefteray pas d'auantage.

Les Champignons, \& toute autre ofpece femblable, que les Italiens appellent d'vn rom commun à tous Fongi, nolis les diftinguerons en noftre Languie, en lesnommant Champignons de bois, qui font ceux qui viennent à la rive des forefts qui font trés-larges: Champignons de Prez ou paftures, qui font ceux qui croilfent où le beftial paift ordinairement, \& ne pouffent gucre qu'aprez les pre 
I8 LFIARDINIER niters brouillards d'Automne : ce font ceus que j'eitime les meilleurs, tant a caufe de la bcauté de leur blane par deffurs, que de leur couleur vermeille par dcffous : outre ce, ils font encor de fort bonne odeur, ce que n'ont pas les autres. Les Champignons de Iardin qui poullent ordinaireraent fur les couches: \& les moufferons quui ne viennent qu'au com. mencement du mois de May, dans les bois cachez fous la moulfe, d'où ils empruntent leur nom de moulferons.

De toutes ces efpeces il n'y a que celle fur conche que vous puificz faire venir dans voltre Iardin: Et pour ce faire on dreffera vne couche auec du fumier de Mulet, ou d'Aline, en mettant deffus quatre doigts de menu fumier dit terras: \& aprés que la grande chaletit de la coulche fera paffée l'on jettera deffus toutes les épluchures, \& l'eau où l'on aura laué ceux que l'on appreftera à la cuifine: mefmement tous les vieils, \& mangez de verts, ou limats : cetse couche vous en produira de trés bons, \& en fort peu de temps: Cette mefme couche vous pour ra feruir deux ou trcis ans, \& ferabonne à en faire d'autres.

Si vous jettez de cette eau des laucures furles couches à Melons, elles vous 
en pourront produire aulfi: je me fuis laiffé dire qu'il y a des pierre qui eftans mifes dans le fumier one la veriu que d'en produire en fort peu de cemps, \& qu'il $y$ a des curieux qui ont de ces pierres, je $m^{\prime}$ en rapporte à l'experience qu'ils en ont faite. Pourlesmorilles, Etruffes, il $n^{3} y$ en a qu'en certains lieux où elles vienneut saturellement.

Ie croy vous auoir enfeigné tout ce qui fe peut culciuer dans les lardins, au moins ce qui fe mange le pius communement en noltre France Parilienne: les autres Proninces ayant dautres plantes dont ils nous doment les defpouilles à î bon marché, qu'elles ne valent pas la peine de les cultiuer: comme parexemple, les Capres, ce n'if pas qu'elles ne viannant to t bien en ces quariors - cy: mais elles font embarralfantes, occupars vin grand lieu pour peu de rappor : 比 venins mieux entre les pierres de quelque vieille mazure, qu'en tout outre lieu: c'eit vne trop grande peine de s'alfujettir à en cueillir les fleurs pourles confire au fel, \& elles vous reuiendroient à beaucoup plus d'argent, que vous neles acheterez chez les Efpiciers.

Finillons ce Traité, \& monfrons à prefeno la maniere de conceuse en 
190 L E I A R D I N I E R fon naturel, \& confire tout ce que vous recueillirez de voftre Iardin, felon l'ordre des Sections, \& des Articles, comme vos Fruits, Herbages, \& Legumes font en leur rang dans ces deux premicrs Traittez.

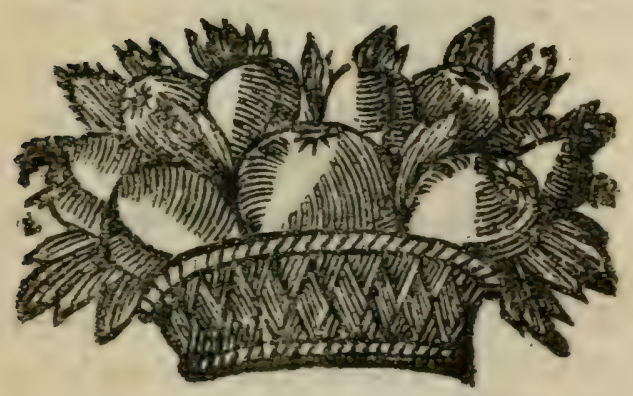


थै uै

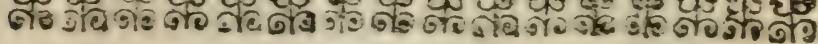

L E

IARDINIER

FRA NCOIS

j

III. ET DERNIER TRAITTE'.

SECTION PREMIERE.

De ls maniere de conferwer le: Frits en leitr narabel.

T In y a ricn qui touche plus viuemenc les Sens, que d? voir dans le profond de l'Hyuer des Fruits aufí beaus \& bons, voire mefme meilleurs que quand on les à cucillis. Et alers qu'il femble que les Arbres foient morts pour ne plus reuerdir, \& que par la rigueur du froid voltre Iardin eft tellement dépoiiillé de tou t ce quil l'embellilloit, qu'il paroift plutolt vn defert qu'vin lieu de de. lices: c'eft alors que vous gouftez vos 
192

\section{LE IARDINIER}

Fruits auec beaucoup p'us de contente. ment que dans l'Efté, ou par la trop grande quantité \& dinerfité, ils vous font plutoft à dégouft qu'agreable. C'eft donc pourquoy il nous faut effayer de vous monttrer les moyens les plus certains, \&: les plus faciles pour les bien conferuer tout le long de l'Hyucr : \& mefmes juf ques à ce que les nouueatix vous incitent à quitter les vicils, car il eft des fruitis comme des Vins : ceux que l'on boit les premiers, font les plus delicats, \& les plus feuez; \& ceux que l'on garde pour l'arriere - laifon font les plus fermes ou rudes, neant-moins dans leur temps trés. agreable : mais fi tolt que les nouucaux fent frais, \& en boitte, l'on quitte les vieils, qui auparauant eftoient eftimez trés excellcnts: De mefme auffi, fi tolt que les nonueaux, Fruits approchent de leur maturité, l'on abandonne ceux de l'Année precedente; \& vn plat de Fraifes, de Cerifes, (quoy que toutes vertes (ou de Poires de Haftiueau, fera preferé à la meilleure Poire de Bon-Chreftien. que vous ayez.

Pour paruenir à noftre intention il fera bon de choifir quelque licu dans voftre logis ani foit commode ponir en faire sn Fuidier, ouretertivir, griait lesfine- 


\section{FI A r çoIs.}

ffres \&ounuertures petites a 93 Jée, oruucrurespetites a caufe de la geIéc, \&rdu grăd chaud; lefquellesvours tiendrez toûjours bien fermées, $n^{\prime} y$ laiffant entrer aucun air, \& ne vous en feruant que pous la clairté, refermant tous les vol. lets quand vous en fortirez: mefmement giuand il n'y en auroit aucune, \& que la porte fut petite \& balfe : ce feroit encore le meilleur, la tenant fermée fi toft que yous ferez entré dedans.

Le licu eftant dcftiné pour voitre fruitier, vous le ferez garnir d'a ix en tablectte tout autour: en cas que le lieu foit grand, \& que l'on puill qaire dans le milieu des monceaux, ou mijols des Fruits les plus communs, quel on deftine pour les Valets : \& en cas qu'il foit petit, vous vous contenterez d'en mettre de trois coItez, referuant le quatriéme, pour y placer vos mijols.

Ces aix feront pofez fur des Confoles do Bois, ou de fer, bien furtes ì caule de la charge; deux colte à colte, pour faire la largcur de deux pieds : vous y cloiierez rne petice latte par deuant, de crainte qu' en maniant les fruits il ne roulent \& tombent : Vous laiffere totrois pieds de vuide par bas, pour mettre lès petits monceaur des fruits les moins precieux, en les diftinguant \& feparane 
3.9 4 I E I A R D I IER

felon leurs efpeces; \& continuerez des rangs de tablettes, jufques au Plancher d'enhaut, les polant les vnes fur les autres à la diftance de neufà dix poulces.

Pour voltre plus grande commodité, vousaurez vn degré de boisficile à porter, qui leruira à vous élcuer, jufqucs à la plus haute tablette; quand vous vifiterez vos fruits, vne échelle n'eftant fi cómode, 2x laiffant beaucoup plus de pieds, outre le danger quil y a, qu'en vous aduançant \&allongeant par trep pour atteindre par foís en quelgue lisu éloigné, l’efchelle pourroit gliffer ou tourner.

Le temps venu de cueillir vos fruits de garde que vous reconnoitrezen plufieurs façons: Ou quand ils commencent à tomber $d^{\prime}$ eux mémes, ce qui arriue ordinaircment aprés les premieres pluyes d'Automne, quand l'Arbre rafiraichy \& \& humecté, rentle fon bois, \& deftache les fruits; ou quand les premieres gelées vous aduertiffent de les ferrec; ou pour vn temps plus prefix, dans le decours de la Lune d'Octobre (cecy foit dit pour les Poiriers, \&e Pommiers (eulement) commençarit à cueillir les plus tendres les premiers, \& finiffant par les plus fermes pour leur donner plus de loifir qu'aus autres à s'achence de meurir. 


\section{F I A n ço is.}

Ii y a quelques fruits quine fe veulent manger que mols, comme les boires de Grofmenil, les Cormes, Alifes, Azerolles, \& autres: lefquels vous laifferez à l'Arbre tant que vous royés que chcaints cn quantité d'cux-mermes, ils vous obligent à les ferrer.

Pourles Niffles, le vray temps de les cueillir eft à la faint Luc, afin d'obeyr au Prouerbe.

Qirand rous cucillirez vos fruits, if fudra auoir des corbeilles d'ozier bien fortes, pour eltre portées pleincs par deux Homm:s, \& vous mettrez vn peu de foarre au fonds, pour empefcher que le fardeau de celles de delfus, ne froiffe celles de deffous contre la corbeille.

A mefure que vous détacherez vos fruits, vous en ferczélection mettant les gros, les moyens, ceux quifont tombez d'eux mermes, ou que vous aurez abbatus en cueillant les autres, chaque forte en fa corbeille à part. Ie ne parle point icy despetits, outrognomnez, car ie fuppole que vous cn aurez déchargé vos Arbres, beaucoup de temps auparanant $\&$ dés lors que vous aucz reconnu qu'ils ne poumoicnt proficer, alin de domer plus de nourriture aux beaux. 
296 LE I A R D I N I-ER

Les Pommes percées de vers, feronc auffi miles auec celles qui tombent pour pour eftre mangées des premieres.

A merme que vous cueillirez vos fruits, vous les porterez dans voftre fruicticr, \& rangerez les plus precieux; fur vos tablettes, fans qu'sls fe touchent, mettant vn peu de foarre long deffous, diltinguant les beaux d'auec fos moindres, partablettes particulieres, \& mettant les tombez, \& verreux en monceau, ainfi que je viens de dire.

Quant aux Poires de Fon-Chrefticn, elles fe veulent cueillir plus curicufement que les autres: car à celles qui font belles \& bien colorées, rouges d’vn cofté, \& jaunes de l'autre, il leur faudra feeller le bout de la queuë, suec, de la cire d'Efpagne pour arrefter la feve qu'elle ne s'euapore: puis aprés on les enueloppera de papier bien fec, \& Is mettra.ton dans des boilfeatux coliuerts, afin qu'elles prennent vnes belle couleur blonde eftans eitouffées dans ces boiffeaux.

Vous pratiquerez le mefme aux Poires de Double-Fleur, de Cadillac, de Toul, \& autres qui greffees fur le CoiTinaffice prennent couleur de l'Arbre: Cur quant à colles gui font greffies fut 
ranc, elles demeurent ordinairement vertes: C'eft pourquooy fans y apporter tant de peine, il ny aura quà les ranger fur la tablette, comme j'ay dit cydeffus.

Les plus curieux ont vne armoire qui ferme extrémement bien, dans laquelle ils mettent leurs Poircs de Bon-Chreftion, elle eft garnie de tablettes, \& fur chaque tablettes il y a de petites tringles de bois, qui fe creifent en forme de treillis, dont les quarrez font à peu prés de la grandeur qu'vne belic Poire peut eftre grolfe : fur chaque quarré ils y mettent vde Poire à part, de crainte qu'elles ne fe touchent, \& s'il y en a quelqu'vne de pourrie, qu'elle ne gafte la voifine: ils tienment cette armoire toûjours bien fermée, mefmes ils collent des bandes de papier autour des guichets, pour empefcher entierement l'air d'y entrer, n'y regardans que quand ils en veulent prendre, cela les eftouffe, \& lcur fait prendre vnetrés-belle coulcur: mais auparauant que de les enfermer ils laiffent les Poires cing on fixjours dans les corbeilles telles que l'on les a apportées du Jardin afin qu'clics fe refuënt.

Pour les fruits qui fe mangent mols; il les fatat mettre en mijol, \& s'ils ne 
93 LE IADINYET

meuriffent alfez promptement à voftre volonté, vous les metrez dans vn fac à bled, \& les ferez blutter par deux perfonnes; le heurtement qu'ils fe feront les vins contre les autres, les auancera beaucoup de meurir.

Les Raifus mulcats ou autres communs fe conferuent en plufieurs façons, ou en les rangeant fimplement fur du foarre, ou en les pendant à des cerceaux au plancher, \& les courunt par detius auec du papier, pour les garentir de la poudre, ou en les metrani allec de la paille d'Auoyne, ou de la cendre dans des tonneaux.

Ie laiffe icy plufieurs manieres curieufes $d^{2}$ en conferuer, comme, lors que la grappe eft en fleur, In faire entrer dans vn boccal de verre: puis eftant meure la couper, fceller la queuë, \& l'atracher en forte qu'elle pende dans le boccal fans y toucher, \& auffi bien boucher l'entrée du boceal auec de la cire molle, en forte qu'il n'y entre aucun air, cela conferue la grappe jufques aux noumelles.

Il y a encor beaucoup d'autres moyens que je méprife à caufe du peu de prot quel'on tire, \& de l’embarras \& dépenIe qu'il y faut faire.

Quoy que je n'aye pas cy.demant en- 


\section{FRANÇÓ I S.}

feigné, comme il fe faut fournir de mufcats de toutes les coulcurs, ce n'eft pas que je l'ignore, car j'en futs amplement pourueu, mais comme c'of vine plante qui fe veut gouturner ainfi que les autres vignes, $j$ 'en laiffe le foin à mes vignerons, qui dés leur jeuneffe font nourris au trauail de la vigne, l'experience leur faifant connciftre les fujettions neceffaires, que lis Iardiniers nobferueroient pas auec tant de precautions qu'eux : particulierement au planter \& à la taille, qui font les feules façons que je leur fais faire? $\&$ dont je me troune fort bien.

Ie vous diray feulement fur ce fujec que les Mouches de toutes fortes, comme Abeilles, Guefpes \& autres, les Fony. nes, Lers, \& Rats-Verets, font extrémement friands de ce railin quand il eft bien meur : pour y remedier il faut mettre des Ails, à domy échachez en plufieurs endroits de voftre Treille, \& alfez proche des grappes, leur forte odeur les en chaflera.

Le plus grand afpect du Soleil de mi$\mathrm{dy}$, \& l'Abry de quelque muraille eft le vray licu que defirent les mufcats.

Pour conclurre cette Section, je vous aduertiray de viliter foutuent voftre fruisticr, afin que s'il y a quelques fruits qui 
200 LE IA R D I IIER

fe pourriffent que vous les ofiticz, car par leur attouchement ils gafteroicnt les plis fains: s'il s'en trouue quelqu'vn qui foit rongéde Souris, vous ne l'ofterez pas de fa place; car tandis qu'il y aura dequoy manger à celuy.là, elles ne toncheront point aux autres : mais tendez des fourriciers proche de là, afin de les prendre: Car d'y laiffer hanter les Chats, ils dérangeroient tous vos fruits, \&- feroient leur ordures dedans les mijols, \& fur des tablettes.

\section{Des fruits feichez. \\ SECTION II.}

R y a quantité de fruits que nous fei1 chons au four, que l'on feicheroit au Soleil en Pays chaud, comme en Prouence les Brignolles, en Languedoc les $\mathrm{Paf}$ feriles ou Raifins: mais puis que la froideur du climat nous oblige à nous feruir dufour; je particulariferay icy de quelle façon chacunveut eftre feiché.

Commençant parles Cerifes, Guines, \& Griottes, comme par les premiers que que la failon nous prefente; vous les choifrez bien meures, grolles, \& qui ne foicnt point tournées: Vous les polerez 


$$
\text { F.R A N C O I S. }
$$

fur des clayes, lestangeant colte à cofte, le plus proprement quil fe poursa, fans qu'elles foient les $\nabla$ nes fur les autres, y laiflant les queuès \& les noyaux, puis volis les meitrez dans le four; qui fera remperé de chaleur, comme quand on a tiré le Pain d'vne fournéc \& les y ayant laiffées, tant qu'il aura de la chaleur, yous les retirerez, \& remuerez toutes, en les changcant 'de place, afin qu'clles feichent parfaictement; puis vous rechaufferez le four, les y rewettant tant de fois, que vous reconnoiffez qu'elles foient fuffiamment feches, pour eftre gardées: Vous les laifferez refroidir en monceau vn jour entier, par aprés vous les lierez par bouquets \&z les ferrerez dans des quaiffes ou boiffeaux bien fermez.

Les Prunes feront fechées conme les Cerifes, \& ne feront cueillies que trésmeures: les meilleures à feicher font celles qui tombent d'elles-mefmes, carelles auront plus de chair, \& feront plus agreables a manger que celles que vous détacherezde l'Aubre, qui gardent toûjours quelque verdeur.

Pour les plus excellentes à faire Pruneaux, on choift les Imperiales, Dattes, fainte Catherine, Diaprées, Perdrigon, Cypre, Brignolles, Damas de 


\section{LE I A R D I I ER}

tontes les fortes, \& faint Iulien pour le commun du Logis.

Si vous voulcz accommoder des Prua nes à la façonde Brignolles, il vous faut choifr de quelque belle efpece de vos prunes, comme de Perdricon, d'Abricot: moyeu-dœuf, Brignolles, ou autres qui auront la peaublanche; les peler fans coufteau en tirant la peau qui quitcra airement la prune fielle ett bien meure:puis ofter les Noyaux, fans rompre le fruit, eomme i'enfeigneray cy-apres, cn parlant des Abricots; faire bicn bouillir ces peaux auec peud'cau, palfer le tout par vin gros linge; \& dans ce ius qui fera en confiftance de Syrop, y iremper vos rrunes à chaques fois que vous les mettrez au four, en les applatiflant autant de fois: fi voltre ius n'eft afrezen Syrop, vous prendrez du ius de Gadelles blanches, bien meures, cela rendra voftre Syrop fort efpois, \& bien lié.

Les Prouenceaux au lieu du Four, les mettent à des branches d'Efpine., à chaque piqueron vne, \& les lailfe ainfí feicher au Soleil.

Les Pefches s'accommodent de la mefome façon que les Prunes, mais il les faut cueillir à l'arbre; car celles qui tombent, outre ce qu elles font trop meures, 
elles fe font des meurtrilfeures qui ne fecheroient qu'à grande peine, \& feroient defagreables au manger : auant que d'ofter les noyaux, vous les mettrcz vne fois aufour, pour lesamortir, puis vous les fendrez proprement auec le coufteau, tirerezle noyau, \& les outrirez, \& applattirez fur quelque Table, afin que lesmettant au four elles fe fechent aufia bien par dedans que pardehors, à caufe de leur grande efpoiffeur; \& au dernier coup qu'elles fortiront du four eftans encore toutes chandes; vous les refermerez, \& applatirez pour leur redonner leur premicre forme.

Lcs A bricots ferontaufí cueillis à l'arbre bien meurs : il ne fera befoin de les outurir pour leur ofter les noyaux : mais feulement les pouffer proprement par l'endroit de la queuë \& ils fortiront par le bout: En les fechant l'ont ne les oviure pas comme les Pefches, mais on leslaiffent entiers, fe contentant de les apla. cir, a fin qu'ils fechent efgalement, \& qu'ils s'arrangent mieux dans les bcëtes. Les Poires fe fechent pelées \& fans peler, tout de mefme maniere que i'ay en * feigné cy-deuant : eftans pclée, elles en font beancoup plus delicates, \& l'on fe fert de peaux pour les tremper dass le 
10.4 LE IARDIN IE ius, ainfi que ía dit aux Prunes pelécs: on leur laiffe la queu \& la tefte con les pelant : il ne gift qu'à faire eflection des bons fruits les plus delits \& les plus mufquez, comme lOrenge, \& le bonChreftien d'efté, la Mufcadille, le gros Mufcat, le Rouffellet, le Bezy d'Airy, \&cent autres qui fe trouticiont excellens.

La Poire ne veut pas eftre cueillie trop menre, car cela la rend mollatie.

En vendanges, vous pourrez metre parmy vos pefures du vin blanc doux a lieu d'eau : \& auffi quand fe fera la faifon des Cydres, y metrre du loiré doux, fait fans cau.

Les Pommes fe fechent ordinairement fans peler: \& fe coupent par moitié, oftans le trognon: Vous pourrez en fairs boüillir quelqnes-vnes pour en tirer le ins afind'y rempercelles que vous volidrez fectier.

Les Raifins de toutes fortes, Mulcats \&autres, feront aufi fechez au four fur la claye: n'ayant autre fuiection que de ne leur donner trop chaud \& les retourner louuent: afin qu'ils feichent éfgalement: Les Langnedociens les paffent dan. la lexive, auant de les feiclicrau Soleil. Ie mettray auffi au rang des fruits fecs les Feves vertes, lefquelles bien appre- 
ftées, auec quelque peu de Sarriete fiche, qui eft le vray affaifonnement des Feves, pafferont pour nomulles.

Pour les feicher, wus prendrez des Tendresqui ont encore la peau ou robbe verte, \& non pasblanclics: vousleur ofterés cette robbe ( ce gue l'on appclle à Paris fraizer) puis les mettrés Ceicher au Solcil fur despapiers, en les remuant pluficurs fois le iour : vous les retirerez le foir dans le logis, \&z les remettrez rous los iours au Soleil, iufques à ce que vous reconnoificz qu'elles feront biew Ceiches, quilera cn peu detemps, fi le Soleil n'eft couvert de nuées : Eltans ieiches, on les lerrera dans des Boifieaux counerts, les gardant de toute humidité.

Il les faudra lailfer tremper, ou infuferdans l'eau ticde, entiron demy iour auparauant que de les mettre cuire.

Pour les Pois Teits, on choilira des plustendres: \& tirés de leur colfes feront mis ficher comme les Feves : auant que delescuire, vous les metirés tremper en eau tiede, adinufrant à leur cuiffon vae poignée de fucilles de pois nouncaux, fi vous en aués en vert : 1 i int auec du fil, de crainte qu'elle ne fe melle auec les Pois.

Les Morilles \& Mouferons feront en: 
206 LE IARDINTER

fles, \& pendus in grelque licu al la chas leur, comme le defiusd dun four, oì la ils feicheront facilement: \& fir vous n'auez aucun lieu commode, il fuffira de les prendre deuant le fu, ou mettre dans le four moyennement chavd.

Des Confiures an Sel, of Tineigre.

$$
\text { SEC T ION III. }
$$

Es Concombres, font les plus gros Lfruits du Iardin potager qui fe confifent au Sel:ou les prend ou fort petits (que l'on appelle Cornichons, à caufe que lon choifit d'ordinaire ceus quifone crochus; dautant qu'ils ne proficent point en groffifiant:) uu déja gros, mais tendres, nayans encore les pepins durs, car ils feroient defagreables au manger: Ceur-cy fe confient pelés ou fans peler; mais il vaut mieux les peler auparauant que de les confire, qu'apres qu'ils font confits, à caufe de la perte du Sel \& du vinaigre qui aura confit cette peau, qui pour fa dureté n’eft pas facile à manger: ils font auffi plus propres \& plus blancs, eftans pelezà l'inftant que l'on les veut feruir, que ceux que l'on pele auant que de les confire, fi bien que vous en vie. 
FRANÇOIS. $\quad 207$

rezcomms v us wudrez.

Les autres, yoi font les Cornichons, fe confil ur fanspeler, à caule de la delicateffe de leyr peau.

Guslescucillite dés le matin par vn beau comps, leur lathant paffer la iournée au Soleil, pour les amortir rn peu, afin qu'ils prennent mieux leur lel.

Vous mettrez les pelez, ceux lans peler, \& les Cornichons chacun à part dans despotsdegreis; (car ceux de Terre re pourrillent par laforce du Sel qui les penetre, perdant leur fanmure, it vous les arrangerez proprement, les pieflant le plus que vous pourrez, fans les froilfer: vous ietterez pardeffus du fel cin bonne quantiré, puis du vinaigre, infques à ce que ceuxd'enhaut trempent autrement il s'y feroit one moifflenre, qui gafteroit ceux qui ne tremperoient pas: Cela fait, vous les ferrererez en lieu tempeté de chaud \& de froid, n'y toulchant de fix femaines au moins, afin qu'ils fe confifent parfaitement : voltre fruiticr fera $\tau \mathrm{r} \in \mathrm{s}$-propre pour les bien conferuer.

Le pourpier que vous confirez, fera pris de celuy que vous aurez replanté, pour céreplus beau \& plus gros: Le vray temps pour le cucililr, c'ct quand il commence à flcurir, fi yous voulez l'auois 
208. LE IARINIEIR

bien tendre: car if vous atcendiez qu'il fut défleury pour ausir lagraine, (comme l'on le vend ordinairenent, ) il (eroit trop dur à manger : vous le ferez amortir ou deffeicher denx on trois iburs au soleil, puis apres wis le rangerez dans les pots de grets, le fallant \& couurant de vinaige ecommeles Concombres

Les Capres, les Eleurs de Geneft, Ia Perce-Pierre, l'Eftragon, \& autres Herbes femblables, ferone aufi confites de la mefme manicre que les Concombres \& le Pourpier.

Les fonds d'Artichaux fe confifent au fel, mais d'vne autre methode que les precedens, car ils veulent eftre cuits plus qu'à demy : \& cftans refroidis \&z bien efgoutez de toute leur cau, mefmement effuyez auee des linges pour n'y zuoir plus d'humidité : l'on les rangera dans les pots, 2 l'on refpandera deflits de l'eau fallée autant cu'clle le peut-citre, c'eft à dire, que pour les rondre iufques à ce point, il y faudra mettre du felautane qu'elle ne pours fe fondre, \& ce iufques à ce que vous reconnoiffez qu'erz y en mettant dauantage, elle n'en puiffe plus fondre, \&e que le fel aille au fonds en fon enticr; cette eau fe nomme eats: Marinée. 
Par deffus cette eau qui furnagera vos Artichanx, vous conlerez de bon boure fondu la hautcur de deux doigts, afin que l'air n’y puilfe entrer, puis le beure eftant bien refroidy, vous les ferrercz auee les Concombres, ou en lieu femblables, \& coutrirez de telle façon, que les chats \& fouris n'aillent manger le beure.

Ie prefupofe que vous les ayez preparéa avant que deles mettre dans les pors; de la melme façon que quand on les veut feruir fur table, qui elt d'ofter tout le vert de leurs fueilles, 8 le foin de dedans.

Le vray temps pour ce melnage eft en Automne, quand, cn pratiquant ce que i'ay dit cy-decuant dans le ficond trairé, au chapitre des Artichaux ] vos planies vous en produilent de tendres : ee font ceux-là que deuez prendre pour faller, \&e vous nattendrez pas quils s'onurent pour fleurir, mais vous les prendrez quand ils font encore en pommes bien formées.

Quand l'on en voudra manger, on les deffallira enticrement, lcs changeant d'eau, \& les remettra- on boilillir encorev ve fuis auant que de les prefarer pour la table. 
2TO IE IARDINIER

Les Afperges, rois fans Colfe, Morilles, Champignons \& Moufferons, feront auffi confits au fel, [ les ayant fait cuire à de. my, \& apprefter chacun felon [a façon] de la mefme maniere quéles Artichaux.

Vous vifiterez au moins tous !es mois vos pois, afin que s'il y anoit quelque chofe de chancy, ou que quelqu' rn perdit fa fammure, vous y apportafliez remede.

Ie me fuis aduilé, il y a déja quelques années, de confire des Cormoviilles, \&r les ay fait palfer pour des Oliues de Verone, à beaucoup de perfonnes qui sy font tromnies, la couleur eftant toute femblable, \&i le goult peudifferent : Ie fais careillir les plus grolles, \& mieux nourries, au temps qu'elles veulent commencer à rougir : les ayant lailfé $v$ in petit fannir, ie les mets dans des pots ou barils, ¿x les emplis d'eau fallée tout femblable à celle que iay dire pour les Artichaux : \& pour leur donner bonne odeur, $i^{2} y$ adsoufte du fenouiil vert en branche, \& du laurier, puis ie bouche bien le vailleau, \& n y tonche de trois mois apres. Si clles ont pris trop de fel, on lesdeffallera auparauant que de les feruir fur la table. 


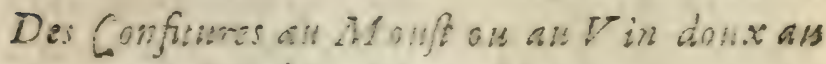
Cidre, o aи Miel.

\section{SECTION IV.}

T Outes fortes de fruits qui fe confiSent au fucre, fe peuuent aufliconfire an mouft, au Cidre, \& a miel; \& il $n^{\prime} y$ a autre fujection à choifr ceux qui fe veulent porboivilir auparaunt que de les confire, d'auec coux qui ne s'euerdument point, que celle que l'on feroit fi lon les confifoit au fucre: C'eft pourquoy i'aime mieux me retenir danscerte fection de vous les dittinguer, attendant à vous en donner vne incelligence tresample, en la fection fuiuante, ou ie m'etendray tout au long, vous enfeignant les diucrfes manieres de faire des Confitures liquides; en enfuiuant !'ordre \& le rang des fruits que iay tenu dans tous les Chapitres de ce liure, pour ne ricn laiffer palfer fans vous fatisfaire, \&e vous rendre capables de confire tout ce qui fe recucillera de voftre Iardin propres aे Confitures.

Pour defcrireicy feulement les principales fuicctions que l'on doit obferuer neellairement à confire le fruit au arount ou 


\section{I2 LE I A R D I N I E R}

vindoux, vous prendrez trois feaux, ous trois pots, outrois parties du mouft, felon le peu ou le plus que voulez Confire de fruit : vous les mettrez dans vn Chatidron ou Poëllon fur le feu, \& prendrez garde li vous faites voftre feu auec áu bois, que la flambe, pourctre par trop grande, ne brulle voltre Chaudronné par quelque colté. Vous-ferez boüillir ce Moutt iulque à la reduction de trois à v $n_{3}$ afin qu'il ne s'efpoiflite \& puifre confire fuffiamment voltre fruit, pour efte de garde fans fe chancir ny gafter.

Vosfruitseltans pelez, ou fans peler, felon leure elpeces, ou la delicatcileque vous y. voulez apporter : Ceux qui doiuent eftre parboüillis l'eftans, \& bien efgouttez \& fuichez de toute leur eau, fe- ront ietiez dans ce mou?t, \& confits, les efcumant foigneufiment, \& fairant boüillir iufques à ceque volis voyezque le firop foit en bonne confiftance, ce qui fe cónoilt en en mettát quel ques gouttes fur vne Affette ellesdemeurent en rubis, \& ne coulent point en penchant l'Affiette-

Le ronft ne tçauroit eftre pris trop doux, c'eft pourquoy, fi toft que l'on ap. portera des grappes bien meures de la vigne, vous les ferez promptement fouler, Ex prendrez de ce mouft ce qu'en au- 
Fee de befoin, blanc, ou rouge, felon ce que vous voudrez confire : aucuns fruits, comme les Coins, les oires, les Raifins ronges, \& femblables, veulent que ce mouft foit de grappes rouges : autres le veulent de grappes blanches, comme les Noix, les Railins Mufcats, \&r attres à qui on defire conferuer la blancheur

Aus: fruits que vous confirez, oil it faudra du vin rouge, mettez y pour releuer le gouft, la Canelle, \& le Cloud de Giroffle, les enfermani dans vn perit noüet de linge, afin qu'ils ne fe mélentauec les Confitures \& fe perdent ou confomment dans le Syrop: Et à ceux qui voudront le vir blanc, vous n'y mettrez que le fenouiil vert. l'enfermant aufi dans vn linge.

Le Raifinè fe fait en prenant de belles grappes rouges des plus meures, \& ne lescueillans que laprefdifnée au Solcil, afin que conte l'humidicé en foit bannie. Vous.les mettrez en queligue grenier de voftre logis, oû l'air \& la chaleur donnent aifement, les eftandans fur destablesou clayes, pour là les laiffer quinze jours au moins fe reffuyer \& amortir ; à faute qu'il ne falfe beaucoup Soleil, ou que le temps foit froid, rous les mettrez vi peu aufourb:en remperé de chalcur: 


\section{I4 LE IAR DINIEI.}

Apres quoy vous les prefferez dans lis mains cn leur oftant toutes les Rafles, ou Rafteaux, \& mottrez peaux \& ius boiil lir dans le chaudron en l'efcumant foigneufement, \& oftant le plus de pepins que vous pourrez : vous ferez reduire le tout à la troifiéme partie, diminuant le feu à mefure que voltre Raifné s'époifit, $\&$ le monuant foument auec vne gafche ou cucillicr de bois, de crainte quil ne s'attache au Chaudron, St pour le faire cuire également : eftant reduit, vous les palferez autraners d'v ne cftamine ou gros Linge, froiffant les peaus auec la cueillier de bois pour en exprimer toute la fub. ftance : \& de plus vous les preiferez en tournant l'eltamins ou auec des preffes; Ccla fait vous les remettrez encore fur le feu, \& les recuirez, tournant incelram. ment tant que vous iugiez voftre $R$ aifné eltre allez cuit; puis vous de tirerez du fen, le verfant dans desterrines, de crainte qu'il ne contracte quelque mauuais gouft du chaudron : eftant demy froid, vous le nettrez dans des pots de greis, ou Fayence pour le conferuer.

Vous lailferez vos pots defcoutuerts cing ou fix iours : puis vous y mettrez vin papier, tout de la rondeurdu dedans de voltre pot, lequel touchera par tout à 


\section{FRA}

roftre Raifiné : \& quand vous vifiterez vos pots, G vous royez que voftre papier foit chancy, vous le leuerez, y en remettant vnautre; \& ce tant de fois que vous y verrez du moirí : qui fera iufques à ce que toute l'humidité foperfinè foitéunporée : alors il ne chancira plus, ficen'e?t que voltre Raifiné ne foit pas allez cuit: auquel casille faudra recuire, puis vous le counrirez à demeurer.

Pour faire de la monflarde à la mode de Dijon, il ne faudra que prendre de ce Raifné, \& y mettre de la graine de Senevé mouluë ou broyte dans vn morticr, pris paffée au Tamis bicn delié: eftant bien meflez enfemble, il faudray efteindre des charbons ardents pour ofter l'amerume du Senevé : puis la mettre dans des barillets ou pots de grots que vous boucherez bien, \& les ferrerez pour voltre vfage.

L'on confit aufifi toutes fortes fe fruits zu Cidre de Poire fait fans eau, en le faiant reduire au tiers par l'ebulition, de a mefme façon que íay dit au mouft. Pour confire au tniel, vous prendrez iu plus efpois plus dur relfemblant u Sucre, \& le ferez boüillir dans vne oefle à confitures, l'efcumant bien cuicufement, \&remuant de crainte qqu'il 
216 LE IARDINIER

ne brufle : pour voir quand il fera cuit, faut prendre vn œufde poule, \& le mettre fur voftre miel, s'il enfonce il n'eft pas affez cuit, \& sil flotte deffus, ilfera en bonne confiftence pour confire ros fruits: Vous fçauez que le miel eft fort aifé à brufler : c'eft pourquoy vous acheacrez voftre cuiffon à petit feu, remuant founent le fond de voftre chaudron auec voftre gafche ou cuillier, de crainte qu'il ne brufle.

De: confitures liquides au Sucre.

\section{SECTI ON V}

Coy que ie deurois icy commencer par les Amandes Grozeilles, Abricots verts, \& fruits rouges, comme les premicrs qui fe prefentent pour eftre confits, neantmoins i’ay cltimé qu'il valoit mieux fuiure l'ordre que nous auons déja tenu, que d'entre-mefler les articles, où par auanture $i^{\prime} e n$ pourrois oublier quelqu'vn, ce qui vous donneroit du mefcontentement-

Nous commenceront donc par les Poires, comme celles à qui i'ay donné le premier rang, \& diray que chezles Confifeurs de Paris, il ne s'en troule que de 


\section{FR A N ÇO Is.}

quatre ou cing fortes qu'ils gardent, ou liquide pour faire fechér à mefure qu'ils en ont befoin pour lé debit : Ces poires font la mufcadille, le gros mufcat, le Blanquet, le petit Rolfelet, \& l'Orenge, neantmoins toutes fortes de poires le peutent mettre au Sucre: vous preferercz pourtant lcs fruits delicats ceux qui font fermes.

Pour paruenir donc à noftre intention vous pelerez proprement vos fruits, faifant la peau la plus deliée que vous pour$\mathbf{r} \in \mathbf{z}$, \&z leur ofterez la tefte, laifiant la quené entiere \& ratillée aux plus petites : \& vous couperez les plus gios par moitié, leur oftani le trognon, \& ne leur laifran qu'vn bout de queue : A mefure que vous les pelerez vous les ietterez dans l'eau fraiche de crainte qu'elles ne moirciflent: en apres vous les ferez parboiillir ou éuerdumer pour aider à la cuifon, \& rendre plus preparées à receuoir le Sucre, puis uous le tirerez de l'eau \& les efgottcerez fur quelqueClaye: cela fait rous les peferez, \& mettrez aus moins autant de livres de bon Sucre qu' il y aura de livres de fruits : vous callerez volte Sucre, en forre que le plus gros morceau n'excede en groffeurvne Cha. ftaigne : vous mettrez voltre fruit dass 
218 LE IARDINIER

la poëlle \& poudrerez valtre Sucre pardeflus, y ietrant vn peu d'eau pour aider à le fondre feulement : apres vous mettrezvoftre poëlle fur le feu, \& confirez voftre fruit le plus promptoment quo vous pourrez, afin que le bouillon furnageant continuellement le fruit, il le cuife également.

Vous aurez toûjours l'Efcumoire à la main pour changer voftre fruit de place, \&. efcumer continuellement : vous ne leuerez voftre poefle de deffus le feu, que vous ne iugiez voltre fruit entre confit : ce que vous pourrez remarquer par le bo illon, qui s'abbaiffant ne fera tant de mouffe ou de boutteilles qu'au commencement, \& par les gouttes de Syrop (que vous mettrez fur vne affiette, ) fi elles ne coulent point : alors yous tirerez voltre poëfle de delfus le feu, \& verferez vos confitures dans quelque terrine, de crainte que feiournans dans la Poëlle, elles ne contractent quelque mautais gouft, prouenant du cuiure : vous les laifferés trois ou quatre iours repofer dans cette terrine en lieu exempt de la poudre fans les courir, afin que toute l'humidité dufruits'éuapore, \& que le fruit prenne Sucre : au bout defquels, vous pancher६z vot teretrine, \& s'il y a quelque eau 
guifurnage le Syrop, vous l'efgoutterez, puis renuerferez vos conficures dans voAre Poëlle, afindeles faire recuire fi ellesen ont de beioin, \& les laiflerezvr peu refroidir, auant que de les nettre dansles pois pour les ferrer : vous les laillerez quatre ou cing iours fans les couurir: au bout defquels, s'il y a quelque humidité dellus vous l’efgouterez : puia vous les couurirez commeiay dit cy deuant, y metrant vn papier de la rondeur du por qui touche le syrop : Er fivous lestromiezaliez cuittes du premier coup apres les auoir efgouttées; vaus vous contenterez de les faire chaufiter fans les vuider de la terrine, en les meflant auec la gafche ou l'écumoire, \& cftans vn peu refroidies, vous les metrrés dans les pots, comme ie viens de dire.

L'on confit alifil des Poires \& $\&$ autres fruits pour eftre mangez chauds, ce que lon appelle Compoftes : comme par exemple le petit Certeau, \& autres felon la conftume du pay sou vous ferez.

Pour lesconfire, vous les pelerez,comme íay dic cy-deffus', Si les metrezdans in poidecerreneuf: à vne liure de poires, il fiffit d'vn bon quarteron de Sucre, auec la moitié d'rn demy-fepicic de bun vin vermeil, \& de l'eau, iufques 
220 LE IAR D INIER

à ce que celles d'en-haut trempent : vours les couturirez du couuercle, \& les ferez boüllir à feu mediocre, les remuant de cempsentemps, pour ne les laifler atta * cher au pot: \& quand elles feront à demy euittes, vous y mettrez de la Canelle \& du Clou de Giroffle, les acheuant de cuire à perit feu, iufques à ce que le Sy. rop foit allez fait à voftre volonté.

Pour lesferuir fur table, vous les rangerez le plus proprement que vous pourrez fur vine alfiette en Rofe, les monrant les vnes fur les autres en pointe de Clocher, puisle Syrop cftant vin peu refroidy, rous le verferez par-deffus, afin qu'il arroufe voltre fruit de tous coftez.

Quant aux Pommes, il s'enconfit fort peu au Sucre, fi cen'eft en Compolte, (c'eft à dire pour eftre mangées chaudes) mais pour garder, l'on $n^{\prime}$ en fait guere, à caufe quelles font mollaffes, \& n'ont pas grand gouft.

Pour les Compontes, celles de Calvil feront confites fans peler \& entieres, leur oftant feulement leitrognon le plus proprement que l'on pourra, en le creufant par l'endroit de la queuë auee vn cô̂teau fort eftroit comme ceux d'Angleterre; puis feront parbouillies, \& bien efgouttés auant que d'eftre mifes dans le 
poefín : A vne liure de fruit, il faut at moins fix onces de Sucre, pour faire vn beau Syrop en geléc ; \& au lieu d'eau, vous ferez bouillir des pelures de Pommes de Renette ou Cappendu : \& les ayant paffées \&z preflées à traulers vne Eftamine ou gros Linge: vous prendrez de ce jus, \& en metrrez vne fuffifante quantité pour confire voftre fruit, le moins que vous pourrez pourtant, de crainte qu'il ne fe defpece: vous le Ferez cuire à grand feu de Charbon (celuy de bois eftant du tout incommode als Sucre) afin que le boüillon furnageant le fruit il le confife également: vous remuërcz fouluent voftre fruit, l'écumant \& tournant auec yne petite gafche de bois, qu'aurez faite de quelque rond de boëtte à Confitures: c'elt vne petite vftancile fort propre à manier le Sucre, tant en Confitures, au liquide qu'au fec, Paftes, Conferites, \& Malfepain's, d'autant que l'on efcume, tourne \& mefle rout ce qui eft dans le poeflon jufques aus fonds: le graueur en a fait la figure tout au bas de la planche, qui eft au commencement de ce Traitté.

Voftre fruit eftant confit, vous tirerez le Poeflon du feu, \& le lailferez prefque refroidir auant que d'en ofter vos $\mathrm{Pom}_{3}$ : 
$222^{\circ}$ LE IA R D I N IE R

mes, les roulant doucement dans leur fyrop auecla gafche, puis vous lestirerez \& poferezfur leur troli, afin qu'elles s'égouttent: en aprés vous les drefrerez en pyramyde furvne affiette, vne au milieu, fix autour, troisdeffus, \& vne fur les trois quiferala poinde: vous verferez vos égouttures dansle pocflon, \& ferez cuire voltre Syrop fur vin petit feu, jufques a ce qu'il foit en gelée, le remuant foutent, de crainte qu'il ne brîle: puis quand vous voudrez feruir vothe fruit, vous le répandrez auec vne cueillir d'argent fur vos Pommes, les arroufant de tous coltez, celafera vin cottignac ou gelée qui ornera de beaucoup voftre fruit; remplifiant aufi le fonds de l'afliette.

Les Pommes de Capendu, \& Renette fe polent, \& fe confifent entieres, ou par moiciées, leur oftant auffi les trognons, \& leur faifant vne gelée auec leurs peaux, comme j'ay dit cy-deffus.

Aux vnes \& aux autres, fi vous y voulez adjoufter le Vin, vous mettrez le rouge au Caluil, auec la Canelle \& le Girofle, \& le blanc aux autres auec le Fenoiiil, cela augmentera de beaucoup leur gouft.

Auec de la Pomme, l'on contrefait des Brunes qui eft en les coup pant par quar. 


\section{Fr a i ço is.}

tiers, \& de chaque quartier en tailler vn petit plotton, l'arrondilfant en pointe par les deux bouts, de la melme forme d'v re Prune ; puis les faire parboüillir, \& de leurs peleures en tirer le jus pour époiffir la gelée, en les confifant comme cy-deuant : eftans cuittes on les dreffera furl'Afliette, en forme de pointe de clocher ou Pyramide: c'eft vn plat fort agreable à voir, \& qui furprend à l'abord ceux qui n'en ont point encores veu, ne pous uant dire ce que ce peut eftre.

Vous pourrez faire de la gelée de Pom. mes à part, en tirant du jus des peaux $\&$ trognons, dont vous aurez ofté les pepins auant que de les mettre cuire : le tout eftant bicn cuit en l'eau, palfé à l'E. ftamine, \&z biea preffé, vous mettrez dans ce jus du Sucre en fuffifante quantité: ce que l'experience vous apprendra, en y mettant plutoft plus que moins, faifant cuire cette gelée en bonne confiftence, la remuant incelfamment, puis vous la paffere à trauers quelque morceat de linge de quintin, la coulant dans des boettes ( pour garder en forme de Cottignac) ou fur des afiettes pour la feruir fur la table: defquelles affiettes fi vous voulez vous en prendrez quelques-vnes, \& couperez ce Cotrignacen petites ban: 


\section{LE IAR INIER}

des, pour orner les bords de vos plats de Compoftes, ou pour ( tirant à l'épargne \& ménage) en feruir peu, les entrelacant en treillis ou figure de ferpenteaux fur des affiettes.

Pour cequi eft des Paftes, je n'en parleray qu'à la Section fuiuante quand je craitteray des Confitures feiches, dautant qu'elles fe font toutes prefque de la mefme façon.

Les Prunes fe conffent pelées \& fans peler en leur laiffant les noyaux, \& l'on ratiffera feulement les quenës pour les rendre plus vertes : on les jettera dans I'eau à mefure que l'on les pelera : elles fe veulent cueillir pour confire, \& non pas ramalfer celles qui tombent le vray temps pour les cueiliir, eft quand celles qui prennent couleur commencent à rougir : les plus cxellentes que l'on choifit, font le Perdrigon, IImperiale, la Diaprée, l'Abricot, la Brignolle, l'Ine vert, Sainte-Catherine, de Roy, \&zau. tres qui fe trouuent trés-bonnes: celles que l'on pele ordinairement, ce font celles de quila peau deuient rovge en meuriffant, laiffant les autres fans peler, fi ce n'eft pour plus grande delicateffe; car pour ce qui eft du gouft, la peau de touces fortes de Fruit eft la partie la plus fa- 
noureufe de tout le fruit : l'on les fera parboiillir ou éuerdumer dans peu d'eau; \& égouter comme nous auons dit cydeuant : puis ayant mis aे chaque liure de Fruit cinq quartrons de bon Sucre calfé auec vn peu d'eau de celle dans laquelle clles auront efté parboiillies, l'on jettera le tout dans la poenle, \& la mettra-t'on fur le fourneau, où le Charbon ne fera qu'à demy allumé, a fin qu'en s'acheuant dembrazer, le Sucre ait loifir de fondre, \& que la poefle ne brûle point.

Vous remarquerez cecy, qu'à toutes Confitures au Sucre; le Charbon ne doit eftre qu'à demy allumé, quand l'on commence à confire, pour les caufes que je viensdedire, \& s'il fe peut, il faut tafcher à lesfaire toutes d'vne cuitte; c'eft à dire, fans les tirer de deffus le feu, que vous ne croyez qu' elles foient faites; car de les tant remettre de fois fur le feu, cela diminuë beaucoup de leur couleur, \& fait brûler le Sirop, qui s'attache au haut de voltre poefle ou bafine: Il fuffic que vous vous y teniezattentif, écumant foigneufement, \& retournant ou faifant rouler le fruit dans fon Syrop, auec le dos de l'écumuire ou la gafche, le faiSamt toújours boüillir par deflus le fruit: 
226 LE IARDINIER

\& lors que vous verrez que le boüillon - s'allentira, c’eft vn figne qu'il approche de fa cuirron : ce que vous reconnoiftrez encore par les gouttes que metzrez fur vine aflette, \& qui ne couleront point.

Vos Confitures eftans faites, vous les tirerez du feu, \& les gounernerez de la mefme façon que j'ay dit aux Poires.

$\mathrm{Si}$ vous voulez en dreffer dans des taffes, quand elles feront fufifamment cuittcs, \&z prefque refroidies, vous les égoutterez de leur Syrop, \& les rangerez proprement dans les talfes, fans les prefler par trop: puis au bout de decix bir trois jours, vous les égoutcrez er. core sily a quelque humidité, \& ferez rechauffer le Syrop, ( $s^{2}$ il eft trop clair vous le recuirez) puis le coulcrez à trauers le quintin fur vos Prans, fans les détacher de la tafle, \& le Syrop turnageant par deflis, les garantira de chancir: vous les lailferez découtuertes trois jours, \& aprís vous leur mettrez vin rond de Papier de la grandeur de la tafie, qui touchera au Syrop, afin que s'il chancilfoit, l'on en changeaft: cela fait que les Confitures paroillent beaucoup, \& fans comparaifon plus belles quand 01 2. Leué ce Papier: qui auec foy entraira: 


\section{FRA N çOIs.}

toute la chancilfeure ou candiffeure, qui d'ordinaire fe fait en forme de peau fur toutes les Confitures, aufquelles on me met point ce Papier.

Pour les Compoftes de Prunes que $1^{\circ} 017$ veut manger chaudes, vous ne metrea que demie liure de Sucre à la liure de fruit: \& pour la proprieté l'on leur coupe la moícié de la queué, quirctte pous les prendre amec les doigts, fil'on ne fe veut ieruir de fourchettes.

Les Amandes \& Abricots vert, font les premiers fruits qui fe confifent : on les prend tendres, auparamant que le bois du noyau commence a le durcir: Pour le confire, ils s'éuerdument dans l'eau claire, y mettant vn peu de bou tarre pour détacher la bourre qui eft delius : puis apres on les effuie chacun à part, pour ofter, cetce bourre, \& on les confit commej'ay monftrécj-deuant, metant feulement liure à liure de Sncre $\&$ de fruit : 11 e eft pour manger en Compoite, il fuffira de denic liure de Sucre: la liure de fruit.

Les Abricots eftans en leur parfaite greîleur, fe conílfent peloz \& fans peler : on leur pouthe le ayau eomme iay ditcy-derane :ux Abricots iechez: alix plus veres, on liur doane vin pecic bouilE $\times j$ 
228 IEIARDINIER

Ion pour les éuerdumer, puis fans lex fecher font pris auec l'écumoire, \& $\mathrm{mis}$ lans le Sucre callé, auec vin pell d'eau; en apres font confits \& gouuernez jufques. à la fin de la mefme façon que les Prunes: Il faut cinq quarterons de Sucre aे la liure de fruit.

Rant à ceux qui font trop meurs. pelez \& non pelez, il les faut mettre parmy le Sucre calfé, auec fort peu d'eau, fans les faire parboillir auparauant ; \& il ne faut craindre qu'ils fe defpecent, car 12 force du Sucreles faifit, \&z on les. retire de la poefle plus entiers ( $s$ sil faut ainfi dire) que l'on ne les y a mis.

Aucuns y mettent les Amandes de leurs noyaux, en plaçant vine à chaque vuide d'entre les Abricots qui font dans les talfes: fi vous y en voulez mettre, 1e vous confeille de les faire confire à part, dans vn peu de Sucre; car fi vous les mettiez fans cuire, ils feroient décuire voltre Confiture, \& elle chanciroit.

Quelques-vns auffi confifent les Abricots d'vine autre maniere, qui eft de les peler, \& au lieude les mettre dans l'eau, ont du Sucre en poudre qu'ils jettent par deffus: les laiffant infufer vn jour ou deux, tant que le Sucre foit fondu; puis ils les mettent fur le feu, \& les ayant 


\section{FRANçoI S.}

retirez apres le premier bouillon, les laiffent repofer encore deux autres jours dans leur Syrop; an bout defquels ils les achenent de cuire, \& mettent les Abricots dans les Pots puis ils font recuire le Syrop, \& le verfent par deffus : cette façon de confire eft embarraffance, \& ne fait pas fi bien que celles que ie vous ay dit $c y$-deuant.

L'on fait auffi de tres - bonne Marmela. ded Abricots, Pefiches \& autres Fruicts propres à ce fujet, en les prenant bien meurs, \& les faifant cuire auec le Sucre, y mettant la moitié de demy- Reptier d'Eas à deux liures de Sucre, \& trois liures de Fruict : vous la cuirez en confiftence four garder, puis la mettrez dans les Pots\& Talfes en les couturant \& gouuernant comme les autres Confitures.

Il faut que ie falfe ic: vne petite digreffion touchant le Sücre \& que ic dife, qu'il $y$ a vin vieil erreur qui s'eft toufiours pratiqué, parmy beaucoup de ceux qui $\mathrm{fe}$ mentent de faire des Confitures : C'eft qu'ils cuifent leur Sucre à part, jufques en confiftence de parfait Syrop, leefcu. mant foigneufement, puis ils mettent leur Fruict dedans \& le confifent : quant à moy, l'experience m'a fait connoiftre qu'ils fe trompent tres-lourdement, pour 
230 LF I A R D I N I E K

les raifons que ie vous va is declarer, qui font, que le Sucre eftant en Syrop, exhale (auec l'Eau qu'ils y ont mis) la meilleure, \& plus fubtile qualité du Sucre, qui penetre \& faifit plus viuement le Fruict : ce qu'au contraire, celuy quieft defia en $\mathrm{Sy}$ rop, ne peut confire que le deflus, à caufe gu'il eft engraiffé, \&z efpoilly par la cuiffon: outre cette raifon, iama is le Syrop ne prend que bien peu le gouft du Fruict, ce quile rend moins agreable.

Les Alberges \& Pefches, comme elles font affez femblablesaux Abricots, anfir veulent-elles eftre confites de la merme façon, en tout \& par tout. C'elt pourqutioy ie n'en diray ricn dauantage; de crainte que par vne fuperfuiré de difcours ie ne srofille partrop nofre Liure.

Les Coings eftans des Fruicts tres-durs, veulent eftre confits dautre façon que les tendres: Pour y paracnir, ils ferone pelez promptement, coupez par qualtiers, \& les Trognons eftans oftez, feront icttez dans l'Eau claire: Par apres ils feront mis boüillir, iufques à ce gu'ils approchent d'eftre cuits puis on vuidera l'Eau dans laquelle ils auront bouilly, \& l'on y mettra de bon vin blane, continuent à les faire cuire, tant que vous voyez qu'ils fe weulonedifpecer,alors vous les tirercz dis. 
FR A \& C O I s.
feu, \& les mettrez elgouter \& feicher: C: la fait vous les confirez comme les autres fruits precedens, trois quarterons de fucre eftans fuffifans pour chaque liure de fruit. $y$ adiouftans la Canelle \& le Cloud de Giroffle \& les metrez dansles pois au fortir de la Poëlle, fansautre ob. feruation que de les pofer en lieu fec.

Le Cotignac à la mode d'Orleans, fe faic en mettant bouillir en eau claire les peaux des Coings, \& les Trognons, y nellan audi la gomme que tirerez des. pepins, qualurez faie tremper, laquelle palforczàtrauers vinlinge, pour lesen Ceparer, auant que de la mettre parmg les peaux : le tout ayant bicn bouilly, il 1e faudra paffer par l'Eftamine, puis faire cuire le ius tant qu'il efpoiffife, $y$ adiouftant du vin blanc François, ou Bourguignon, d'autant qu'il yades vins qui noircifent en cuifant, comme font tous les vins d'Orlcans, de Blois, \& quelques autres : vous y mettrez du fucre à proportion du ius que vous aurez, ne rousen pautant dire la dcze, parce que ce fera plus ou moins, felon gue voltre jus fera fort ou foible, l'experinnevous. l. fera connoifte dés le promier coup: cuis fait, vous le mettrez cuire à feu. mediucre, en l'efcumant bien, \& le se. 
232 LEIARDENAE

muant foutent auec la gafche, pour l' em pefcher de s'attacher à la Poëlfe: \& quand vous reconnoiftrez qu'il fera cuit en confiftence de Gelée forte, vousle tirerez du feu, le verfant à trauers quelque linge dlié, fur les mouftes de plomb figurez, ou tout d'vn coup dans les Boërtes où vous le voudrez conferuer.

Eftant bien froid, pour retirer celuy des: moufles, \& le mettre dans des boëttes, faudra faire chauffer de l'cau dans que!que chaudron, tremper le moufle dedans iufques au bord, puis le coëffant auec le fonds de la boette, retourner les deux enfemble s'en deffus deffous : le Cotignac quittera le plomb, \& tombera dansla bo ̈rte, laquelle sous efgouterez de l'eau qui pourroit y eftre auec le Corrignac, qui fera fondu par l'eat chaude, en fe deftachant du Moufle: ous les lalferezdenx iours fans coururir, aubout defquels vous les fermerez, \& ferrerez én lieu fec.

Si pendant l'anne ce Cottignac chan. cilfoit, ou candiffoit, ou bien quil $y$ tombaft quelque ordure, ilfaudroit faire chauffer de l'eau claire, \& en verfer par deffus; ce la emportera toute l'ordure, \& il fera auffi beau que s'il venoit d'eftre fait. 
F i a çoIs.

Les noix font les confintures les plus loisgues à faire de toures, on les choilira tendres, auparauant que le bois fe falfe à la coquille, \& les pelera. on proprement, leur baillant trois ou quatre coup de couAeau à chacune, en long \& non en traa uers, afin qu'cn bouillant elles foient mieux penetrées : à mefure que l'on les pelera, l'on les iettera dans l eau tiede, \& les fera on infufer en grande Eau fur la cendre chaude, iufques au lendemain, que l'on les mettra bouillir en nouuelle cau, \& eftant tirées du feu \&z efgoutées, feront mifes de rechef infufer dans d'au. tre eau, \& cela vous le rëitererez tant de fois, que reconnoiffiez qu'elles nayent plus d'amertume, apres quoy vous les acheuerez de cuire en bon vin blanc Françoit, à vn cent de Noix, vne pinte de vin, Sx autant d'eau, y mettant gros comme vue Noix feiche de bon Salpeftre pour les nieux penetrer, \& defarmertumer : au deffaut du falpetre, vous y metrez gros comme la moitiéd'vne Noix de Sel; puis quand vouis verrez quiclles feront cuittes. $3 x$ qu'elles fe voudront defpecer, vous les irrerez horsda l'eau, \& les mettrezbien efgouter \& feicher dans queląue linge: ce qu'ertant fait vous les larderez de bonne efcorce de Cirron. \& les metirez 
234 LEIADINIER

confire comme les autres que i'ay dites cy-deuant, $y$ adiouftant vn peu d'eau pour faire fondre le fucre, \& les affaifonnant de Canelle, \& de Cloudde Giroffles : il fuffira de trois bons quarterons de fucre pour liure de Noix, que peferezà l'heure que les voudrez mettre dans le fucre: eitans confites \& le Syrop reduit en bonne confiftance, vous fes verferez dans les pots, les lailfant defcomuerts quelques jours comme les autres.

Les Cerifes que l'on voudra confire, feront choifies les pius grofles, mieur nourries, \& non tournez : on ne les prendra trop meures, mais quand elles allront vne couleur viue \& vermeille par tout ; celles de la Vallée de Montmorency font fort en eftime pour leurbonté : c'eft pourquoy l'on prendra fon temps, quand on commence à les apporter à Paris, \&z on $n^{3}$ attendra pas la fin de la faifon, où l'on n'en peut auoir que de lieu ombragé : car vous. fraurez, qu'entoutes fortes de fruis, les premiers qui fe mangent en chaque faifon pourueu que l'on leur donne le temps de meurir) font toûjours les plus excellens au gouf, \& les mieux conditionnez: les autres n'efans que comme des regains, qui par debilité n'ont peu aecompagner les premiers en maturité. 
Pour confire vos Ceriles fans noyau, il faut les pefer atuant que d'en tirer les noyaux : puis auec le plus de proprieté que vous pourrez, tirer le noyau en pref. fant doucement la Cerife du bout des doigrs, \& tirant laqucuëde l'autre main il fortira aifément fans la defchirer: Vous les ietterez à mefure dans voltre Poëlle où il yaura du fucre en poudre, que vous aurez auli pelé auant que de $1^{3}$ y metre : vous verrez par le poids, ce qu'il y aura à defduire pour les queués \& noyaux, afin de garder la doze neceifare du fucrequ'il conuicnt metrre àchaque liure; qui eft, cinq quarerons de fucre pour chacune liure de Cerifes, I vous les voulez faire belles à prefenter, \& qu'il y ait beancoup de Syrop : Ie fçay bien quall feroit plus expeditif, apres leur auoir ofté les noyaux, de les pefer dans quelque papier. mais cela les gafteroit \& patrouilleroit, ceque la Cerife ne peut fouffrir à caufe de fí delicatelle, fe tournan facilement, is auli qu'elle perdroit beaucuup de fon ius : Pour fondre le fucre vous y metrez quelque peu dz ius de Cerifes, qu'aurez prellées dans vn linge, ( les tournées feront altezbon. nes pour cela) Vous metrez la Puëlle, fur le fou, le charbuir n'eltant qu'à demy 
236 I I I A R I N TER

embrafé, pour les caufes que iay dites cy-deuane: vous cuirez vos Ceriles tout d'vn bouillon; puis yous les tirerez dis feu, les laiffant prefque refroidir, \& les. drelfere $z$ dans vos pots \& 2 tafles.

Si vous voulez en dreffer dans des taffes. comme les Prunes, n'en mettant qu'vn. lit, \& les laiffant deux ou trois iours fans y mettre le Syrop, \& le rond de papier qui toucheau Syrop, elles feront belles aे merueilles.

Quelques-vns $y$ adiouftent le ius de Gadelles, mais elles changent le gount de la Cerife par leur acidité, \& rendent le fyrop trop liéen Gelée: Si pourtant vous vouleztirerau mefnage en y en mettant, il ne faudra qu'vne livre de fucre, à vne livre de fruit.

Pour framboifer vos Cerifes, à la place du noyau de chaque Cerife, vous y metrrez vne Framboife rouge, les ayant pefées auparauant, pour éuiter confufior à la dofe du fucre qu'il faudra mettre.

Les Cerifes où l'on n'oftera point le noyau, feront piquées chacune en deux en iroits auec la pointe du coufteau, cela empefche qu' elles ne creuent, \& quittent leur peaurvous les confirea comme delfus, vne liure de fucre eftant fuffifante à rne liure de fruit. 


\section{FR A N ÇOIs.}

Les Griotres eftans vne efpece de Cerifes, feront confites de la mefrme façon que les $C$ erifes: fi vous y voulez mettre le ius dc Gadelles, cela reueillera leur gouft qui eff allez plat: elles fe veulent contire bien meures à caufe de leur fermeté.

Le firop de Cerifes fe fait auec du ius de Cerifes bien meures, que pafferez à trauers vne Eftamine, ou linge fort ; \& y adiouftant le fucre, ( vnelïvre à chaque pinte de ius ) vous le cuirez en confiftence de firop.

Pour le clarifier il faudra le couler à trauers vne fueille de papier brouillars, ou dans la Chauffe d'tipocras.

Pour faire la gelée de Cerifes, il faut paffer des Ceriles à trauers l'eftamine, \& y adioufter vn tiers de ius de Gadelles, puis à chaque pinte, mettre yne liure \& demie de fucre, \& la cuire à petit feu, en tournant toújours de peur qu'elle no fe brufle: \& pour connoiftre quand elle fera faire, vousen mettrez quelque gouttes fur vne aflette: fieftans refroidies, vous les pounez lencr auec la pointe du coufteau, la gelée fera faite, finon il faudra continuer à la cuire : Vous la mettrez dans des boëtes comme le Cottignac.

Il y en a qui font des confitures de $\mathrm{Ce}-$ 
233 LE IARDINIER

rifes lans fucre, en palfant force ius de Cerifes \& de $a^{2}$ delles enfemble, le faifant cuire \& le reduifant iufques au tiers, puis ils en confifent leurs Cerifes : c'eft vne commodité pour ceux qui n'ont point de fucre, mais cen'eft pas $r n$ grand ragount pour les bouches delicates.

Aux Compontes de Cerifes, l'on y lailfe la moitié de la queuë \& le noyau par confequent: ce n'eft d'ordinaire qu'à la noutucauté quel'on en fert fur table il fufira de demie liure de fucre à la liure de Cerifes, d'autant que celt pour manger à l'iftant quielles font faites.

Pour les feruir fur l'affette, l'on dreffe toutes les queuë en en-haut, puis l'on verfe le firop tout chaud par deffus à l'inftant que l'on les veut feruir fur table.

Ie vouls veux enfeigner la maniere de faire l'Eau Clairette, qui eft vne compofition tres-excellente pour rechaufier l'eftomach des Vieillards, qui par mangue de chaleur naturelle, ont fouvent des cruditez \& indigeftions; elle eft aufii tres bonne pour les perlonnes de toutes aagesqui ont des debilitez de cœur, \& des efpeces de coliques, caufées par les brouillards ou froidures, cette eau les confortant extremement, \& les remet- 
tant en parfaite fancé: les Femmes en tra. uail d'enfant en peuuent auffi ver, pour leur augmente: les forces $\&$ le courage.

Pour la faire, vous prendrez deus pinres de bonne eau de vie, fi elle eft rectifiée elle en fera encore plus excellente : vne liure des plusbelles \& plus grolfes Cerifes, aufquelles vous ofterez feulement la queü̈: vne liure de fucre, demie once de Canelle, \& demie once de Clou de Giroffle: Vous mettrez le tout dans vne bouceille de verre, infufer au Soleil iufques à la fin des iours Caniculaires, remuant parfois cette compolition auec vn bafton, ou bieis renuerfant laboureille, tour le marc qui eft au fonds fe meflera par tout, la tenani toûjours bien bou. chée crainte de l'éuent.

Plein vne cuillier d'argent fuffira pour vne prile; $8 z$ vne Cerife que vous tirersz de la bouteille auec vn fild'Archail, fait en petit darel, afin qu'il apporce la Cerife qu’aurez piquée.

En temps de pefte, c'eft vn tres-bon \& agreable preferuacif: vous en prendrez, \& en donnerezà vos domeftiques vne prife à chacun à ieun, \& ne faudra manger de demis heure apres, afia que les elprits qui font dans cette compofition fe refpandent par tout le corps. 


\section{IE IARD:NIER}

Les Grozeilles blanches ne fe confirent guere au liquide, fi ce n'eft à la grande nouueauté que lon en fait des Compoftes pour feruirchaudes furla table: elles fe confifent ordinairement au fec, nous en parlerons au chapitre fuirant.

Pour les Compoltes, l'on coupe la teite \& la queuëdes Grozeilles, \& on les fait parbouillir; puis eftans efgouttées on les pefe, Ei on les corfic tout d'vn boüil lon; demie liure de fucre à la liure de fruit fera plusque fuffifante pour les rendre agreables.

Les Grozeilles rouges \& rerlés que nous appellons Gadelles, fe confifent au liquide, eft vn petit fruit extremement agreable.

Pour les confire vous les peferez, 8 les fcparerez de leurs qucuës, les metrant dans la poëlle ou fera le fucre en poudre: par apres vous y adioufterea du ius d'autres cadelles quaurez preffées doucement dans l'ếtamine, puis les metrez fur le feu, \& les confrez tout d'vn boinl lon:apres quoy vouslestirerez dufeu, les laiflerezvn peurefroidir, \& les reflerez dans vos pots \& talies: Il fuffra d'une liv ure de fucre à vo liure de fruit pour les rendre tics belles: vous y mettrez moins de fucre fi vous voulcz, \& feront bonnes 
à manger : mais non fi belles, ny auec tant de firop.

$\mathrm{L}_{\mathrm{a}}$ Gelée fe fait en preffant les cadel. les, \& tirant par force iulques à la derniere goutte de ius, dautant que le plus efpois ne fort que le dernier, puis vous y adioufterez le fucre, trois quarterons la liure de ius (quieft vne pinte ] eftans fufffans pour faire de telle celée: vous la cuirezen confiftence ( comme iay dif cy-deuant aux Cerifes puis la drefferez dans des boëtes, la lailfant refroidir deux ou trois iours auant de les counrir.

L'on framboife auffi des Gadellesen mettant du ius de Framboifes pour fon:dre le lucre, en lieu de celuy de $\mathrm{G}_{2}$ delles; cela leur donne yn parfum tresagreable au gouft.

Quelques-vns confient des Gadelles fans Sucre, ent tirant \& exprimant quan. tité de ius, le faifant reduire au tiers: puis y mettant conlire les Gadelles, ils les cuifent iufques à ce qu' ils croient qu'elles le foient fuffifamment pour eftre gardées: s'il y adiouftoient feulement vn quarteron de Sucreà la liure du fruit, elles auroient quelque petit agréement quiles foroit manger, mais lans fucre, c'eft voe eftrange confiture.

Lis Iramboifes fe veulent confire \&e 


\section{LE I A R D I N IER}

garder de la mefme façon que les Cerifes, mettant cinqquarterons de fucre à la livre de Framboifes, a nque le Syrop en foit plus beau.

Le Vcrius que vous prendrez pour confire, eft ce uy que l'on appelle Bicanne ou Bourdela, \& duquelà Paris on fait le verius \& que l'on pile aux Preffoirs de Sainte Oportune, du pont Saint Michel, \& autresendroits, lequel par fa grolfeur \& efpoiffeur de chair, fait la plus belle \& agreable confiture.

Vous le choifircz non ttop mollaffe ou meur, mais dans fa beauté, auant que le goult s'adoucilfe; vous le peferez éfrené de fa raffle, \& le fendrez par la moitié en lonz, oftant tous les pepins s'il fe peut, en le iettant dans l'eau claire, à mefure que le fendrez; puis vous le ferez parbouillir, mettant vne petite pincée ou deux pincées de Sal peftre, pour Iuy conferuer fa couleur verte : Eftant a morty feulement, vous le tirerez du feu, $\&$ le courirez de quelque linge, le lairfant refroidir dans fon eau, l'efgouterez \& le mettrez dansle Sucre en poudre, $y$ adiouftant vn peu d'autre Eau claire, pour fondrele fucre feulemeni, puis vous le confirez tout d'vne cuitte, carfi de tiriez du feu pour l'y remettre, il per- 


\section{F R A çOIs}

droit beaucoup de la couleur: Eftant cuit en bonne conliftance, yous le retirercz du feu, le verferez dans quelque terrine, pour ne le laiffer dans la poëile chau. de, qui deftruiroit auffi beaucoup de $f_{a}$ conleur; \& epres le dreflerez dans vos pots \& taffes, ainfi que les Ceriles: Il faut liure à liure de fucre \& de fruit, pour rendre voltre Verius bien beau.

Il s'en confit aufin fans fucre, ou auec bicn peu : mais c'eft vne confiture fort de-. fagreable, quidégoufte pluftolt les pauures Malades à qui l'on les donne, que de leur refuciller l'appetic.

$\because$ vous voulez, vous ferez de la gelée de Verius, de la mefme façon que iay dit aux Grozcilles, y adiouftant du ius de peaux de Pommes de Renetre ou Capendu, pourluy donner quelque liaifon, ou bien de la Gomme de pepins de Coins, elle fera fort agreable à manger à caufe de fon acidité.

Les raifins Mufcats feront fendus comle Verjus pour leur ofter les pepins, St en melme temps iettez dans la Poëlle fur le fucre en poudre : l'on en preflera quelques grappes pour en tirer du Mouft, afin de donner vne fuffifante humidité au Sucre pour le fondre: car fi rous en donnicz trop peu, le fucre ne fondroit 
244 LE IA R D I N I B R

fondroit pas tout, \& les plus gros morceaux fe maintiendroicnt en forme de Conferue: C'eft pourquny en ce fruit-cy \&r en tous autres, vous y mestrez du defcuit, (c'eft à dire eau ou ius, ) vne fuffifante quantité : ce que $l^{\circ}$ experience vous apprendra en peu de temps: Car aufin d'y en mettre par excez, il faudroit que vos Confitures fuffent plus long-tęmps fur le feu pour exhaler le trop d'humidité qu'il y auroit, \& cela les defpeceroit par trop de cuilfon, \& gafteroit la couleur.

Pour les rendre beaux, il faut cinq quarterons de fucre a la liure de mufcats, afin qu'il y ait quantité de frop, qui eft la grande beauté en ces confitures-là : Vous les confirez, puis les dreflerez dans des talfes, n'y metrant qu'vn lit de Mufcat : \& au bout de deux iours vous verferez le firop deffus, \& les coutrirez \& gouuernerez comme les Cerifes.

Les autres Raifins feront confits fans autre façon que de les faire vn peu amortir au Soleil ou au four, \& les efgrener fans les fendre : mettre liure à liure de fucre $\&$ de fruit, puis les cuire en bonne confiftencé, \& les dreffer dans des pots \& talles les goumernant comme des Mufcats.

Les. Carottes \& Paftenades $f$ confifent 
au fincre, \& fe feruent ordinairement chaudes en compofte: Pour les confire, vous choifirez les plus belles \& plus nettes de nouds, \& racines: vous les ratiferèzles iettant dans l'eau tiede, puis les ferez parbouillir, iufques à ce qu'elles attendriffent ou amollifient; par apres vous les tirerez du feu, vous les efgouterez \& feicherezdans vn linge : cela fait vous.les mettrez dans le fucre en poudre atec vn peud eau, \& les ferez bouillir tout d'vn bouillon, \& les trancherez en long ou en roëlles, puis les ayant dreffées fur des affiettes, vous iettcrez le firop par defífus. Ie trouue vne autre methode de les confre beaucoup plas facile qui eft de les mettre dans vil pot de terre comme des poires de Carreau, lis faifant cuire premierement auec leau, puis aux rouges y adioufter le vin rouge, auec le fucre, la Canelle, \& le Cloud de Giroffle : \& aux autres qui font iamnes \& blanches, le vin blanc François, aulec le fenouiil vert, le firop eftant de bonne confiftence, vousles taillerez, drciferez fur des Afliettes, \& ietterez le firop chaud par deffus : il n'ent de befoin de mettre plus de fix onces de fucre à chaque liure de racines.

Les Neffles fe cuifent au vin dans la L iij 
246 LE I A R EIXIER

poëfle à fricalfer, leur ayant ofté les aifles \& la queuë; puis l'on y met du fucre, \& quand le firop eft prefque fait; on les dreffe \& les poudre-on de fucre pardeffus

Le firop de Limons fe fait ou par infufion, ou par cuiffon: Par infufion, en mettant à vne liure de ius de Citron deux liures de fucre en poudre, \& l'expoler au grand Soleil: Pas cuiffon, en mettant à vne liure de ius, vne liare \& demie de fucre calfé, \& le faire cuire vn peu moins que iufques à la confiftance de firop; qui eft qu'enen mettant fur vne Affiette, il necoule point puis vousle ferrerez dans des boutejlles de verre fans Ozier, afin que sil vouloit chancir, on le mift au Soleil, ou dans le four, apres que l'on auroit retirée la fourné de pa in : Si vousy voulezadioufter le Mufc \& l’Ambregris, vous la rendrez femblable à l'Aigre de Cetre.

La Limonade fe fait auec le dedans des Citrons, oftant les plus grolfes peaux qui feparent les graines \& les mettans tremper en eau claire, puis wous y adioufterez du fucre en poudre, la quantité que iugerez eftre neceifaire \& mettrcz auffi tremper dedans quelque peu de Coriandre, cafféc $\&$ enfermée dans vn potir linge, auec quelque morceau de Canelle: 


\section{Fra nço'is.}

Le wufc \& l'Ambre y pourront eftre adjouftez pour l'affaifonner plus delicieufement.

Vous laifferez infufer le tout enuiron demy iour, le verfant fouuent d'vn vaiffeau en vn autre, \& preffant auec la main les Citrons qui font dedans: Vous y goufterez pour inger ce qui y manqucroit à la rendre excellente, \& apres l'auoir paffée à trauters vn linge fans preffer le Marc, vous la mettre 2 dans des bouteilles pour eflre feruie aux Dames : Cette boiffon ne dure pas plus de trois iours en fa bonté, c'eft pourguoy l'on ne la fera qua au befoin.

Il y en a qui y adioufte les amandes pilés : mais commec'eft vne boilfon, la plus fimple qu'elle pent eftre eft la meilleurc. Les meures fe doinent prendre à dem noires pour eftre en leur bonté : elles fe confifent en mettant trois quarterons, de fucre, à la liure de fruit, \& feront cuir tes toutes d'vn boiillon comme les Cerifes, puis ferrerez dans les pots: l'on en fait auffidu lirop à part pour le mal de gorge, que vous façonnerez comme ccluy des Cerifes. 
348 LEIARDINIER

Des Confinies an Sec, Paffes de friats.

$$
\text { SECTION VI. }
$$

Es Confitures Seiches font eftimaL. bles, en ce qu'elles s'enuoyent de loing, \& fe prefentent aux perfonnes des plus hautes qualitez, quiles eftiment cant pour leurbonté, que pour la commodité qu'ils ont de les porter en la pochette, à caufe de leur friand gouft, \& pour leur faire bonne bouche au fortir du repas.

Il fatit en vous donnant la methode de les feicher, allerd'ordre cnmme i'ay fait aux autres fections. Ie commenceray. donc par les Poires, \& vous diray que les ayant confites (come i'ay dit cy deuant) \& eftans preftes à tirer du feu . fi vous voulez les mettre au fec dans le mefme iour, vous leur donnerez quelques hoiillons dauantage que pour garder au liquide; \& ce iufques à ce que le Syrop fe vueille congeler en Conferue: alors vous tirerez voltre poëlle du feu \& mettrez vos poires fur des Ardoifes ou fueilles de ferblanc, pour les porter feicher dans l'eftuue.

Cette Efture eft vn petit cabinet bien 


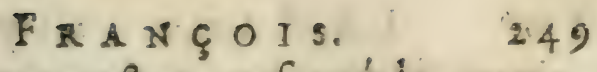
ferméde tous coltez, referué la porre, par laquelle vous palferez pour ranges vos Ardoifes, fur les tablettes defquelles il. fera tout entouré, \& mettrez à bas vne poéle, ou rechaut de feu, pour deflecher le tropd'humidité, qui refte en vos confitures, les tetournant, \& changeant de place, autant de fois que vous iugerez qu'elles en auront de befoin.

Si vous ne voulez feicher vos poires qu'a mefure que vous en aurez affaire, ( à caufe quelles ont de beaucoup plus belles fraiches faites que vieilles feichées:) vous les garderez au liquide, \& quand vous les voudrez fecher, vous ferez fondre quelque peu de fucre, comme vin quarteron ou enuiron, à chaque liure de poires, \& le mettrez cuire en confiltence de Conferue, (cóme ie vous enfeigneray a connoiftre cette cuilfon en la lection fuiuante: ] puis vous verferez vos confiturcs dedans, leurbaillerez le boüillon iufques à ce qu'elles foient reuenuës à la cuiffon de Conf rue : les tirerez du feu, \& drefferez fur les Ardoiles, pour eftre portées à l'eftuue fecher commedetlus.

$V$ ius les lailferez refroidir enticrement, at:ant que de les mettre danslest boërtes pourlis ferrer, mettant à chanue lie defruit, vne fueille de papier fectié,

$$
\text { Iy. }
$$




\section{LE IARDINIER}

$\therefore$ les rangerez proprement fans les pref́fer, à caufe, qu'en les froilant vous rompriez la glace qui eft fur le fruit: ce quile rendroit blanc de fucre ou farineux, \& enlaidiroit fon luftre.

L'on a de couftume de lier les petites Mufcadilles par bouquets en fortant de l'eltuue, auant que de les mettre dans lespoëtes, feptà chacun bouquet, d'où vient le nom quel'on leur donne de lept engueule, ou bien quele Trocher oû elles viennent à l'Arbre, en produit ordinairement fept, qui àcaufe de leur petitelfe, peuuent eftre mangées d'vine leus. le bouchée : les autres gros Mufcats, Blanguets, petiis Roulfelets, \& autres petites poires femblables, feront liées par trois ou quatre enfemble felon leurgroffeur.

Pourfaire la palte, yous prendrez des poires de meilleur gount que vous pourrez, \& celle qui auront la pea:z de belle couleur. Vous neles pelcrez point; mais ayant ofté la tefte, le trognon, \& la queué; vous les ferez parbouillir, les tirerez du poeflon, les efgouterez, \& lecherez, puis les palferez à trauers l'efta mine, cu au fas, (comme l'on munde la Calfe) par apres vous prendrez les partis gui nauront peu paffer, les pile- 


\section{FRA ÇOIs.}

rez dans le mortier d'Albaftre, \& les pa!ferez auffi: Cela fait; vous peferez yoftre pafte fur quelque papier, la mettrez dans le poëllon auec le fucre en poudre bien delié à proportion : cuirés cette paftc à petit feu, la remuant continuellement de crainte qu'elle ne brufle, s'attachant au poëllon, \& la retournerez incellamment, iufques à ce que vous reconnoiffiés que la pafte quite le poeilon; alors vous la tirerés du feu, \& la drefferésen maccaron fur vos Ardoifes, la mettant feicher dans voftre eftuue; Vous les retournerés\& changerés de place tant qu'elles foient feichées, puisles tireres de l'efture \& les rangerés dans les boëres comme les poires : demie liure de fucre fuffit à chaque liure de fruit : fi vous voulés prendreles belles peaus des fruits que pelerés pour apres les auoir vn peu éuerdumées les piler \& paffer, vous augmenterés de beaucoup le gouft de vos pan es: dautant que le gout le plus relcué du fruit eft toûjoursen la peau.

Quant anx Pommes, la Confiture n'en eft trop exquife, cent pourquoy l'on en confit fort peu : fi vous en voulés confire, vous vous gouternerés comme aux Poires. L'on fait des paftes de Pommes, de[gueclles l'on contrefait los Abricots à 
252 Z E IARDINIER

Oreilles, autant qu'ils font rares: Pour ce faire, l'on prend des Pommes de Renetre, ou de Capendu, ies plus belles quelon peut, on les coupe par moitie. \& on leur ofte la tefte, \& le trognon, puis on les met parbouillir, \& on les paffe comme les Poires: Pour y donner le gouft, on prend des abricots fecs, les vieils d'vn an, \& pallés, y ferone fort propres, \& particulierement ceux qui auront efté confits aucc la peau, ou bien fi vous aués confit des peaur d'A bricots dans la faifon pour ce fuict, ayant o'é le rouge qui y eft, \& ne lailfant que le iaune : ceit au temps que les Pommes ont vin peu paffé leur plus grande verdeu-, qui fera enuiron vn mois apres quelles auront efté mifes dans le fruiticr, que vous en tirerés hors des plus belles, \& des plus faines, potır preparer (comme ie viens de dire) pillerés yos peaux d'Abricots, les palierés au fas, \& lés meflerés auec les Pommes, puis vous peferés le tout, \& mettrés la moitié autant de fucre en poudre que vous aurés de marmelade, par apres vous ferés cuire cette pafte, comme iay dit celle des poires: eftant cuitte, vous la tirerés dufcu, la laiffant vn peu refroidir, \& auec depetits moufles faits en forme d'vn Abris 
cot outuert, vous mouslerés cette palte, \& la ferés fecher dans l'eftuue fur les ardoifes, la retournant à propos: \& quand elle commencera à fe feeher, vous luy donnerés le ply pareil à eeluy des vrais abricots à Oreille, les acheuant de fecher, puis vous les ferrerés dans les boëttes, mettant auffi du papier entre les lits comme aux autres fruits fecs.

Les Prunes feches fe confifent comme au liquide, pelécs \& fans peler, puis on les feche, \& forre dans les boëites, tout de la mefme façon que i’ay dit aux Poires.

Les prunes feches s'enueloppent par fois dans des petits papiers chacune à part pour plus grande commodité, \&e propreté, à les porter dans la pochette. Les Paites de pranes fe font de la inefme maniere que les autres precedentes: on oftera la peau à celles qui font colorées a fin qua elle ne donne aucune teinture à la pafte, mais qu'elle foit d'vne belle couleur verte.

Il fe fait en Bourgongne vne pafte de Prunes de moyeu, laquelle s'apprefte tout de la mérme façon que les autres, referué que l'on ne la cuit pas iufquesà ce qu'elle quitte le poëtlon: mais vn peu moins suittel'on la rerfe dans des buèt. 
254 LE I A R D I N I ER

tes, que l'on lailfe quelque temps refro:dir, fans les couurir, comme quatre ou cinq iours; aubout defquels, on ferme lesboettes, \& on les ferreauec les Cottignacs, pour y regarder de temps à autre, fi elles fe conferuent bien.

Les Amandes \& Abricots verts, ayans efté preparés comme pour le liquide, \& eftans cuits ferout tires de leur firop, \& fechés ainfi que les autres fruits que iay dit cy-deuant.

Les Abricots meurs fe mettent ordinairement à Oreilles; pour les y preparer, il faut les outrir par moitiées siles applatir pour fecher, puis eftans vn peu fecs les refermer, \& leur faire faire à chaque moitié vn quart de tour, en les tirant en long, ils fe rencontreront de la mefme figure que l'on a de couftume de les façonner.

Pour la pafte diabricots, elle fera façonnée comme les precedentes, en faifant vn peu parboiiillir le fruit, \& oftant la peau aux endroits où elle eft rouge, puis les piler \& paffer, [ mettant demie liure de fucre à la liure de fruit) les cuire en confiftence, qui eft iufques à ce que la pafte quitte le pocflon, par apres les drefferparkaccarrons fur las Ardoifes, les fecher \& ferrer comme les auties. 


\section{FRAN çOIS.}

Le Ramage de Genne fe fait auec corpafte d'Abricors; laquelle eftant cuite en confintence, vous la laifferez refroidir, puis ayant poudré vos Mounles auec du fucre delié, paffé au Tamis de foye, lequel vous mettrez dans vin linge alfez fin, \& le fecou rrez fur le Moufle: cette poudre ira par tout, \& empefchera que la pafte ne s'attache au Moufle, ce qu'aya nt fait vous $\in$ mplircz voftre Moufle, \& auec va Rouleau de Paticier appuyerez par tout en roulant fur le Moufle, \& oftant le fuperflus, il reftera la figure du Moinle: cette pafte eftant froidc, vous la renuerferez fur l'Ardoife, pour la mettre fecher aux Eituues, en cas qu'elle foit trop mollatie; ou fi elle a de la liaifon fuffifante, vous la mettrés tout d'vn coup dans la boette, pour la ferrer auec les autres.

Sivous ne voulés faire que des compartimens, ou chiffres, vous mettrés voftre pafte touce froide fur vne table, qu'auréșpoudrée de fucre auparauant, \& l'étendrés aucc le rouleau, de l'efpoiffeur que voulés qu'clle foit : puis auec des modelles de fer blanc, la couper és en le appuyant deffus, oubien vous la taillerés aucc le coufter u en la figure que delirerès : cela fait, vous la me ttrés aus 
296 L I I R D I IER

Eftuues fi elle en abefoin, \& cftant froide le ferrerés dans la boëite comme les autres.

Les Pefches de toutes fortes feront mifesau fec \& en pafte, de la melme manie. re que ie vous ay enfeigné d'accommoder les Abricots.

Pour ce qui eft des Coins, il ne s'en feche que par fantaifie, d'autant que c'eft vn frait qui elt affés dur de luy-mefme, toutesfois fi vous voulésen fecher, vous vous y gouverneres comme aux autres, le faifant pourtant parbouillir, \& $z$ preft à fe dépecer, auant que de le confire, afin qu'il en foit plus delicat.

La pafte fe pourra auti faire comme les precedentes; il n'eft de befoin d'y mettre les peaux, d'autant que d'euxmefmes ils ont \& alfés de goutt, \& allés de liaifon.

Les Noix fe preparent pour eftre miles au fec, en les choififant comme pour le lequide, mais il leur faut ofter tour le ver iufques au blanc; les iettant en l'eau. claire mefure que l'onle pele, puis les defamertumer en les faifant parboüillir endiuerfescaux, comme iay dit aux liquides, \& les confire, fecher \& ferrer, ainf que les autres fruits precedens.

Il ne fe fait point de pate de Noix: 


\section{F R A ço I 5 .}

ceit pourquoy je n'en diray rien.

Quant aux Cerifes \& Agriottes, on les confit pour mettre au fec commeles autres precedens fruits, fe lient par bouquets ainfi que les Mufcadilles, \& on les met à reilles comme les abricots, en les ioignant deux enfomble, qui fe fouderont en fechans à l'eftuue.

Pour la paite, elle fe fait de deux façons: L'une en prelfant des Cerifes bien meures dans l'Eltamine, 8 broyant les peaux dans le Mortier, puis les paffer au Tamis : mettre le tout fur le feu, \& le faire reduire à la moitié:adiouftant demie liure de fucre en poudre, à chaque liure de Suc, \& cuifant le tout en confiftence de pafte : L'aure: que ie trotue beascoup meilleure, eft d'efpreindre vn peus de Cerifes, les froilfant auec les mains, \& ce qui reftera dans l'Eftamine, le paffer all fas, piler les peaux qui pourrront refter, \& les paffer auffi puisy adioufter le fucre en poudre à chaque liure de fruit demie liure de fucre, mettre cuire le tout en confiftence, puis la dreffer en Macarons fur les ardoifes, pour eftre feché aux Eftuues.

Le Irs prouenant de l'expreffion qu'aurez fait de yos Cerilcs, fera confit ess 
258 LE IARDINIER

frop pour garder, \& auffil l'on en pourra mettre dans le fucre pour le fondre, quand vous ferez les confitures liquides.

L s Grofeilles femettent au fec comne les fruits precedents, ayant obferué auparauant ce que i'en ay dit pour les confire au liquide.

Pour les Gadelles, il ne s'en confit point au fec, à caufe de leur peticuffe; mais l'onen fait des paftes excellentes, les froiffant dans l'Eftamine, \& paffant au fasbiendelié, pour en ofter les petits pepins \& la tefte: vous la ferez tout de la mefme maniere que de celle Cerifes.

Les Framboiles Rouges \& blanches ne fe mettent point au fec, non plus que les Gadelles, mais les paftes font be aucoup à eftimer pour leur agreable gouft: elles fe font comme celle de Ceriles y mélant quelque peude Gadelle pour en releuer le gouft parleur petite acidité.

Le Verjus \& mufcats ne fe confifent point auffi au fec: les paftes que l'on en fait font tres-bonnes, \& fe veulent auffi façonner commeles Cerifes, ayant aus parauant preparéle fruit, commeiay dit cy-deuant, quand i'ay enfeigné la maniere de les confire au liquide.

Les Orenges fe confifent enticres ou parquartiers : pour les preparer, il faut 


\section{FRA NÇOIS 259}

raper tout le iaune, auec vne rape de cuiture : puis fa vous les voulez confire entiers, il faudra faire vn trou à l'endroit de la queuë, \& tirer anec patience tout le dedans, lailfant la peau la plus deliée que l'on pourra: on les mettra tremper à melure dans l'cau ticde, par apres on les fera parboiiillir en grande eau pour en ofter toute l'amertume, la changcant plufieurs fois, tant qu'elle n'ait plus aucun gouft d'amer : cela fait vous les tircrez du feu, les icttans dans d'autre eautiede, puis ferés fondre voftre fucre à petit feu; cependant qu'il fondra vous mettrès égoutter vos Orenges, les pofant fur vn linge plufisurs doubles, le trou deffous : apres vous les mettrés dans le fucre, queferés cuire iufques à la confifence de Conferue : pendant qu ils cuiront vous les remurés \& tournerés fouuent auec la gafche, puis les retirerés auec l'écumoire, \& égonterés bien le fucre qui fera dedans; apres quoy vous les mettrés fecher dans l'eftule, ce qui fe fera en demie-heure de temps: au defaut d'eftune vous les mettrez deuant le feu, furde petits brins de balay, ou ils fe fecheront.

Pour celles que vous voudrez mettre parquartiers, ayant rappé tout, le iaune 
260 LE IARDINIER vous couperez la peau en tant de parties que vous voudrez, \& en la tirant proprement, vous la fepareréz du dedans fans rien rompre: \& fivous voulez, vous la fercz beaucoup plus de liée ou tenuë que celles que l'on confit entieres.

L'on coupe auffi de cette peal par petites partie, que l'on nomme des Zeftes, tout de la mefme forme quel'on en prend fur les Orenges, pour en les preffant ietter contre vn verre (où il y a du vin) vn efprit fubtil qui eft dedans, lequel donne gouft au vin àtrauers le verre:ces mefmes Zeftes eftans preffez contre la flammed'vne Chandelle, cét efprit prend feu en petillant.

L'on coupe encore les peaux d’Orenges \&2 (itrons, cn forme de gros lardons lefquels on confit comme i'ay dit cy deuant; puis eftant cuirs, l'on iette dans leur firop d'autres lardons d efcorce de Citron confite bien verte : le fucre eftant cuit iufgues en Conferue, il faut prendre ces lardons anec l'Efcumoire, \& les ayant bien égouttez, vous les drefferez en forme de $R$ ocher, entremeflant les couleurs; ce qu'elant fait vous les feicherez dans l'eftuue, ou les mettrez fimplement de. want le feu.

Fous obferuercz que les Crenges \& 
Citrons, tels que nous les auons à Paris, ne fe veulent confire qu'à mefure que l'on en a de befoin : d'autant qu'ils blanchiffent \& fe farinent quand ils font vieils faits, ce qui eft fort defagreable à yoir.

Pour la doze du Sucre, je ne vous en donneray point, d'autant que le plus quevous y en metirea ne fera pas perdu, feruant à faire vos Conferues \& Maffepains, comme je vous diray en la Section fuiuante, \& aufi quil eft necelfaire que ce fruit foit en plein Sucre, pourfe bien confire.

Vous ne jetterez la rappure de vos peaux d'Orenges, car elle feruira à faire de trésbelles \& bonnes Conferues.

Les Citrons fe confiront tout de la mefme maniere que les Orenges fans nulle referue : L'on taille des tranches fort deliés fur les poncilles, larges de deux ou trois doigts, lefquelles on confit \& feiche, comme celles des Orenges.

Des Laictuès royales fe tire vn Cardon quand elles montent à graine, duquel on fait la Confitures que les Italiens appellent gorge d'Ange.

Pour les confire vous les dépoiiillerez de leur petite peau ou efcorce, les iettant dans l'eau frailche, \& les ferez vn ${ }^{\circ}$. 
262 LE IARDINIER.

éuerdumer; puis les metrez dans le Sircre en poudre auee quelque peu d'eau claire pour fondre le Sucre, apres quoy vous les ferez cuire tout d'vn boiiilion iufques à ce que le Sucre foit en Conferue: alors vous lestirerezauec l'efcumoi- les metrrez feicher fur les Ardoifes dans voltre eftuue, les retournans de tous coltez, \& eftans fecsles ferrerez dans les boettes comme les autres fruits fecs : Si vous voulez à la fin de la cuiffon y adjoufor vn peu d'eau de fleurs d'Orenges, pour leur bailler quelque odeur, à caufe qu'ils font infipides, cela les ameliorera extrémement.

L'on pourra auffi confire beaucoup d'autres fortes de fruits, comme Melons, Concombres, mefme des Citrouilles, fi par curiofité ou par excefliue dépenle, on vouloit faire voir des fruits, confits de toutes fortes.

Des Conferwes or Majepains.

SECTION VII.

1 Ans cette derniere Section, qui fera la conclufion de noftre Liure, ie vous veux enfeigner la plus diuertiffante \& la plus agreable methode de confirs 
FR. A c ÇOIS.

les fleurs \& les fruirs de voltre Iardin, \&ren la pratique de laquelle je malleure que vous y prendrez plus de plainr que vousn'en poutuez fouhaitter; carles objets y font fi agreables que la veuë le trou. ue extremement recreée, en la diuerfité des couleurs que l'on y employe; \& auffi que l'Elprit fe plailt à inuenter mille gentilleffes (deriuées de tout ce que je vais efcrire) \& les ranger auec juftelfe pour fatisfaire ceus deuant qui ils cont prefentez.

Nous commencerons par les fleurs, \& dirons que l'on en fait des Conlerues de toutes fil l'on veut; mais je ne m arrefteray qu'à celles qui font les plus hautes en couleur, \& les plus agreables à manger, laiffant les autres que les Medecins ordonrént pour la fanté.

$L_{a}$ Violette comme celle qui vient la premiereau Printemps, fera mife en tefte de ce Chapitre, je ne m'alfujetriray pas pourtant a mettreles fleurs felon le rang que les faifons nous donnent, mais comme elles meviendront en la penfée.

Pour en faire de la Conferue vous éplucherez les fleurs ne prenant fimplement que ce qui elt Violet aux fucilles, \& les broyerezbien dans vn mortier de Marbre, ou de Pierre, tant que la pafte en 
264 LE IARDINIER

en foit fi deliée, quel'on n'y puiffe plus remarquer aucune forme de fueille.

Pendant que l'on broyera ces fleurs, wous mettrez du Sucre dans le poeflon pour en faire vise cuitte ou venuë, la plus grande fera de quatre liures au plus, \& la moindre d'vne liure, quoy que l'on en puiffe encore mettre moins, mais il y auroit trop de perte, à caufe du Sucre qui s'attache au poeflon : à quatre liures de Sucre, vous mettrez enuiror vne chopine, ou troisdemy feptiers d'eau, pour le faire fondre en l'arroufant par tout: vous mettrez voftre poeflon fur le feu de Charbon à demy embralé, afin que le Sucre fe fonde à loilir ; puis quand il bouillira vous l'efcumerez auec la gafche, \& le remuërez fouuent afin quil fe cuife également : vous donnerez le feu à voltre poeflon vn peu plus par le deuant que par derriere, afin que l'écume pouffée par le boüillon vers la queuë du Poeflon re puiffe plus facilement enleuer : \& apres qu'il fera bien écumé. vous mettrez le feu fous le milieu de voAtre poeflon.

Pour connoiftre quand voftre Sucre fera cuit en confiftence de Conferue, vous le pou: rez partrois façons: $\mathrm{L} a$ premie$r e$, quand en tournant \& meflant vofire

Sucre 


$$
\text { Fir a c çots. }
$$

Sucre de tous coftez, vois retirerez voAtre gafche fans l'égoutter que bien peu, \& la fecouiant comme fi vous vouliés pouffer vine balle-d'arriere-main auec la raquette vous verrés qu'il fe fera en filalfe volante. La feconde connoilfance eit quand ayant retourné voftre Sucre, vous laiffez égoutter voftre gafche, \& que dans les dernieres gouttes qui tombent, il refte à la gafche comme vn petit filet qui remonte fe retortillant en forme de queuë de Cochon; Et la troifiéme, quand apres auoir bouilly long-temps, vous voyez qu'il s'époifft, \& au licu qu'il faifoir fon bouillon dans le milieu, il fe fait de tous coftés, mais plus lentement? parlà vous ponu:z juger qu’il eft cuit. Vousle tirerez de deffus le feu, \& luy laifcrez paffer la plus grande chaleur: apres quoy rous prendrez voltre Pafte de Violettes, la mettrés dans le Sucreen motte, a vin des cuftez de voftre poeflon, \& la deftrempercz petit à petic auec la gafche : puis vous la menlerés par tout le poiflon, la tournant \& renuerfant uec le plus de promptitude que vous jourrés; \& ce jufques a ce que la chaleur n foit appaifée, ce que vous raconnoirés quand le boiiillon celfera, \& que oltre Sucre fera cn repos ayant diffipe 
266 LE IARDINTER

l'humidité qui eftoit aux Violettes.

Pour la doze, à quatre liures de Sucre il faudra enuiron gros comme le poing d'vn Enfant de dix ans, ou trois balles de tripot, de fleurs de Violettes pilées.

Ayant palle dans le poeflon fa plus grande chaleur, vous la drefferez ou en la verfant dans des tourtieres de papier bicn fechées pour eftre coupée en bifcuits: ou en plateaux que puiferez dans le pocflon auec la cueilliere d'argent, efremami \& prenant toûjours le delfus, qui fera comrne vre petite glace, \& verfant la cucillerée fur du papier bien $\int e c$, cela fera vn petit rond grand comme vne picce d'vn efcu: ou bicn en prenant fur 1a gafcie, \& auec le coufteaula couper par maccarons ; mais il faut qu'à cette derniere façon voltre Sucre foit prefque froid, autrement il couleroit de deffus la gafct:c; quand vous voudrez en faire des maccarons de toute la poeflonnée, il ne faudra prendre que par vn colté de voltre Pocflon, \&r non pas de tous coltés: à caufe que voftre Conferue fe refroidiroit trop, \& qu'il en demeureroit beaucoup au poeflon.

Pour retirer du poeflon toute voltr Conferue jufques à la derniere miette: ily faudra mettre trois ou quatre goutte: 


\section{Fra c ços s.}

deau \& en arrouler los bords 267
oftre Conferue; puis le preferter au feu par tous les coltez, \&rà mefure qu'il chauffera, déiacher voltre Conferuedu poeflon, l'amalfer en monceau auec la gafche, \& la tailler en maccarons comme la promiere tirée: Cette derniere Conlerue ne fera pas fi haute en fa couleur verdaffe que la premiere, à caufe de la craffe du Sucre qui s'attache au poeflon, \& anfi que toute cuuleur remile au feu diminuë beallcoup de fa beauté.

Vous noublierez pas à trauailler vos bifcuits dans vos tourtieres auant qu'ils foient froids, car ils fe romproient.

Vous leuerez vos. Canferues de d.ffus les papiers cuand elles auront encore vn peu de chaleur, fi vous n'cn voulés brifer beaucoup; \& fiellis font trop froides St que vous ayés peine à les leuer, vous chaufferés vnaix, \& polerés les papiers defius, celafera ieluër vos Conferues, \& elle ie leueront facilement.

Vous obferuerés trois chofes trés ne: celfaires en toutes les Conferues: La premiere, que vous foyezen lieu chaud, \& bienfermé, à caule que le froid vous empefche d'auoirle temps de verfer \& façonnervos Cenferues. La feconde, que *ostables loient propres, \& n'ayant au- 
268 LE IARDINIER

cune grailfe : car la Conferue dars fa chaleur eftant verfée, attireroit cette graifo fe àtrauers lé papier, ce quil l'empelcheroit de fe prendre. Et la troiliéme, que les papiers fur lefquels vous ta verfez \& dreliez, foient bien blancs; bien nets, \& bien fechez.

Imprimez vous bien dans l'efprit tout ce que ie vous viens de dire: car cela vous ferrira en toures les autres que $j$ e vous diray-cy apres.

Les fleurs de Badrfache, Bugloffe, Soulcy, Jalmin, \& les fueilles de Femoiiil vert, fe confifent de la mefmeme. thode que les Violertes.

Le Srrop violat eftant plutont vite boiffon qu'vn medicament: $j$ 'ay creuneceffaire de vous en donner la compolition, quoy qu'il femble que je le deurois plutoft auoir mis au rang des Confitures liquides qu'en cét endroit cy: neant moins parce qu'il ne fe confit point de Violetres ou liquide, \& que cér article vous euft -efté difficile à trouucr dans voftre Liure, je l'ay placé en ce lieu pour voltre plus grande commodité.

Il y a deux manieres de le façonner; L'vne en broyant dans le mortier de pier. e vne liure ou enuiron en fleur de Violettes preparés comme pour la Confes- 


\section{Frit A çOT 5.}

te, Bzles metrre dinis vn linge alfez fort pour refiiter à la preffe, afin d'exprimer tout le jus des Violettcs, de laquelleliure vous en tirerez plus d'vn quarteron, fi les Violettes font fraifches cueillies: pendant que l'on les pilera \& pailera, Pous ferez cuire vne liure de beau Sucre, jufques à la confiftenco de Conferue, ( ce qui fe reconnoittra par les trois indices que je vous viens d'enfeigner; ) eftant cuit vous le tirerez du feu, hiy laiffercz palfer fon bouillon, \& apres verferez petit à petit ce jus de Violettes dedans; remuant atiec la gafche par tout le poeffon, puis eftant vin peu froid, le mettrez dans. des bouteilles de verre que boucherez. bien'auec des tampons de licge, \& de la cire molle par deffus; celle dont je vous ay donné la compofition àla fin du premier Traitté y fera fort propre. 'L'autre maniere eft de broyer des fieurs, lcs metere dans vine eftamine, ¿u verfer par deflus da Sucrécuit vin peu moins qu'en. Conferte, \& ce ì plufieurs repri es; peirdant qu'vn verfera, vn autre froiffera les Violettes auec vne cueilliere de bois, pour en exprimer le jus que receurez dans quelque terrine verniffée, \& apres que tout fera palfe il faudra mettre l'Efta. mine dans la prelfe pour ne rien per. 
270 IE IARDINIER

dre: Vous lailferez bien refroidir le tout, puis le ferrerez dans des bouteilles comme le precedent.

On mettra fecher à l'efture le marc de ees Violettes pour ne rien laifler perdre, cela eft a fiez agreable à manger.

11 fe fait de parfaitement beau Syrop. violat par infufion, comme aufil de Roles pafles, defieurs de Pefcher, \& beaucoup d'autre encore: Mais comme cela eft du fait de la Medecine, je ne m'entremettray point d'en dire aucune chole, quoy qu'ils foient trés-faciles à faire.

La Rofe de Prouins fe confit en Conferue lesche \& liguide : la liquide fo fait en prenant des Rofes quand clles commenceni à s'épanoüir, \& leur ofter tout ie vert, \&t le jaune melme quieft ì chaque fueille, les bien broyer dansle mortier de pierre, y adjouftant la moitiéautant de Sucre commun en poudre, comme il y aurade pefanteur de fleurs toutes épluchées, \& les mettant dansle mortier petit à petit à mefure que l'on broyera; \& eftant bien brojée, vous la mettrez dans des pots de grets que vous n'cmplirez pas tous pleins, à caufe qu'elle fe fermentera (c'eft a dire qu'il fe fera comme vin leuain qui fera bouffir voftrc Conferue : Quand vous aurez mis vos pots 


\section{R A N çOrs:}

278

au Soleil, \& qu'ilsy auront efté par l'erpace de quinze jours durant : Vous les retirerez à l'ombre quatre ou cing jours, les remplirez de l'vn deux, \& les couurirèz bien auee du parchemin en double, que moiiillerez pour le faire mieux joindre au pot, lequel vous lierez d'vne ficelle.

Pour la Conferue de Rofes fecher de la maniere que l'on la fait à Prouins, il fatit prendre des Rofes toutes épanoilies, leur ofter tout le vert, \& le jaune entie-rement, comme pour les Conferues liquides, les mettre fecher dans des fachets de papier, \& eftans parfaitement feches les battre aut mortier de fonte, en l'enueloppant \& le pilon auffi auec vn fac de cuire ainfi que les Parfumeurg battent leurs poudres : eftant battue il la faudra paffer au tamis de foye delié, puis la bien enfermer dans des papiers \& boëttes, crainte ds l'éuent pour s'en feruir au befoin.

Quand vous voudrez faire cette $\mathbb{C}^{-1}$ ferue vous prendrcz quatre onces de voftre poudre, \& la mettrez dans in plat de fayence, vous efpreindrez des Citrons dans vin verre pour en tirer le jus, que laifferez repofer pour eftre plus clair \& plus beau, d'autant que la fece ou le plus 
272 IIIARDINIER

épois \& limoneux du Cirron defcendra au fond du verre: eftant clarifié, yous. en metrez parmy voftre poudre pour luy. faire venir fa bellecouleur en la deftrempant auec la gafche, \& la retournant bien de tous coltez: pendant ce tempslà, vous fercz cuire deux liures de Sucre jufques à la confiftence de Conferue forre, puis le tirerez du feu, luy laiferez: abatre fon boiillon, \& metrez cette pondre à vu descoftez de voltre poeflon, la defrempantpetit à petit, \& la meflant bi n atiec le fucre; voitre conferue fera firite.

Pour la drelfer par Maccarons, comme font celixde Prouins, vous en prendrés fur la Gafche, \&r auec le coufteat la taillerés \& drelferés fur des papiers fechés : fi elle fe refroidit par trop, \& qu'elle ne vous donne le loifir de la dreffer, vous mettrés voftre Poeflon fur vn fetit feu bien lent, pourueu qu'il l'efchauffe vn peu, \&z la rende maniable il fuffira : vous retirerés iufques à la derniere miette de Conferue en pratiquant ce que ie vous ay dit à l'article des Violettes, \& auffi pour la retirer de detfus les papiers \& la ferrer dans les boettes. Quelques vnsy adiou?ent vn peu d'efprit deVitriol blanc, pour releuer dauantage 
là couleur de $\quad 273$ fi fain a de Role; maiscelan'e?t pas fi fain, ny naturel, que quand l'on y employe le ius de Citrón pur.

V. us obferuerés de ne pas rendre voAtre Palte de Role trop liquide auant que la mettre dans yoftre fucre: car il y auroit par trop de defcuit; à caufe du jus de Citron qqui defcuit estremenent, \&: en grailfe le fucre plus que toute autre chole : il fuffira qua elle foit abbreuée pas tout, \& que vous la puiffiez mettre en plorte fans qu'elle coule.

Tant plus voftre Conferue ent fraifche faite; tant plus belle elle eft ; c'ett pourquoy les Apoticaires de Provins en ont toûjours de trés-belles, parce qualils en ont grand debit: Quoy que je vous en aye dit que la compolition pour deux liures, ce nieft pas que ccux de Prouing ren falfent des poeflées enticres de dix ou douze liures de Sucre; mais comme vous n'en auez pastant de beloin à la fois. nien voulant pas faire trafic, il fuffira - du peu, puis que vous aurez tôkjours de la poidre de Rofes en voltre logis, pours: la faire en toute faifon.

L'on en fait aufir de Rofes blanches: mais elles pallent fi-toll leur beauté, \&s: ontfi peu degoult de Rofeserd'adeur quer: je ne l'eftime pas beansoup. 
274 LE I A R D I N IER

La Conferue de Rofes qui fe fert ordinairement dans les bals \& feftins, ne fe fait pas fi forte de poudre de Rofes que celle de Prouins, cardemie once de poudie, fera plus que fuffifante à deux liures de Sucre, y mettant autant de jus de Citron, qu'elle en pourra porter pour la rendre liquid:, de telle façon pourtant qu'en penchant la vailfelle où vous l'aurez détrcmpée, elle ne coule point, puis quand voftre Sucre fera cuit en Conferue, vous le tirerez de deffus le feu, luy lailferez paffer fon boüillon, \& y mettrez voltre Palte, la meflant continuellement auec la gafche, jufques à ce que le Sucre foit appaifé du boiillon que le décuit luy aura fait éleuer : Ce qu'eftant fait, vousverferez voltre Conferue dans les Tourtieres de papier pour en faire des Bifcuits, lefqucls fe façonneront tous couuerts de petices bouteilles, auec vn. glaffis bien reluifant: \& fi vous en dreffez en platteaux ils fe feront auffi par deffus de la mefme façon : le refte qui demeurera dansle poeflon vous l'amalferez \& le leuerez auec la gafche pour le couper en Maccarons.

Noubliez pas à couper vos Bifcuits, ny à leuer vos Conferues pendant qu'il y a quelque chaleur pour les raifons que. 
je rous ay dites cy-deuant \& foit rcpeté pour la derniere fois : car je n'es parleray plus.

Vous f̧aurez que $f 1$ vous détrempiez voftre poudre de Rofe dans quelque vaifelle d'argent ou d'eftain, que cela luy donneroit vne teinture plus violette: par quoy fi d'vne mefme poudre vous en voulezfaire de deux couleurs, il n' ${ }^{2}$ aura qu'à en mettre dans de la fajence $8 z$ dans de l'eftain.

Ie vous diray encore que la grande beauté de toutes les Conferues ne dure que quatre ou cinq jours au plus en leur bel éclat: c'eft pourquoy. l'on n'en feta qu'au befoin.

La Conferue de flcur d'Orenge fe fait en hachant fes fueilles bicn deliés $\&$ les jettant promptement dans le jus de $\mathrm{Ci}$. tron, de crainte qu'clles ne noircifient: dans deux liures de Sucre, vous y pourrez mettre gros comme vin efteuf de fleurs; ayant égoutté le jus, afind'y auoir moins de décuit: \& vous en ferez la Conferue comme je viens d'enfeigner à celle de Rofe, pour feruir en compagnie.

L'on fait vne Conicrue rauiliante de Framboifes blanches \&z rouges, en les preflant lcgercment, \& cn tirant le jas. que mettrez dans quelque verre pour le

$$
\text { M. } 51 \text {. }
$$


276 LE I A R D IN I E R

laiffer rafieoir, \& que fa lye aille $\mathrm{u}$ fond’s puis voftre Sucre eftant cuit en Conferue \& tiré hors du feu, vous verferezce jis dedans, yen metrant ce que vous jugerez que le Sucre pourra porter de décuit, puis vous la drefferez dans les tourrieres, la taillercz \& ferrerez a l'ordinaire.

Du jus de Gadelles, de Grenade, \& de celuy $D^{\prime} e$ pine vinette fe fait de trés belle éx trés-agreable Conferue, en s'y gouuernant comme au Framboifes.

Si vous voulez ougmenter le gouft aux Conferues faites auecl'vn de ces jus rouges, vous les coulerczà trauters le papier brouilllars, puis vous les ferez bouillir pour les reduire, afin qu'il entre beaucoup dans le Sucre, fans que cela le décuile par trop.

Pourfaire la Conferue d'Orenges, vous prend́rez de la râpure du delfus des Orenges, celle que vous aurez tiré des confires au fec pourra feruir: $\delta c$ à mefure que vous la râperez, elle tomberadans l'eau tiede ou fraifche, de crainte qualle ne uoirciffe : vous la mettrez infufer fur le feu en grande eau pour la defamertumer, \& reiitererez plufieurs fois jufques à ce qu' $c 1$ le ait perdu entierement fon amertume, ce qu eftant fait vous lo mettrez dans vi linge, \& en fersz fortir toute l'ẹay eds 


\section{F R A अ}

tordant le linge, par aprés vous la mettrez dans vin plat d'argent, \& la fecherez en la maniant continuellement car elle s'attacheroit au plat, \& ce jufques à ce qu'elle foit prefque toute feche: cependant vous cuirez du Sucre julques à la confiftence de Conferue, puis jetterez dedans voltre poudre, \& remuërez bien de tous côtiz, afin gruelle prenne corps auec voltre Sucre, fi vous y voulez met. tre vn peu de jus de Citron d'Orenge, apres que voftre poudre aura efté bien meflée, cela luy donnera bon gouft: Vous pourrez mettre à deux liures de Sucre gros comme vne balle de poudre d'Orenge, \& da jus à difcretion, fe prenant garde de ne pas trop dicuire voltre Sucre : vous la verferez, drefferez \& taillerez, comme celle do fleur d'Orenge.

Celle de Citron fe fera tout de mefme que celle d'Orenge, râpant non feulement la peau jaune, mais encorela chair jufques aujus, \& l’ayant bien lauée \& délimonnée en plufieurs eaux chaudes, \& fechée, comme j'ay dit celle d'Oremge, vous la mettrez dans le Sucre, \& y adjouterez le jus de Citron pour la rendre plus hautes en faueur.

Jour faire le Bifcuit de Citron épois 
278 LE IARDIIIER

de trois doigts \& plus, vous cuirez voAre Sucre en confiftence de Conferue vn peli forte, puis jetcerez la poudre de Cirron preparée comme jay dit, \&̂ mêlerez vn peu de jus de Citron \& du blanc d'ceuffouetté auec des verges pour le faire leuer, cequ'ayant fait, vous verferez voftre Sucre fur du papier, tenant le poeflon deffus, afin que $\Gamma_{a}$ chaleur falfe leuer d'auantage voftre Conferue, puis eftant encore chaude, vous la couprrez par gros Biicuits qui feront tous pleins d'yeux pardedans, \& fort legers.

On fait auffi de la Conferue de jus de Citron fans y mettre de la Chair râpée, mais elle n'a que l'acidité, \& n'ett pas fi agreableque celle où vous ea aurez mis.

Les Piftaches feront pelées à l'eau chaude, puis pilées dans le mortier de pierre, les arroufant auec l'eau de fleurs d'Orenges, de crainte qu'elles ne rendent l'huile apres quoy vous les metrez dans voftre Su.re cuit en Conferue, \& deftrcmperez cette Pafte à vn colté de voltre poeflon, mellerez bien le tout, \& y adjoufterez quelque peu de Mufc, \& d'Ámbregi: broyez suec du Sucre bien delié \& pa! fé au Tamis, puis da dreflerez commi les autres: 


\section{FR A c C O I S:}

Il fe fait de la Conferue de toutes fortes de Fruits desja confits au fec, comme Abricots, Pefches, Cerifes, Orenges, Citrons, Efcorce de Citron vertes, \& autres, en les taillant en lardons ou perites billes femblables à des jettautidans le Sucre alors qu'il eft cuit jufques à Conferue vn peu lafche, puis meflant le tout pour en rendre la diuerfité plus agreable, il les faudra dreffer \& taillerainfi que $j$ 'ay dit aux autres.

Vous poutuez en Conferue contre-faire plufieurs chofes qui furprennent à l'abord, comme par exemple des tranches de Jambon, cela eft vn peu embarralfant à vne perfonne qui n'a pas accouftumé de tra uailler en conferues, à cau fe qu' il faut bien prendre fon temps pour entendre à tout, mais pourtant trés facile à qui y à vin peu de pratique.

Pour y paruenir, vous au rez des plats d'argent tous prefts, \& quand voftre Sucre fera cuit en Conferue un peu foible, ou lafche, vous en verferez vne partie dans vin de vos plats, \& le poudrcrez de chair de Citron, on de jus de Fram, boifes blanches, ou de fleurs d'rerenges, bref de tout ce qui fera blanc, pour reprefenter la graiffe \& le lard, \& ferez aien tourner cette Confeine par quel. 
2SO LE IAR DINIER

qu'vn pour incorporer le Sucre atiec le décılit : puis vous verferez vne autre partie de valtre Sucre dans vn autre de vos plats, \& y mettrez do la poudre de Rofes, jus de Framboiles, Grofeilles rou. ges, ou autre qui pourra reprefenter la chair de Jambon, ne menant pas voltre décuit par tout le plat, afin que reftans quelques endroits blancs quand yous tirerezvoltre Conferue du plat, cela imite mieux les grailfes qui entre lardent le Iambon, \& pour dernier, vous metrez auec le refte du Sucre qui fera dans voltre poellon, de la fueillede Fenouil vert, que ferez piler dans le mortier de pierre, fi c'ut en la faifon, ou en autre temps des Piftaches, pour reprefenter le Perill haché, \& herbes fines, que l'on met fur le lard da Iambon: Vous prendrez ces Conferues qui ferone encore chaudes \& poferez la rouge, \& vn peu de blanche fur vae table, les meflerez enfemble pour imiter les mufcles \& grailfes, qui entre-lardent 'e Tambon: puis apres metrez de la blanche par deffus pour contrefaire le lard, \& du vert pour le dernier qui reprefentera le Perfil, vous ap. puicrez \& battrez de la main à chaque lict que mettrez afin de les incorporer 8 joindre enfemble. Vos Conferues eftas 


\section{F R A N C O I 5.}

28.1

encore chaudes vous les couperez en forme de tranches de Iambon, \& les drefferez fur desaffiettes, mettant yne ou deux fueilles de Laurier par deflus pour ayder à la tromperie.

Si vous en vouliez faire vne quantité, vous pourriez vous feruir de trois poeflons, au licu d'écuelles d'argent.

Pour contrefaire des Ceruelats, prenez le refte du rouge qui fera dans vos écuelles, \& y mettrez deux ou trois gouttes d'eau claire, ou de fleurs d'Orenges, menlez y de la poudre de Canelle battuë, \& palié bien fine pour embrunir voftre coulcur \& ramalfez les teftes de voftre Iambon, (oftant le vert), mettrez le tout dans le poeflon, aucc un pcu d'eau que vous verferezn'en prenant que dans vine cucilliere d'argent, peur d'en trop mettre, mais feulement pour décuire vn peu le Sucre: Vous mettrez le pocflon fur vn petit feu pour échauffer ces Conferues \& les incorporer enfemble en les meflant auec la gafche, \& qu'en les tirant du poeflon elles foicnt faciles à manier \& former auec lesmains en rond comme des Saucifons; pendant que le Sucre fera encore chand rous les trancherez par roëles, ou en long, \& les dreflerés fur des affiettes thinfi que les Sauciffons naturels. 
282 LE I A R D N TE R

Vous pourrez encore imiter mille ger: tillelles auec le fucre \& toutes ces couleurs, en faifant des paftes que vous mouflerez \& façonnerez à l'oifir d'autant qu'elles n'entrent point fur le feu.

Par exemple, pour contrefaire des $R u$. bans d'Angleterre de plufieurs couleurs Vous qrendrezdu fucre palfé bien delis au tamis de foye, \& pour le rendre encore plus fin que celuy qui ent pilé au Morticr, c'eft qu'il le faut broyer à fer fur le Marbreainfique les peinftres fon leurs couleurs : eftant pallè vous cn met trez fur vne tablebien nerte, y mélcre: vn peu de gomme agragant détrempé auccl'eau defleurs d'Orengesou de Ro fes, \& ferez vne pafte quilie puilic eften drefous le Roufleau: Vous diuiferez cer te paitecn autant de morceaux que vou voudrez qu'il y ait de couleurs, lefquel les vous menterez auec voftre fucre cha cune à part, les eftendrez auec le Roul leau les. plus minces \& tanues que vou pourrez: Vuus trancherez ces paftes $p a$ petites Lanieres bicn.delieés que ioir drez proprement\& promptement les vns anx autres, afin que l'humidité qui e dans le corps de la pafte foude ces Lanis res, que diuerfifierez pour imiter lcsk, bans: en fuitte de quoy, vous pallerez 1 . 


\section{FRA N ÇOI S.}

gerement le Roufleau par deffus pour les mieux ioindre, puis vous les fermerez en noeuds ou galans, mettant des Cartes pliécs en rond pour fouftenir les replis iufques à ce qu'ils foient fecs, ce qui fe firaen moins de deux heures.

Vous contreferezauffiles Chiffres, \& Armes blafonnées de qui vous voudrez, taillant cette pafte auec le coufteau, ou auec des moufles de fer blanc qu'aurez fait faire exprés, \& imiter toutes fortes de marqueterie que voftre efprit vous fuggerera.

Si vous voulez rendre ces figures enFore plus recreatilues:eftans parfaitement Eeches, vous les glacercz de fucre delié A:frempé auec caude fleurs d'Orenges, $x$ les mettru $z$ dans le four; puis les reirercz \& glacerez do lautre cofté; les. emettant derechef au four pour en feher la glace: parainfi on ne verra point a figure qui fera enfermée entre ces deux lacis, à caufe de la blancheur, fice n'eft In l'cxpofant au iour \& regardant à traers; alors on reconnoiftra ce que ceft, \& II admirera voftre induftrie.

Auec cette mefme pafte vous ferezdes blettes $\&$ mufcadins tels que vous vourez y meflant le Mufc, l'Ambre, la poure d'Iris, la Canelle, le Girofle, \&e 
284 IF IA $\mathrm{T}$ D I $\mathrm{X}$ I $\mathrm{E}$

route autre chofe qui peut feruir a la rea creation du gouft, on à fortifier l'eftomach ; mefme aufi des purgatifs, \& preferuatifs de toutes les fortes que la Medecine enleigne.

Pour feruir des Mulcadins fur table, l'on les met d'or inaire dans de petites tourtieres ou abbaiffes de la mefme pant? (mais fans Mulc pour éuiter à la def. pence ) ces petites Abbailfes fe font auec des Moufle da fer blanc qui feront de telles figures que vous voudrés, ou rondes, ou en Triangle, ou à Pans, ou en Eltoille, bref felon voitre fantaifie: dans lefquelles vous mettrés de voltre pafte qui aura efté citenduë aucc le Roufleau, $\&$ auant que de la metrre vous poudrerés voltre Tourtiere de fucre crainte qu'elle ne s'atrache: vous rognerés anec le coufteau le furplus de la figure de la tourtiere, \& laillerés fécher ces Abbaitles dans leurs moules environdeux heures: puis les retirés, \& porerés en quelqui lieu oì elles puilfent saeheuer de bin fecher.

Dans ces mefmes petites tourtierc quand elles feront bien feches, l'on dielfera des Confitures liquides pour e: mettre de plufieurs fortes liar vne mefo Aflicte. 


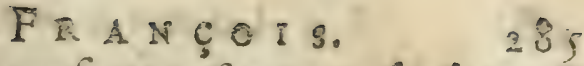

De cette mefne pafte vous ferés auffir des Bifcuits, \& pains de : itron, $y$ adioultan de la ralpure quaurés fait parboüillir pour ére plus a greable, \& l'ayant mené anee vinte lecrc \& Gomme : vous tirerés \& applatires la pafte fous le Roufleau, mettanudilfous \& deffus du fucre en poudre (de crainte qu'elle ne s'attache à la 'able ou au Rouflcau) fans la replier pourmnt, afin qu'e!le garde vne humidité dans lo milieu qui la falfe leuer, quand yous la mictrésau four, quil lera vin quart d'heure apres que vous l'aurés coupée en Biĺcuirs ou peits Pains, à caufe qu'elle fecheroit trop \& ne leueroit pas.

Si vous ne voulés vous feruir de Gomme Agragant, vin peu de blanc-d'ceuf fuetté y pourra fupplier.

Vous en ferés de tous Goults \& de toues couleurs, le Tourne ful (quoy que ort peu agreable: s'employera au deffaut les Violettes, Bourraches, \& autres coucurs femblables: fir vous y mettés du ius le Citron il luy changera fa couleur.

On trempe par fois dans ces couleurs es Cerifes, des Framboiles, \& des Gaelles, puis on les rounte dedans du fu- bien fin en leur lairlant prendre ce a'lles peunent, les mettant feiches 
$286 \quad$ LE I A D I N IE R

deuant le feu pour alfermir le fucre : Si vous meflez auscros couleursvn peud'eau deblane d'cufou de gomme, cela les rendra fermes pardeffus comme les dragées.

Le Feuoiiil vert grainé en branche \& autres fleurs, feront plongez dans le fucre cuit en Conferue, puis poudrés de fucre fin on les mettra fecher deuant le feu.

Au Fenoiiil vert, on y larde des Curedens comme l'on feroit des Gluaux à vine pippée, cela fert de dragée, \& pour fe netroyer la bouche.

Les Maflepans fe font d'Amandes dou. ces, d'Auclines, de Piftaches, de Pignons, d'Amandes ameres, de noyaux d'Abricots \& Pefches, melmement de Semences froides mondées fi vous y voulez faire la defpence: \&r ils fe trauaillent tous de la melme façon.

Pour le faire excellent, il faut prendre vne liure d'Amandes, que vous pelerez les mettant dans l'eau chaude, puis dans la fraich à mefure que vous les dé-d pouillerez de leur peau, car cela lcst t: blanchic de beau coup : vous les retircrea de l'eau, les effuyerezou feichcrez dan: du linge, \& les mettrez dans le Mortie! c de Marbre ou Pierre, \& les pilcrez tan' que l'on ne puide plus remarquez aticus 
FRA NÇOIs. $\quad 287$ se partie de l'Amande pour petite qu'el. le foit : en les pilant vous les arrouferez auec de bonne eau de flurs d'Orenges, ou de Roles bien rectifiée, \& purgé de fon Phlegme pardintilations; ou fimplement repolée de long.temps, car il s'y fait vne vaze au fonds dela boutcill qui cat le plus vifquemx \& Craífe de toute l'esu Role, \& faudra paffer ce que vous cu au. rez à faire à traucrs vn linge de crainte que ce Phlegme ne fo mole: ce que l'on y met des eaux de fenteur n'elt pas tant pour l'odeur gue pour empefcher que les Amandesen les pilant ne rendent l'Huile, car le araflepan feroit defagreable: vous y meflerez rne liure de beau fucre palfédelié comme cciuy d s Mulcadins \& en ferez vn corps en confilterce de buuillie bien efpoitle: vous patrerez voftre pafte d traucrs le fas ou l'Eliamine, afin que s'il y anoit quelques morceaux d amandesquine fulient pas allez pilezon les rebroyaf, \& que lion ne trouse rien de delagreabledansle malfepan: Vous mettrez cette palte dans le poeflon, \& la cuirez à petit feu comme les paftes de fruits fecs, ( $c$ eft à dire) iufques à ce que la pafte quitte le Poeflon, en retournant toújours de peur qu'elle ne bruhle: yous engoufterez fi elles tient de la cuifo 


\section{LE I A R I I I E R}

fon auant que de la tirer du feu = puis eftart cuite la verferez dans quel que vaiffelle dé Fayence qu'aurez poiidrez de fucre afin que le Maflepain ne s'y attache point, \&eftant prefque refroidie, vous Ia metrez fur yoftre table, la manierez \& tirerez fous le Roufleau comme de la pafte, \& la façonnerez en grandes Tardes rondes ou bien en petices que vous arrondirez; lescoupart auec le haut du Caliced'vn Verreaboire, en l'appayant furla pate, qui tera de l'efpoilfeur que vouder que bicni vos tartes: vous en ferez de quarrées, cn l'Jange, cn Cocur, en Trrangle, \& dz touse autr: figure que vous voudrez. les pinçrat par les bords pour y fairele petit dencelage qui s'y pratique ordinairemant : cftans façonuécs vous les mettrez cuiredans le four moyénement chaud, pour leur domer vne pezite couleur roulfe par les bords, \& ertremitez, puis les retirerez, les laillerez refroidir, se les glacerez auec fucre fin \& eau de rofe ou de fleurs d'Orenges: Il faut que cette glace foit efpoilfe comme du Miel blanc \& que l'on l'eftende auce le coufteau, apres quoy vous remetrez vos tartes au four pour cuire cette glace : \& quand clle aura bouffy rous les reirerez, les laillerez refroit- 


\section{dir, sefuretez en licat foc.}

$$
\text { Fraw corso } 28
$$

Aux grandescaries apres que vous leur aurés donné cunleur, yous les glacorés, \& piqueres dellis des Piftaches, des C 0 corces de Citron de l'Orengeat, du Citronnat, ou du Canelas en dragées : puia vous les remettrés au four pour fecher la glace, \& les retirerés quand elle aura bouffy.

Sivous voulés les embellir \&z enrichir dans le milieu de quelque petis Rocher de Confitures diuerfes, vous en mertrés de telle forte que voudrés, puifque vous aués la methode de les mettre er conferue.

Vous ferés auffi aue voltre malfepan de longstriangles pour de trois en dreffer vine Piramide fur vn plat que vous accompagnerés de petites tartes tout autous on de Mallepars filé (dont ie vous vais enfeignercy-apres la maniere de le faire, ) \&z adiufterés fe tout felon que vous in. uenterés par voltre caprice.

Le mallepan de Piftaches (qui fe fera comme celuy d'amandes douces) vous lepourrés déguifer en fonds d Artichaux, en Afperges, mettant vin peu de blancaus gros bout pour mielix tromper, en pois fanscoffes, \&z autres choles fembiables, ay aist des moutles de bois ou de plomb 
290. L E I A R D I N I ER

qui en fallent la figure, bref vous vous. diuertires dans ces gentilleffes autant que volte curiofité vous y portera, \& la defpence que vous woudrés faire y pourra fournir.

Auec du mallevan d'Auelines, vous pourrés faire des petits boutons que cuirésaufour, leur faifant prendre vn peu de conleur pour contrefaire les Auelines, \& les feruirés dans vn plat comme en $\mathrm{Ca}$. refme on fert les naturelles.

Si vous ne voulez mettre tout voftre Malfepancu tartes, vousle prendrez aucc la gafche dans le poëflon tout fortant du feu, \& auec le coufteau en drelfercz: dics Aaccarons comme ceux de conlerue, que metrez au four pour prendre collleur.

Les Paticicrs dans leur malTepan \& Maccarons y meflent vn peu de fleur de farine de pur froment bien fine, \& de l'eau de blanc-d'Oeuf dans la glace c'eft ce qui les rend pafles: pour prendre de cette cau il faut foetter des blancs d'cufs, \& les lailfer repofer: fous la moulfe, il fe trounera de l'eau auec laquelle vous deftremperezvoltre glace, \& voltre pafte pour les maccarons.

Pour faire le mallepan fillé, il faut anirvne petice pallette de bois, us va 


\section{F R A ÇOIs,}

sond de boëtse, qui aura des cordotis pour l'attacher à la main, \& roufler deffous de la pafte de maffepan, en poudrant foutent voltre table, de crainte qu'il ne $s^{\prime} y$ attache : il fe tirera long \& efgal en groffeur, duquel vous ferez des Lacs d'ameur, des Tortis, des Chapeaux, des baftons rompus, des Chiffres, \& au tres figures telles que voudrez: vous les mettrez au four pour prendre couleur, $\&$ les ferrerez pour enioliuer \& adiufer vos autres malfepans.

De voftre mefme pafte de maffepans, vous en pourrez faire des Tourtieres ois Abbailfes grandes $\&$ petites qu' emplirez de confitures, au lieu de celles de pur fucre que iay dit cy-deuast.

L'on tire auffi des matrepans en bande, c'eft à dire que l'on fait des Compartimens, \& Armoires, lefquelles on remplit de Confitures liquides de la couleur des Blazons, comme pour reprefenter l'Or on mettra des Abricots, ou de la marmelade d'Abricots, \& Pommes : le Sinople, du Verius, des Amandes, \& des Ab cots Verts; Gueufle, des Cerifes, Framboifes, \& Grofeilles Rouges: ainfi des autres, approchant le plus que l'on pourra de ce que l'on veut imiter. 
202 LEIARDINIER

S. Tous rianz vn four de Nalfonnerie che vous, f'on en fait de Cuinre rouge dont vous pourrez vous feruir aued plus de commodité que des ordinaires, en pofant doucement fur des papieres vos Maffepans \& glacis, \& mettant du f i de charbon delfus, \& deffous, cela fatisfora à vos intentions: il fera de befoin d'y regarder foument, afin de ne 2unner plus de cuiffon à vos maffepans, Pains de Citron, \& Glacis, que ce gue rous voudrez qu'ils en prenient.

\section{FIN.}


10.30 log/w799. coldi onde

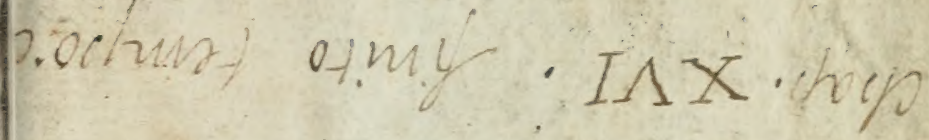

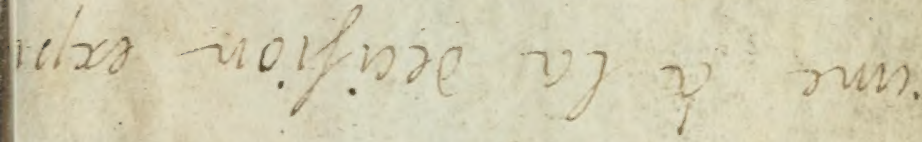

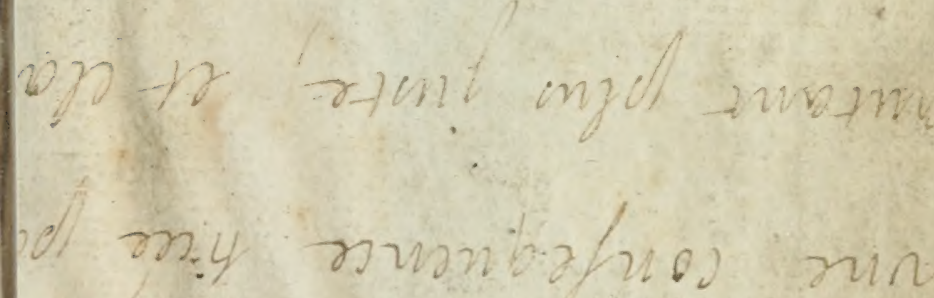
(a) -2 y)

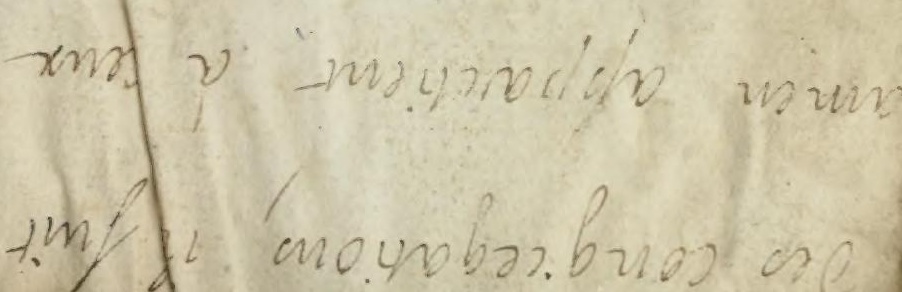




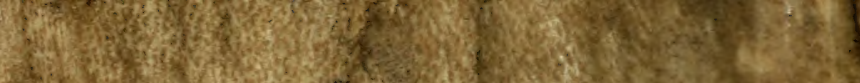

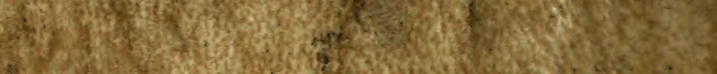
W. 20 Q

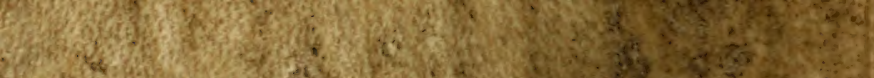

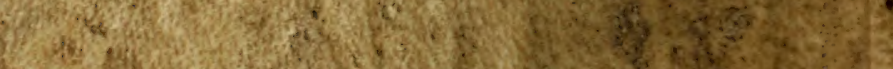

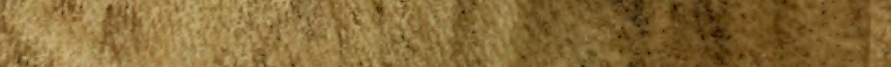

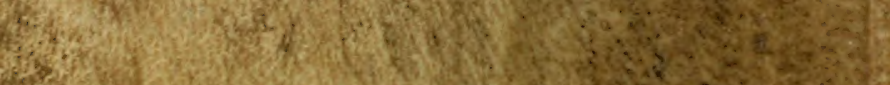

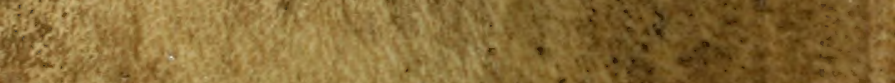

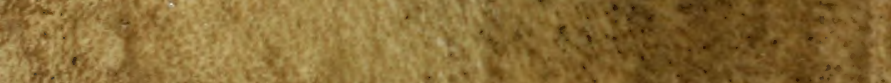

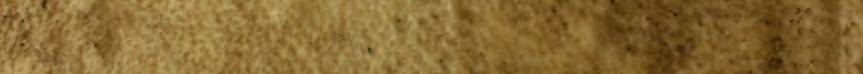

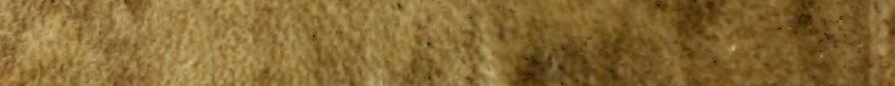

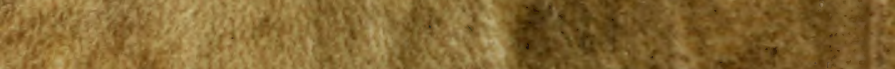

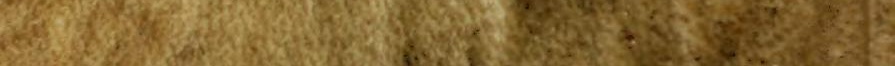
W. 3 (2)

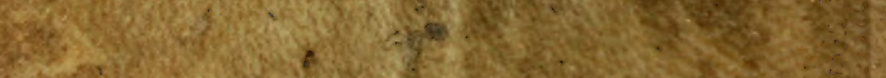

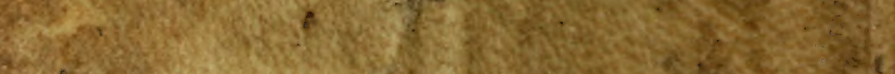
of

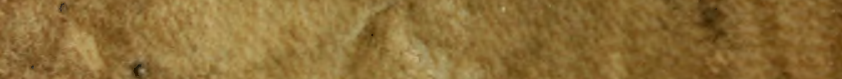

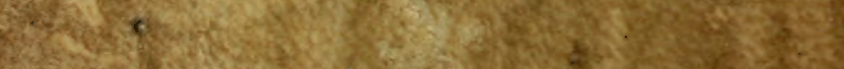
(1)

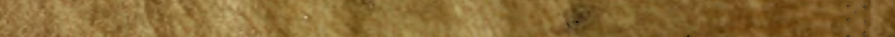
tong (6) Nit.

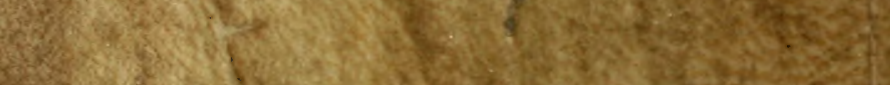

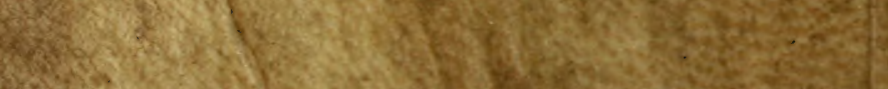

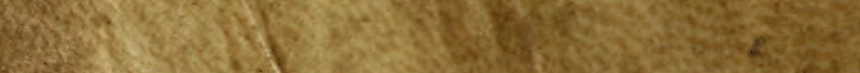

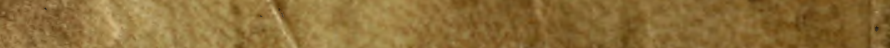

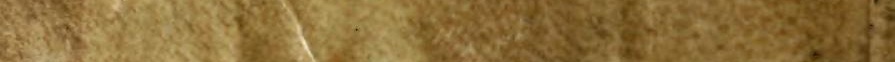
3. (2) s. 